\title{
THE SQUARE OF A HAMILTON CYCLE IN RANDOMLY PERTURBED GRAPHS
}

\author{
JULIA BÖTTCHER* ${ }^{*}$ OLAF PARCZYK ${ }^{\dagger}$, AMEDEO SGUEGLIA* ${ }^{*}$ AND JOZEF SKOKAN*,§
}

\begin{abstract}
We investigate the appearance of the square of a Hamilton cycle in the model of randomly perturbed graphs, which is, for a given $\alpha \in(0,1)$, the union of any $n$-vertex graph with minimum degree $\alpha n$ and the binomial random graph $G(n, p)$. This is known when $\alpha>1 / 2$, and we determine the exact perturbed threshold probability in all the remaining cases, i.e., for each $\alpha \leq 1 / 2$. We demonstrate that, as $\alpha$ ranges over the interval $(0,1)$, the threshold performs a countably infinite number of 'jumps'. Our result has implications on the perturbed threshold for 2-universality, where we also fully address all open cases.
\end{abstract}

\section{INTRODUCTION}

In extremal graph theory, the square of a Hamilton cycle often serves as a good concrete but reasonably complex special case when results about the appearance of a more general class of structures are still out of reach. Here, a Hamilton cycle is a cycle through all the vertices of a graph, and the square of a graph $H$ is obtained from $H$ by adding edges between all vertices of distance two in $H$.

For example, a well-known conjecture by Pósa from the 1960s (see [13]) states that any $n$-vertex graph $G$ of minimum degree at least $2 n / 3$ contains the square of a Hamilton cycle. This was solved in the 1990s for large $n$ by Komlós, Sarközy, and Szemerédi [22], demonstrating the power of the then new and by now celebrated Blow-Up Lemma. Only more than 10 years later the analogous problem was settled for a more general class of spanning subgraphs [10], using the result for squares of Hamilton cycles as a fundamental stepping stone.

In random graphs, on the other hand, the threshold for the containment of the square of a Hamilton cycle was determined only very recently and, surprisingly, this proved to be much harder than the corresponding problem for higher powers of Hamilton cycles (see also Section 1.1). Here, the model of random graphs considered is the binomial random graph $G(n, p)$, which is a graph on $n$ vertices in which each pair forms an edge with probability $p$ independently of the other pairs. By a simple first moment calculation the threshold for the appearance of a copy of the square of a Hamilton cycle is at least $n^{-1 / 2}$. Resolving a conjecture of Kühn and Osthus [25] and improving on results of Nenadov and Škorić [27] and of Fischer, Škorić, Steger, and Trujić [15], it was only recently proved that

\footnotetext{
* Department of Mathematics, London School of Economics, London, WC2A 2AE, UK. E-mail: $\{j$.boettcher|a.sgueglialj.skokan\}@lse.ac.uk.

$\S$ Department of Mathematics, University of Illinois at Urbana-Champaign, Urbana, IL 61801, USA.

† Institute of Mathematics, Freie Universität Berlin, Arnimallee 3, 14195 Berlin, Germany. OP is supported by the Deutsche Forschungsgemeinschaft (DFG, German Research Foundation) under Germany's Excellence Strategy - The Berlin Mathematics Research Center MATH+ (EXC-2046/1, project ID: 390685689). Part of this research was conducted while OP was a visiting fellow at the London School of Economics and supported by the Deutsche Forschungsgemeinschaft (DFG, Grant PA 3513/11).

E-mail: parczyk@mi.fu-berlin.de.
} 
$n^{-1 / 2}$ is indeed the threshold by Kahn, Narayanan, and Park [21], using tools from the pioneering work of Frankston, Kahn, Narayanan and Park [16] on fractional expectationthresholds.

In this paper our focus is on a combination of these two themes. We consider the question when the square of a Hamilton cycle appears in the randomly perturbed graph model and completely settle this. The randomly perturbed graph $G_{\alpha} \cup G(n, p)$, a model introduced by Bohman, Frieze, and Martin [5], is a graph obtained by taking a deterministic graph $G_{\alpha}$ on $n$ vertices with minimum degree at least $\alpha n$ and adding the edges of a random graph $G(n, p)$ on the same vertex set. In this model, which received a large amount of attention recently (see also Section 1.1), one is interested in determining the behaviour of the threshold for a given graph property in dependence of $\alpha$. More precisely, we say that $\hat{p}_{\alpha}(n)$ is the (sharp) perturbed threshold for a property $\mathcal{P}$ and a fixed $\alpha \in[0,1)$ if there are constants $C>c>0$ such that for any $p \geq C \hat{p}_{\alpha}$ and for any sequence of graphs $\left(G_{\alpha, n}\right)_{n \in \mathbb{N}}$ with $\delta\left(G_{\alpha, n}\right) \geq \alpha n$ we have $\lim _{n \rightarrow \infty} \mathbb{P}\left(G_{\alpha, n} \cup G(n, p) \in \mathcal{P}\right)=1$, and for any $p \leq c \hat{p}_{\alpha}$ there exists a sequence of graphs $\left(G_{\alpha, n}\right)_{n \in \mathbb{N}}$ with $\delta\left(G_{\alpha, n}\right) \geq \alpha n$ such that $\lim _{n \rightarrow \infty} \mathbb{P}\left(G_{\alpha, n} \cup G(n, p) \in \mathcal{P}\right)=0$.

For $\alpha=0$ the perturbed threshold is simply the usual threshold for purely random graphs, and the perturbed threshold is 0 for any $\alpha$ such that all graphs with minimum degree $\alpha n$ are in $\mathcal{P}$. In this sense, randomly perturbed graphs interpolate between questions from extremal graph theory and questions concerning random graphs. For small $\hat{p}_{\alpha}$ (and hence relatively large $\alpha$ ) there are some analogies to the concept of smoothed analysis in the theory of algorithms (see, e.g., [32]), and for small $\alpha$ one asks how much random graph theory results are influenced by the fact that in a random graph there may be vertices with relatively few neighbours. But in general one would like to determine the evolution of the perturbed threshold for the whole range of $\alpha$, which has so far only been achieved for very few properties.

This paper contributes to this line of research by determining the perturbed threshold for the containment of squares of Hamilton cycles for $0<\alpha \leq \frac{1}{2}$, which answers a question of Antoniuk, Dudek, Reiher, Ruciński and Schacht [3] in a strong from. In the range $\alpha \in\left(\frac{1}{2}, \frac{2}{3}\right)$ the perturbed threshold for squares of Hamilton cycles was determined by Dudek, Reiher, Ruciński, and Schacht [12], as we discuss in more detail in Section 1.1. The case $\alpha=0$, on the other hand, is the purely random graph case addressed in [21], and the range $\alpha \geq \frac{2}{3}$ is the purely extremal scenario addressed in [22]. Therefore, our result completely settles the question of determining the perturbed threshold for the square of a Hamilton cycle for the whole range of $\alpha$.

Theorem 1.1 (Square of Hamilton cycle). The perturbed threshold $\hat{p}_{\alpha}(n)$ for the containment of the square of a Hamilton cycle is

$$
\hat{p}_{\alpha}(n)= \begin{cases}0 & \text { if } \alpha \geq \frac{2}{3}, \\ n^{-1} & \text { if } \alpha \in\left[\frac{1}{2}, \frac{2}{3}\right), \\ n^{-(k-1) /(2 k-3)} & \text { if } \alpha \in\left(\frac{1}{k+1}, \frac{1}{k}\right) \text { for } k \geq 2, \\ n^{-(k-1) /(2 k-3)}(\log n)^{1 /(2 k-3)} & \text { if } \alpha=\frac{1}{k+1} \text { for } k \geq 2, \\ n^{-1 / 2} & \text { if } \alpha=0 .\end{cases}
$$

In other words, as long as $\alpha \in\left(\frac{1}{3}, \frac{2}{3}\right)$ it suffices to add a linear number of random edges to the deterministic graph $G_{\alpha}$ for enforcing the square of a Hamilton cycle, and for $\alpha \leq \frac{1}{3}$ the perturbed threshold $\hat{p}_{\alpha}(n)$ exhibits 'jumps' at $\alpha=\frac{1}{k+1}$ for each integer $k \geq 2$, where an extra log-factor is needed for $\alpha$ precisely equal to $\frac{1}{k+1}$. A similar 'jumping' phenomenon for the perturbed threshold has been already observed for other subgraph containment 
problems: for $K_{r}$-factors in [17] and for $C_{\ell^{-}}$factors in [8, 9]. However, Theorem 1.1 is to the best of our knowledge the first result exhibiting a countably infinite number of 'jumps'. Moreover, for $\alpha$ tending to zero, the threshold $\hat{p}_{\alpha}(n)$ tends to $n^{-1 / 2}$, which is precisely the threshold for the square of a Hamilton cycle in $G(n, p)$ alone as discussed above.

Since the square of a Hamilton cycle on $n$ vertices contains each $n$-vertex graph with maximum degree two as a subgraph, as a corollary to Theorem 1.1 we also get the following result, establishing the perturbed threshold for 2-universality for all $\alpha$. Here, we say that a graph is $r$-universal if it contains all graphs of maximum degree at most $r$.

Theorem 1.2 (2-universality). The perturbed threshold $\hat{p}_{\alpha}(n)$ for 2-universality is

$$
\hat{p}_{\alpha}(n)= \begin{cases}0 & \text { if } \alpha \geq \frac{2}{3}, \\ n^{-1} & \text { if } \alpha \in\left(\frac{1}{3}, \frac{2}{3}\right), \\ n^{-1} \log n & \text { if } \alpha=\frac{1}{3}, \\ n^{-2 / 3} & \text { if } \alpha \in\left(0, \frac{1}{3}\right), \\ n^{-2 / 3}(\log n)^{1 / 3} & \text { if } \alpha=0 .\end{cases}
$$

The range $\frac{1}{3} \leq \alpha<\frac{2}{3}$ is a corollary of our Theorem 1.1, while the remaining cases follow from known results. For $\alpha=0$, this is due to Ferber, Kronenberg, and Luh [14], for $\alpha \geq \frac{2}{3}$ to Aigner and Brandt [1], and for $\alpha \in\left(0, \frac{1}{3}\right)$ to [28]. The result in the range $\alpha \in\left[\frac{1}{3}, \frac{2}{3}\right)$ significantly strengthens one of our results from [7], where the same result was established for the containment of a triangle factor only.

Observe that the perturbed threshold for the containment of the square of a Hamilton cycle and the perturbed threshold for 2-universality differ for $\alpha<\frac{1}{3}$. This is due to the fact that in this regime the structure of the deterministic graph $G_{\alpha}$ may force us to find many copies of the square of a short path in $G(n, p)$ if we want to find the square of a Hamilton cycle in $G_{\alpha} \cup G(n, p)$ (see Section 3.2 for more details).

1.1. Related work. As indicated before, there has recently been a wealth of results on properties of randomly perturbed graphs. Let us close our introduction by briefly reviewing those concerning the containment of $r$-th powers of Hamilton cycles. Analogously to the square, the $r$-th power of a graph $H$ is obtained from $H$ by adding edges between all vertices of distance at most $r$ in $H$.

We start with the case $r=1$. Here, the case $\alpha \geq \frac{1}{2}$ is the classical Theorem of Dirac [11], which asserts that each $n$-vertex graph with minimum degree at least $\frac{1}{2} n$ has a Hamilton cycle, hence no random edges are required. The case $\alpha=0$ is treated by a famous result of Pósa [29], which shows that in $G(n, p)$ the threshold for the containment of a Hamilton cycle is $n^{-1} \log n$. For $\alpha$ between these two extremes, Bohman, Frieze, and Martin [5] determined the perturbed threshold. They proved that for any $\alpha \in\left(0, \frac{1}{2}\right)$, the randomly perturbed graph $G_{\alpha} \cup G(n, p)$ has a Hamilton cycle for $p \geq C / n$ with $C$ sufficiently large, and that this is optimal because for making the complete bipartite graph $K_{\alpha n,(1-\alpha) n}$ Hamiltonian we need a linear number of edges.

Turning to $r \geq 2$, proving a conjecture of Seymour [31] for large $n$, Komlós, Sarközy, and Szemerédi [23] showed that any large $n$-vertex graph $G$ with minimum degree $\delta(G) \geq$ $\frac{r}{r+1} n$ contains the $r$-th power of a Hamilton cycle, thus establishing that the perturbed threshold is 0 for $\alpha \geq \frac{r}{r+1}$. In $G(n, p)$ alone, i.e. when $\alpha=0$, it follows from a much more general result of Riordan [30] that $n^{-1 / r}$ is the threshold for the containment of th $r$-th power of a Hamilton cycle. The perturbed threshold behaves differently for any $\alpha>0$, as was shown in [6], where it is proved that in $G_{\alpha} \cup G(n, p)$ for any $\alpha \in(0,1)$ there 
exists $\eta>0$ such that the perturbed threshold for the containment of the $r$-th power of a Hamilton cycle is at most $n^{-1 / r-\eta}$, and it was asked what the optimal $\eta$ here is. In this paper we answer this question in the case $r=2$.

More is known for $\alpha \geq \frac{r}{r+1}$. In this regime, where $G_{\alpha}$ alone contains the $r$-th power of a Hamilton cycle, Dudek, Reiher, Ruciński, and Schacht [12] showed that adding a linear number of random edges suffices to enforce an $(r+1)$-st power of a Hamilton cycle. This was improved by Nenadov and Trujić [26] who showed that one can indeed enforce the $(2 r+1)$-st power of a Hamilton cycle with these parameters. When $\alpha>\frac{1}{2}$, even higher powers of Hamilton cycles have been studied by Antoniuk, Dudek, Reiher, Ruciński, and Schacht [3], who proved that in many cases the perturbed threshold is guided by the largest clique required from $G(n, p)$.

For certain values of $\alpha$ and $r \geq 3$, the perturbed threshold is not yet precisely known for $K_{r+1}$-factors (see, e.g., [7] for more details). This suggests that determining the behaviour of the perturbed threshold in the entire range of $\alpha$ for the $r$-th power of a Hamilton cycle for $r>2$ may be challenging. We discuss this further for $r=3$ in Section 8 .

Organisation. The rest of the paper is organised as follows. In the next section we introduce some fundamental tools, which we will apply in our proofs. Then in Section 3 we discuss a more general stability version of our main result and provide an overview of our proofs, together with the lower bound constructions and some auxiliary lemmas. In Section 4 and 5, we prove the extremal case and the non-extremal case of our main result, respectively. In Section 6 we prove a technical lemma that is used in Section 7 to prove the auxiliary lemmas. We then finish with some concluding remarks and open problems in Section 8. A few standard proofs are postponed to Appendix A.

Notation. For numbers $a, b, c$, we write $a=b \pm c$ for $b-c \leq a \leq b+c$. Moreover, for nonnegative $a, b$ we write $0<a<b$, when we require $a \leq f(b)$ for some function $f: \mathbb{R}_{>0} \mapsto \mathbb{R}_{>0}$. We will only use this to improve readability and in addition to the precise dependencies of the constants.

We use standard graph theory notation. For a graph $G$ on vertex set $V$ and two disjoint sets $A, B \subseteq V$, we let $G[A]$ be the subgraph of $G$ induced by $A, G[A, B]$ be the bipartite subgraph of $G$ induced by sets $A$ and $B, e(A)$ be the number of edges with both endpoints in $A$ and $e(A, B)$ be the number of edges with one endpoint in $A$ and the other one in $B$. More generally, given pairwise-disjoint sets $U_{1}, \ldots, U_{h} \subseteq V$, we let $G\left[U_{1}, \ldots, U_{h}\right]$ be the induced $h$-partite subgraph $\bigcup_{1 \leq i<j \leq h} G\left[U_{i}, U_{j}\right]$ of $G$. Given an integer $r \geq 1$, we denote by $G^{r}$ the $r$-power of $G$, i.e. the graph obtained from $G$ by adding edges between all vertices of distance at most $r$ in $G$. We denote the path on $k$ vertices and a Hamilton cycle by $P_{k}$ and $C_{n}$, respectively. Given a subset $W \subseteq V(G)$ and $v \in V(G) \backslash W$, the notation $N_{G}(v, W)$ stands for the neighbourhood of $v$ in $W$ in the graph $G$, and we denote its size by $\operatorname{deg}_{G}(v, W)$, where we may omit the index $G$ when the graph is clear from the context. The 1-density of $G$ is defined by $m_{1}(G)=\max \left\{\frac{e(F)}{v(F)-1}: F \subseteq G\right.$ with $\left.v(F) \geq 2\right\}$.

Moreover, given $p \in[0,1]$, an integer $k \geq 1$ and $k$ pairwise-disjoint sets of vertices $V_{1}, \ldots, V_{k}$, we denote by $G\left(V_{1}, \ldots, V_{k}, p\right)$ the random $k$-partite graph with parts $V_{1}, \ldots, V_{k}$, where each pair of vertices in two different parts forms an edge with probability $p$, independently of the other pairs.

Given a copy $F$ of the square of a path on $k$ vertices $P_{k}^{2}$, we let $v_{1}, v_{2}, \ldots, v_{k}$ be an ordering of the vertices of $F$ such that its edges are precisely $v_{i} v_{j}$, for each $i, j$ with $1 \leq|i-j| \leq 2$. We call $\left(v_{2}, v_{1}\right)$ and $\left(v_{k-1}, v_{k}\right)$ the end-tuples of $F$, we refer to $v_{i}$ as the $i$-th vertex of $F$, and we refer to $F$ as the square of the path $v_{1}, v_{2}, \ldots, v_{k}$. For simplicity we will talk about tuples $(u, v)$ from a set $V$, when implicitly meaning from $V^{2}$. 


\section{Tools}

We will repeatedly use the following concentration inequality due to Chernoff (see e.g. [19, Corollaries 2.3 and 2.4] and [18]).

Lemma 2.1 (Chernoff's inequality). Let $X$ be the sum of independent binomial random variables, then for any $\delta \in(0,1)$ we have

$$
\mathbb{P}[|X-\mathbb{E}[X]| \geq \delta \mathbb{E}[X]] \leq 2 \exp \left(-\frac{\delta^{2}}{3} \mathbb{E}[X]\right)
$$

and for any $k \geq 7 \cdot \mathbb{E}[X]$ we have $\mathbb{P}[X>k] \leq \exp (-k)$. More precisely, if $p$ is the success probability and there are $n$ summands we get

$$
\mathbb{P}[X \leq \mathbb{E}[X]-\delta n] \leq \exp (-D(p-\delta \| p) n),
$$

where $D(x \| y)=x \log \left(\frac{x}{y}\right)+(1-x) \log \left(\frac{1-x}{1-y}\right)$ is the relative entropy.

2.1. Subgraphs in random graphs. The following lemma is well-known and follows from a standard application of Janson's inequality (Lemma A.1).

Lemma 2.2. For any graph $F$ and any $\delta>0$, there exists $C>0$ such that the following holds for $p \geq C n^{-1 / m_{1}(F)}$. In the random graph $G(n, p)$ a.a.s. any set of $\delta$ n vertices contains a copy of $F$.

Note that $m_{1}\left(P_{k-1}\right)=1$ and $m_{1}\left(P_{k-1}^{2}\right)=\frac{2 k-3}{k-1}$ and, therefore, the bounds on $p$ given by Lemma 2.2 for the containment of a copy of $P_{k-1}$ and $P_{k-1}^{2}$ in any linear sized set are $p \geq C / n$ and $p \geq C n^{-(k-1) /(2 k-3)}$, respectively. In a breakthrough result Johansson, Kahn, and $\mathrm{Vu}$ [20] determined the threshold for covering all vertices of $G(n, p)$ with pairwise vertex-disjoint copies of $F$, for any strictly 1-balanced graph $F$, i.e. those graphs with 1-density strictly larger than that of any proper subgraph. We state their result below.

Theorem 2.3 (Johansson, Kahn, and $\mathrm{Vu}[20]$ ). Let $F$ be a graph such that $m_{1}\left(F^{\prime}\right)<$ $m_{1}(F)$ for all $F^{\prime} \subseteq F$ with $F^{\prime} \neq F$ and $v\left(F^{\prime}\right) \geq 2$. Then a.a.s. in $G(n, p)$ there are $\lfloor n / v(F)\rfloor$ pairwise vertex-disjoint copies of $F$, provided that $p \geq(\log n)^{1 / e(F)} n^{-1 / m_{1}(F)}$.

Often we will need to find combinations of squares of paths in $G(n, p)$ whose vertices must satisfy some additional constraints; for that, we will use the following lemma.

Lemma 2.4. For all integers $s \geq 1$ and $k \geq 2$, and any $0<\eta \leq 1$, there exists $C>0$ such that the following holds for $p \geq C n^{-(k-1) /(2 k-3)}$. Let $V$ be a vertex set of size $n$, $V_{1}, \ldots, V_{s}$ not necessarily disjoint subsets of $V$, and $H$ be a collection of tuples from $\prod_{i=1}^{s} V_{i}^{k}$. Then a.a.s. revealing $\Gamma=G(n, p)$ on $V$ gives the following. For any choice of $W_{i} \subseteq V_{i}$ with $i=1, \ldots, s$ such that $H^{\prime}=H \cap \prod_{i=1}^{s} W_{i}^{k}$ has size at least $\eta n^{s k}$, there is a tuple $\left(v_{i, j}: 1 \leq i \leq s, 1 \leq j \leq k\right)$ in $H^{\prime}$ with pairwise distinct vertices $v_{i, j} \in W_{i}$ for $i=1, \ldots, s$, $j=1, \ldots, k$, such that in $\Gamma$ for $i=1, \ldots, s$ we have the square of a path on $v_{i, 1}, \ldots, v_{i, k}$ and for $i=1, \ldots, s-1$ we have the edge $v_{i, k} v_{i+1,1}$.

Observe that the structure we get from Lemma 2.4 in $G(n, p)$ is given by $s$ copies of the square of a path on $k$ vertices and $s-1$ additional edges joining two consecutive such copies. Moreover when $k=2$ the structure is a path on $2 s$ vertices. In applications, we will often define several collections of tuples $H_{j} \subseteq \prod_{i=1}^{s} V_{j, i}^{k}$ for $j=1, \ldots, m$, and apply Lemma 2.4 to $H=\bigcup_{j=1}^{m} H_{j}$, where it is implicit that we apply it with $V_{i}=\bigcup_{j=1}^{m} V_{j, i}$. Also, we stress that, for a fixed $H$ and a typical revealed $G(n, p)$, the conclusion of the lemma holds for any large enough subset of the form $H \cap \prod_{i=1}^{s} W_{i}^{k}$ with $W_{i} \subseteq V_{i}$. In particular, we will be able to claim the existence of a tuple in each subcollection $H_{j}$, again provided 
they have the right size. The proof of this lemma is standard, it uses Janson's inequality, and is given in Appendix A.

We denote by $\vec{G}(n, p)$ the binomial random directed graph on vertex set $V$, where each tuple $(u, v) \in V^{2}$ with $u \neq v$ is a directed edge with probability $p$ independently of all the other choices. The next theorem will allow us to find a directed Hamilton cycle in $\vec{G}(n, p)$.

Theorem 2.5 (Angluin and Valiant [2]). There exists $C>0$ such that for $p \geq C \log n / n$ a.a.s. $\vec{G}(n, p)$ has a directed Hamilton cycle.

2.2. Regularity. We will use Szemerédi's Regularity Lemma [33] and some of its consequences. Before stating these, we introduce the relevant terminology. The density of a pair $(A, B)$ of disjoint sets of vertices is defined by

$$
d(A, B)=\frac{e(A, B)}{|A| \cdot|B|}
$$

and the pair $(A, B)$ is called $\varepsilon$-regular, if for all sets $X \subseteq A$ and $Y \subseteq B$ with $|X| \geq \varepsilon|A|$ and $|Y| \geq \varepsilon|B|$ we have $|d(A, B)-d(X, Y)| \leq \varepsilon$.

We will use the following well known result, that follows from definitions.

Lemma 2.6 (Minimum Degree Lemma). Let $(A, B)$ be an $\varepsilon$-regular pair with $d(A, B)=d$. Then, for every $Y \subseteq B$ with $|Y| \geq \varepsilon|B|$, the number of vertices from $A$ with degree into $Y$ less than $(d-\varepsilon)|Y|$ is at most $\varepsilon|A|$.

With $d \in[0,1]$, a pair $(A, B)$ is called $(\varepsilon, d)$-super-regular if, for all sets $X \subseteq A$ and $Y \subseteq B$ with $|X| \geq \varepsilon|A|$ and $|Y| \geq \varepsilon|B|$, we have $d(X, Y) \geq d$ and $\operatorname{deg}(a) \geq d|B|$ for all $a \in A$ and $\operatorname{deg}(b) \geq d|A|$ for all $b \in B$.

The following result is also well known and follows from the definition of superregularity and Lemma 2.6.

Lemma 2.7. Let $(A, B)$ be an $\varepsilon$-regular pair with $d(A, B)=d$. Then there exists $A^{\prime} \subseteq A$ and $B^{\prime} \subseteq B$ with $\left|A^{\prime}\right| \geq(1-\varepsilon)|A|$ and $\left|B^{\prime}\right| \geq(1-\varepsilon)|B|$ such that $\left(A^{\prime}, B^{\prime}\right)$ is a $(2 \varepsilon, d-3 \varepsilon)$ super-regular pair.

We will use the following well known degree form of the regularity lemma that can be derived from the original version [33].

Lemma 2.8 ([24]). For every $\varepsilon>0$ and integer $t_{0}$ there exists an integer $T>t_{0}$ such that for any graph $G$ on at least $T$ vertices and $d \in[0,1]$ there is a partition of $V(G)$ into $t_{0}<t+1 \leq T$ sets $V_{0}, \ldots, V_{t}$ and a subgraph $G^{\prime}$ of $G$ such that

(P1) $\left|V_{i}\right|=\left|V_{j}\right|$ for all $1 \leq i, j \leq t$ and $\left|V_{0}\right| \leq \varepsilon|V(G)|$,

(P2) $\operatorname{deg}_{G^{\prime}}(v) \geq \operatorname{deg}_{G}(v)-(d+\varepsilon)|V(G)|$ for all $v \in V(G)$,

(P3) the set $V_{i}$ is independent in $G^{\prime}$ for $1 \leq i \leq t$,

(P4) for $1 \leq i \leq j \leq t$ the pair $\left(V_{i}, V_{j}\right)$ is $\varepsilon$-regular in $G^{\prime}$ and has density either 0 or at least $d$.

The sets $V_{1}, \ldots, V_{t}$ are also called clusters and we refer to $V_{0}$ as the set of exceptional vertices. A partition $V_{0}, \ldots, V_{t}$ which satisfies $(\mathrm{P} 1)-(\mathrm{P} 4)$ is called an $(\varepsilon, d)$-regular partition of $G$. Given this partition, we define the $(\varepsilon, d)$-reduced graph $R$ for $G$, that is, the graph on vertex set $[t]$, in which $i j$ is an edge if and only if $\left(V_{i}, V_{j}\right)$ is an $\varepsilon$-regular pair in $G^{\prime}$ and has density at least $d$. 
2.3. Squares of paths in randomly perturbed graphs. We also need the following result that allows us to find multiple copies of the square of a short path in randomly perturbed graphs.

Lemma 2.9. For all integers $k \geq 2$ and $t \geq 1$, there exist $C, \gamma>0$ such that the following holds for any $1 \leq m \leq \gamma n$ and any $n$-vertex graph $G$ of minimum degree $\delta(G) \geq m$ and maximum degree $\Delta(G) \leq \gamma n$. For $p \geq C(\log n)^{1 /(2 k-3)} n^{-(k-1) /(2 k-3)}$, a.a.s. the perturbed graph $G \cup G(n, p)$ contains $t m+t$ pairwise vertex-disjoint copies of the square of a path on $k+1$ vertices.

The case $k=2$ and $t=1$ was already covered in [7, Theorem 2.4]. The general proof is similar and is given in Appendix A.

\section{PROOF OVERVIEW}

In this section we will sketch the proof of Theorem 1.1 and discuss a more general stability version of it. As already explained in the introduction, the cases $\alpha=0$ and $\alpha \geq \frac{1}{2}$ follow from known results. Moreover, the case $\alpha=\frac{1}{2}$ will follow from the monotonicity of the perturbed threshold, once we will have determined the perturbed threshold in the range $\alpha<\frac{1}{2}$. Therefore from now on, we can fix an integer $k \geq 2$ and assume $\alpha \in\left[\frac{1}{k+1}, \frac{1}{k}\right)$.

We start by discussing the idea of our embedding strategy and explaining how this leads to the threshold probabilities given in Theorem 1.1. We then turn to the arguments for the lower bound on $\hat{p}_{\alpha}$, and afterwards split the upper bound into two theorems (Theorems 3.5 and 3.4) depending on the structure of the dense graph $G_{\alpha}$ : an extremal case and a nonextremal one. Here, Theorem 3.4 provides a stability version of Theorem 1.1.

3.1. Strategy. Let $G$ be any $n$-vertex graph with minimum degree $\alpha n$ and $\alpha \in\left[\frac{1}{k+1}, \frac{1}{k}\right)$. Our goal is to find the square of a Hamilton cycle $C_{n}^{2}$ in the perturbed graph $G \cup G(n, p)$ and therefore we will use a decomposition of $E\left(C_{n}^{2}\right)$ into 'deterministic edges' (to be embedded to $G$ ) and 'random edges' (to be embedded to $G(n, p)$ ). To get the square of a path we would like vertex disjoint copies $F_{1}, \ldots, F_{t}$ of $P_{k}^{2}$ in the random graph $G(n, p)$ such that the following holds. For each $i=1, \ldots, t-1$, if we denote by $\left(x_{i}, y_{i}\right)$ and $\left(u_{i}, w_{i}\right)$ the end-tuples of $F_{i}$, then $w_{i} y_{i+1}$ is also an edge in $G(n, p)$. Moreover, there exist $t-1$ additional vertices $v_{1}, \ldots, v_{t-1}$ such that, for $i=1, \ldots, t-1$, all four edges $v_{i} u_{i}, v_{i} w_{i}, v_{i} x_{i+1}, v_{i} y_{i+1}$ are edges in $G$. This gives the square of a path on $t(k+1)-1$ vertices with edges from $G \cup G(n, p)$ (c.f. Figure 1).

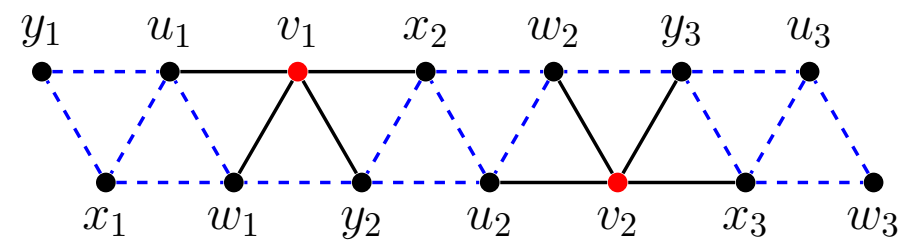

Figure 1 . The square of a path with end-tuples $\left(x_{1}, y_{1}\right)$ and $\left(u_{3}, w_{3}\right)$ with our decomposition into random (dashed blue) and deterministic (black) edges for $k=4$.

Note that by requiring the edge $w_{t} x_{1}$ from $G(n, p)$ and adding another vertex $v_{t}$ joined to $u_{t}, w_{t}, x_{1}, y_{1}$ in $G$, we get the square of a cycle on $t(k+1)$ vertices. However for parity reasons this may not cover all $n$ vertices. Hence in order to find the square of a Hamilton cycle and for some additional technical reasons, our proof(s) will allow some of $F_{1}, \ldots, F_{t}$ to be the squares of paths of different lengths. 
This decomposition of $C_{n}^{2}$ already justifies the probabilities that appear in Theorem 1.1: indeed, $n^{-(k-1) /(2 k-3)}$ is the threshold in $G(n, p)$ for a linear number of copies of $P_{k}^{2}$ (by a standard application of Janson's inequality), while $n^{-(k-1) /(2 k-3)}(\log n)^{1 /(2 k-3)}$ is the threshold in $G(n, p)$ for the existence of a $P_{k}^{2}$-factor (this follows from Theorem 2.3).

3.2. Lower bounds. For any $\alpha \in(0,1 / 2)$, let $H_{\alpha}$ be the complete bipartite $n$-vertex graph with vertex classes $A$ and $B$ of size $\alpha n$ and $(1-\alpha) n$, respectively. The following two propositions provide lower bounds on $\hat{p}_{\alpha}$ for $\alpha \in\left(\frac{1}{k+1}, \frac{1}{k}\right)$ and $\alpha=\frac{1}{k+1}$. Their proofs are standard and we only give a sketch.

Proposition 3.1. Let $\alpha \in\left(\frac{1}{k+1}, \frac{1}{k}\right)$. Then there exists $0<c<1$ such that $H_{\alpha} \cup G(n, p)$ a.a.s. does not contain a copy of $C_{n}^{2}$, provided $p \leq c n^{-(k-1) /(2 k-3)}$.

Sketch of the proof of Proposition 3.1. In $B$ there are a.a.s. at most $2 c n$ copies of $P_{k}^{2}$ (by an upper tail bound on the distribution of small subgraphs [34]) and at most $o(n)$ copies of $P_{k+1}^{2}$ (by the first moment method). Assume for contradiction that there is an embedding of $C_{n}^{2}$ into $H_{\alpha} \cup G(n, p)$. Then an $\alpha$-fraction of the vertices is mapped into $A$ and, because of the bound on the number of $P_{k+1}^{2}$ in $B$, two such vertices can only rarely be of distance more than $k+1$ in $C_{n}^{2}$. From this it follows that there are at least $\frac{1-\alpha k}{2} n$ copies of $P_{k}^{2}$ in $B$, which is a contradiction if $c<\frac{1-\alpha k}{4}$.

Proposition 3.2. Let $\alpha=\frac{1}{k+1}$. Then $H_{\alpha} \cup G(n, p)$ a.a.s. does not contain a copy of $C_{n}^{2}$, provided $p \leq \frac{1}{2} n^{-(k-1) /(2 k-3)}(\log n)^{1 /(2 k-3)}$.

Sketch of the proof of Proposition 3.2. Let $c=\frac{1}{2 k}$. Then a.a.s. (by the first moment method) at least $n^{1-2 c}$ vertices from $B$ are not contained in any copy of $P_{k}^{2}$ within $B$, and $B$ contains at most $n^{1-c}$ copies of $P_{k+1}^{2}$. Therefore, in any embedding of $C_{n}^{2}$, the distance between two vertices mapped into $A$ can only $n^{1-c}$ often be larger than $k+1$, but exactly one in $k+1$ vertices is mapped into $A$. This implies that all but $n^{1-c}$ vertices from $B$ are contained in a copy of $P_{k}^{2}$ within $B$, which gives a contradiction.

3.3. Proof of Theorem 1.1 and stability. As mentioned before, for the proof of Theorem 1.1 we distinguish between an extremal case, when the deterministic graph $G$ is close to $H_{1 /(k+1)}$, i.e. the complete bipartite $n$-vertex graph with vertex classes of size $\frac{1}{k+1} n$ and $\frac{k}{k+1} n$, and a non-extremal case. It turns out that the additional $(\log n)^{1 /(2 k-3)}$ term in the perturbed threshold at $\alpha=\frac{1}{k+1}$ is only necessary in the extremal case. The next definition formalises what we mean by close.

Definition $3.3((\alpha, \beta)$-stable). For $\alpha, \beta>0$ we say that an $n$-vertex graph $G$ is $(\alpha, \beta)$ stable if there exists a partition of $V(G)$ into two sets $A$ and $B$ of sizes $|A|=(\alpha \pm \beta) n$ and $|B|=(1-\alpha \pm \beta) n$ such that the minimum degree of the bipartite subgraph $G[A, B]$ of $G$ induced by $A$ and $B$ is at least $\frac{1}{4} \alpha n$, all but at most $\beta n$ vertices from $A$ have degree at least $|B|-\beta n$ into $B$, all but at most $\beta n$ vertices from $B$ have degree at least $|A|-\beta n$ into $A$, and $G[B]$ contains at most $\beta n^{2}$ edges.

The following stability theorem treats the non-extremal case of Theorem 1.1.

Theorem 3.4 (Stability). For every $k \geq 2$ and every $0<\beta<\frac{1}{6 k}$, there exists $\gamma>0$ and $C>0$ such that the following holds. Let $G$ be any n-vertex graph with minimum degree at least $\left(\frac{1}{k+1}-\gamma\right) n$ that is not $\left(\frac{1}{k+1}, \beta\right)$-stable. Then $G \cup G(n, p)$ a.a.s. contains the square of a Hamilton cycle, provided that $p \geq \mathrm{Cn}^{-(k-1) /(2 k-3)}$.

Only when the graph $G$ is stable we need the $(\log n)^{1 /(2 k-3)}$-term. This case is treated by the following theorem. 
Theorem 3.5 (Extremal case). For every $k \geq 2$ there exists $\beta>0$ and $C>0$ such that the following holds. Let $G$ be any $n$-vertex graph with minimum degree at least $\frac{1}{k+1} n$ that is $\left(\frac{1}{k+1}, \beta\right)$-stable. Then $G \cup G(n, p)$ a.a.s. contains the square of a Hamilton cycle, provided that $p \geq C n^{-(k-1) /(2 k-3)}(\log n)^{1 /(2 k-3)}$.

We sketch the ideas for the proof of these two theorems in the following two subsections. Together with the lower bounds provided in Propositions 3.1 and 3.2, Theorem 3.4 and 3.5 imply Theorem 1.1 for $\alpha \in\left[\frac{1}{k+1}, \frac{1}{k}\right)$ with $k \geq 2$.

3.4. Overview of the proof of Theorem 3.5. For the extremal case, suppose that $G$ is an $n$-vertex $\left(\frac{1}{k+1}, \beta\right)$-stable graph, and let $p \geq C(\log n)^{1 /(2 k-3)} n^{-(k-1) /(2 k-3)}$. The definition of stability (Definition 3.3) gives a partition $A \cup B$ of $V(G)$ in which the size of $B$ is roughly $k$ times the size of $A$, the minimum degree of $G[A, B]$ is at least $\alpha n / 4$, and all but few vertices of $A$ ( $B$, respectively) are adjacent to all but few vertices of $B(A$, respectively). Our proof will follow three steps.

In the first step, we would like to embed copies $F_{i}$ of $P_{k}^{2}$ into $B$ and vertices $v_{i}$ into $A$, following the decomposition described above. However, this is only possible if $|B|=k|A|$ and, therefore, we first embed squares of short paths of different lengths to ensure this parity condition in the remainder. We find a family $\mathcal{F}_{1}$ of copies of squares of paths with end-tuples in $B$, such that after removing the vertices $V_{1}=\bigcup_{F \in \mathcal{F}} V(F)$, we are left with two sets $A_{1}=A \backslash V_{1}$ and $B_{1}=B \backslash V_{1}$ with $\left|B_{1}\right|=k\left(\left|A_{1}\right|-\left|\mathcal{F}_{1}\right|\right)$. Note that we require $\left|B_{1}\right|=k\left(\left|A_{1}\right|-\left|\mathcal{F}_{1}\right|\right)$ insted of $\left|B_{1}\right|=k\left|A_{1}\right|$, because each square path in $\mathcal{F}_{1}$ still needs to be connected into the final square of a Hamilton cycle, and for each of these connections we shall use one vertex in $A_{1}$. The precise way we find $\mathcal{F}_{1}$ depends on the sizes of $A$ and $B$, but in all cases we will ensure that the vertices in the end-tuples of each $F \in \mathcal{F}_{1}$ are neighbours of all but few vertices of $A$. When $|B|>\frac{k}{k+1} n$, the family $\mathcal{F}_{1}$ consists of copies of $P_{k+1}^{2}$ inside of $B$. Its existence is guaranteed by Lemma 2.9 using the minimum degree of $G[B]$. When $|B| \leq \frac{k}{k+1} n$, the family $\mathcal{F}_{1}$ consists of copies of $P_{2 k+3}^{2}$ with all vertices in $B$, except the $(k+1)$-st and $(k+3)$-rd, that belong to $A$.

Our second step is to cover the vertices in $A_{1}$ and $B_{1}$ that do not have a high degree to the other part. For this we will find another family $\mathcal{F}_{2}$ of copies of squares of paths with end-tuples in $B$. For any vertex $v$ in $A_{1}$ with small degree into $B_{1}$, we find a copy of $P_{2 k+1}^{2}$ with $v$ being the $(k+1)$-st vertex and all the remaining vertices belonging to $B_{1}$. Similarly, for any vertex $v$ in $B_{1}$ with small degree into $A_{1}$, we find a copy of $P_{3 k+2}^{2}$ consisting of three copies of $P_{k}^{2}$ in $B$ connected by edges and two vertices from $A_{1}$, where $v$ is in the middle copy of $P_{k}^{2}$. We need that $v$ is in the middle copy, because then we can again ensure that the end-tuples of each $F \in \mathcal{F}_{2}$ see all but few vertices of $A$. Moreover, with $V_{2}=\bigcup_{F \in \mathcal{F}_{2}} V(F)$ and $A_{2}=A_{1} \backslash V_{2}$ and $B_{2}=B_{1} \backslash V_{2}$, we have $\left|B_{2}\right|=k\left(\left|A_{2}\right|-\left|\mathcal{F}_{1}\right|-\left|\mathcal{F}_{2}\right|\right)$.

At this point, each of the vertices in $A_{2}$ ( $B_{2}$, respectively) is adjacent to all but few vertices of $B_{2}\left(A_{2}\right.$, respectively) and we kept the parity intact. In the third step, we let $\mathcal{F}_{3}$ be pairwise disjoint copies of $P_{k}^{2}$ covering $B_{2}$, which is possible by Theorem 2.3 with our $p$ and because $\left|B_{2}\right|$ is divisible by $k$.

We let $\mathcal{F}=\mathcal{F}_{1} \cup \mathcal{F}_{2} \cup \mathcal{F}_{3}$ and, for each $F \in \mathcal{F}$, denote its end-tuples by $\left(y_{F}, x_{F}\right)$ and $\left(u_{F}, w_{F}\right)$. We now reveal additional edges of $G(n, p)$ and encode their presence in an auxiliary directed graph $\mathcal{T}$ on vertex set $\mathcal{F}$ as follows. There is a directed edge $\left(F, F^{\prime}\right)$ if and only if the edge $w_{F} x_{F^{\prime}}$ appears in $G(n, p)$. It is easy to see that all directed edges in $\mathcal{T}$ are revealed with probability $p$ independently of all the others and, therefore, we can find a directed Hamilton $\vec{C}$ cycle in $\mathcal{T}$ with Theorem 2.5. We finally match to each edge $\left(F, F^{\prime}\right)$ of $\vec{C}$ a vertex $v \in A_{2}$ such that $u_{F}, w_{F}, x_{F^{\prime}}, y_{F^{\prime}}$ are all neighbours of $v$ in the graph $G$. Owing to the high minimum degree conditions, that this is possible easily 
follows from Hall's matching theorem. Thus we get the square of a Hamilton cycle, as wanted.

3.5. Overview of of the proof of Theorem 3.4. Assume that $G$ is not $\left(\frac{1}{k+1}, \beta\right)$-stable and let $p \geq C n^{-(k-1) /(2 k-3)}$. Then we apply the regularity lemma to $G$ and we use the following lemma on its reduced graph $R$.

Lemma 3.6 (Lemma 4.4 in [7]). For any integer $k \geq 2$, and $0<\beta<\frac{1}{12}$ there exists $d>0$ such that the following holds for any $0<\varepsilon<d / 4,4 \beta \leq \alpha \leq \frac{1}{3}$, and $t \geq \frac{10}{d}$. Let $G$ be an $n$ vertex graph with minimum degree $\delta(G) \geq\left(\alpha-\frac{1}{2} d\right) n$ that is not $(\alpha, \beta)$-stable and let $R$ be the $(\varepsilon, d)$-reduced graph for some $(\varepsilon, d)$-regular partition $V_{0}, \ldots, V_{t}$ of $G$. Then $R$ contains a matching $M$ of size $(\alpha+2 k d) t$.

In fact, [7, Lemma 4.4] is weaker as, under the same assumptions, it gives a matching of size $(\alpha+2 d) t$, but Lemma 3.6 follows from a straightforward adaption of its proof, which in turn builds on ideas from [4]. With Lemma 3.6, it is not hard to show that the reduced graph $R$ can be vertex-partitioned into copies of stars $K_{1, k}$ and matching edges $K_{1,1}$, such that there are not too many stars. We would like to cover the clusters corresponding to each such star and matching edge with the square of a Hamilton path, and then connect these square paths to get the square of a Hamilton cycle. However, since we want to avoid the additional log-term in the probability we are working with, for this strategy to work in the randomly perturbed graph, we need that in each star the centre cluster is larger than the other clusters. Moreover, to ensure that we can connect the Hamilton paths, we need to setup some connections between the stars and matching edges.

Therefore, we first remove some vertices from the leaf clusters of each star to make it unbalanced and ensure that all pairs are super-regular. Then we label the stars and matching edges arbitrarily as $Q_{1}, \ldots, Q_{s}$, and for $i=1, \ldots, s$ find a copy $F_{i}$ of $P_{6}^{2}$ with end-tuples in leaf clusters of $Q_{i}$ and $Q_{i+1}$ (where indices are modulo $s$ ). More precisely, for each star $Q_{i}$, one end-tuple of $F_{i-1}$ and one of $F_{i}$ belong to the centre cluster of $Q_{i}$, while for each matching edge $Q_{i}$, each of its two cluster contains exactly one end-tuple, one from $F_{i-1}$ and the other from $F_{i}$. We will refer to these squares paths as the connecting (square) paths. Let $V_{0}$ be the sets of vertices no longer contained in any of the stars or matching edges. We cover each $v \in V_{0}$ by appending $v$ to one of the connecting paths. Here we use that any vertex $v \in V_{0}$ has degree at least $\left(\frac{1}{k+1}-\alpha\right) n$ and, as we do not have too many stars, the vertex $v$ has also many neighbours in clusters which are not centres of stars. This is crucial, because it allows us to ensure that the relations between the sizes of the sets in any star are suitable for an application of the following lemma, which is the main technical ingredient in the proof.

Lemma 3.7. For any $k \geq 2$ and any $0<\delta^{\prime} \leq d<1$ there exist $\delta_{0}, \delta_{1}, \varepsilon>0$ with $\delta^{\prime} \geq \delta_{0}>2 \delta_{1}$ and $C>0$ such that the following holds. Let $V, U_{1}, \ldots, U_{k}$ be pairwise disjoint sets such that $|V|=n+4,\left(1-\delta_{0}\right) n \leq\left|U_{1}\right|=\cdots=\left|U_{k}\right| \leq\left(1-\delta_{1}\right) n$ and $n-\left|U_{1}\right| \equiv 0(\bmod 6 k-2)$. Suppose that $\left(V, U_{i}\right)$ are $(\varepsilon, d)$-super-regular pairs with respect to a graph $G$ for each $i=1, \ldots, k$, and $\left(x, x^{\prime}\right)$ and $\left(y, y^{\prime}\right)$ are two tuples from $V$ such that both tuples have $\frac{1}{2} d^{2} n$ common neighbours in $U_{i}$ for each $i=1, \ldots, k$. Furthermore, let $G(V, p)$ and $G\left(U_{1}, \ldots, U_{k}, p\right)$ be random graphs with $p \geq C n^{-(k-1) /(2 k-3)}$.

Then a.a.s. there exists the square of a Hamilton path in $G\left[V, U_{1}, \ldots, U_{k}\right] \cup G(V, p) \cup$ $G\left(U_{1}, \ldots, U_{k}, p\right)$ covering $V, U_{1}, \ldots, U_{k}$, and with end-tuples $\left(x, x^{\prime}\right)$ and $\left(y, y^{\prime}\right)$, in which the edges $x x^{\prime}, y y^{\prime}$ are not required. 
This implies that for any star $Q_{i}$ we can connect the end-tuples of $F_{i-1}$ and $F_{i}$ which belong to the centre cluster of $Q_{i}$, while covering all vertices in the clusters of $Q_{i}$. We emphasise again that, to avoid log-terms, it is crucial that the centre cluster is larger than the leaf clusters. Similarly for the matching edges we use the following lemma.

Lemma 3.8. For any $0<d<1$ there exist $\varepsilon>0$ and $C>0$ such that the following holds for sets $U, V$ with $|V|=n$ and $\frac{3}{4} n \leq|U| \leq n$. Let $(U, V)$ be an $(\varepsilon, d)$-super-regular with respect to a graph $G$ and $\left(x, x^{\prime}\right)$ and $\left(y, y^{\prime}\right)$ be tuples from $V$ and $U$, respectively, such that the vertices from the tuples have $\frac{1}{2} d^{2} n$ common neighbours in $U$ and $V$, respectively. If $G(U, p), G(V, p)$ are random graphs with $p \geq C n^{-1}$, then a.a.s. there exists the square of a Hamilton path in $G[U, V] \cup G(U, p) \cup G(V, p)$ covering $U, V$ with end-tuples $\left(x, x^{\prime}\right)$ and $\left(y, y^{\prime}\right)$, in which the edges $x x^{\prime}$ and $y y^{\prime}$ are not required.

Together this gives the square of a Hamilton cycle in $G \cup G(n, p)$. We will give the proof of Lemma 3.7 and 3.8 in Section 7.

\section{Proof of Theorem 3.5}

Proof of Theorem 3.5. Given an integer $k \geq 2$, we let $C_{2}$ and $0<\gamma \leq 1$ be given by Lemma 2.9 for input $k$ and $t=k+1$. We let $C_{4}$ be given by Theorem 2.5 and set $C=4 C_{2}+8 k C_{4}+8$. Next, we let $0<\beta \leq \frac{1}{100 k^{3}} \gamma$. Given $n$, let $0 \leq a \leq k$ be such that $n=(k+1)\left\lfloor\frac{1}{k+1} n\right\rfloor+a$ and $p \geq C(\log n)^{1 /(2 k-3)} n^{-(k-1)(2 k-3)}$. We reveal a subgraph of $G(n, p)$ in four rounds $G_{i} \sim G\left(n, \frac{1}{4} p\right)$ for $i=1,2,3,4$. By Lemma 2.2 and a union bound, we can a.a.s. assume that

$G_{1}$ contains a copy of the graph $F$ in any vertex-set of size at least $\beta n$,

where $F$ is any graph on at most $4 k$ vertices with $m_{1}(F) \leq m_{1}\left(P_{k}^{2}\right)=\frac{2 k-3}{k-1}$.

Let $G$ be an $n$-vertex graph with minimum degree at least $\frac{1}{k+1} n$ that is $\left(\frac{1}{k+1}, \beta\right)$-stable. Then there exists a partition of $V(G)$ into $A$ and $B$ that satisfies Definition 3.3. As outlined in Section 3.4 our proof will consist of three steps. We will successively build parts of the square of a Hamilton cycle, first covering some vertices to balance the partition, then covering vertices of low degree to the other side and, then, covering the remaining vertices. Finally we will connect these parts into the square of a Hamilton cycle.

Balancing the partition. Our goal is to find a family $\mathcal{F}_{1}$ of pairwise disjoint copies of squares of paths with end-tuples in $B$, such that the size of the set $V_{1}=\bigcup_{F \in \mathcal{F}_{1}} V(F)$ is smaller than $3 k^{2} \beta n$, and after removing the vertices of $V_{1}$ we are left with two sets

$$
A_{1}=A \backslash V_{1} \text { and } B_{1}=B \backslash V_{1} \text { such that }\left|B_{1}\right|=k\left(\left|A_{1}\right|-\left|\mathcal{F}_{1}\right|\right) \text {. }
$$

We distinguish between the cases $|A|=\left\lfloor\frac{n}{k+1}\right\rfloor+m$ with $1 \leq m \leq \beta n$ and $|A|=\left\lfloor\frac{n}{k+1}\right\rfloor-m$ with $0 \leq m \leq \beta n$. Suppose first that $|A|=\left\lfloor\frac{n}{k+1}\right\rfloor+m$ for some $1 \leq m \leq \beta n$. In this case we want $\left|\mathcal{F}_{1}\right|=m$ and the family $\mathcal{F}_{1}$ will consist of $m-1$ copies of $P_{3 k+2}^{2}$ and one copy of $P_{3 k+2+a}^{2}$, such that for each of these $m$ copies, exactly three vertices are in $A$, both end-tuples are in $B$ and each end-tuple has at least $|A|-2 \beta n$ common neighbours in $A$.

We can do this greedily in $G \cup G_{1}$. Assume that during this process we have to find a copy of $P_{3 k+2}^{2}$ or $P_{3 k+2+a}^{2}$, i.e. a copy of $P_{3 k+b}^{2}$ for some $2 \leq b \leq k+2$, such that the above conditions are satisfied. There are three vertices $v_{1}, v_{2}$ and $v_{3}$ in $P_{3 k+b}^{2}$, such that none of them is in an end-tuple of $P_{3 k+b}$, they do not induce a triangle in $P_{3 k+b}^{2}$, and the subgraph $H=P_{3 k+b}^{2} \backslash\left\{v_{1}, v_{2}, v_{3}\right\}$ satisfies $m_{1}(H) \leq m_{1}\left(P_{k}^{2}\right)$ (see Figure 2). We can avoid an induced triangle, because there are at least $3 k+b-4 \geq 4$ vertices to choose from that are not in end-tuples, and guarantee the bound on the density because, when distributing the three vertices evenly, the longest square of a path in $H$ has at most $\lceil(3 k+b-3) / 4\rceil \leq k$ vertices. 
We remark that we can always ask $\left\{v_{1}, v_{2}, v_{3}\right\}$ to be an independent set in $P_{3 k+b}^{2}$ when $k>2$.

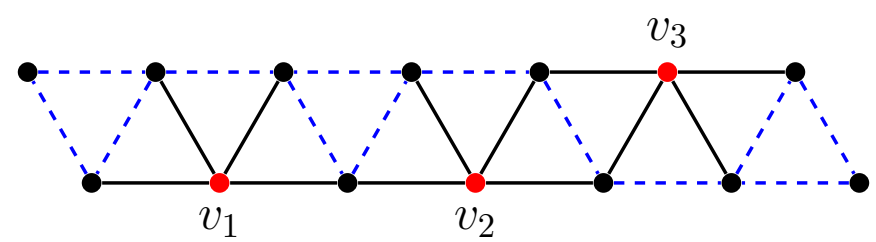

FiguRE 2. Subgraph $H$ (dashed blue) obtained from $P_{3 k+b}$ after removing three vertices $v_{1}, v_{2}, v_{3}$ (red) for $b=k+2$ and $k=3$. The 1-density of $H$ is the same as $P_{k}^{2}$.

Then we find a copy of $P_{3 k+b}^{2}$, by embedding the vertices $v_{1}, v_{2}, v_{3}$ in $A$ and the other vertices in $B$ in the following way. Let $A^{\prime} \subseteq A$ be the set of vertices of $A$ that have degree at least $|B|-\beta n$ into $B$ and have not yet been covered, and observe that $\left|A^{\prime}\right| \geq$ $|A|-\beta n-3\left|\mathcal{F}_{1}\right| \geq \beta n$. Then, since $m_{1}\left(P_{3}\right)=1 \leq m_{1}\left(P_{k}^{2}\right)$ and given (1), the random graph $G_{1}\left[A^{\prime}\right]$ contains a path on three vertices $u_{1}, u_{2}, u_{3}$. Next, let $B^{\prime} \subseteq B$ be the set of vertices of $B$ that have degree at least $|A|-\beta n$ into $A$, are common neighbours of $u_{1}, u_{2}, u_{3}$ and have not yet been covered, and observe that $\left|B^{\prime}\right| \geq|B|-\beta n-3 \beta n-(3 k+k+2-3)\left|\mathcal{F}_{1}\right| \geq \beta n$. Since $m_{1}(H)=m_{1}\left(P_{k}^{2}\right)$, using again (1), the random graph $G_{1}\left[B^{\prime}\right]$ contains a copy of $H$, that together with the vertices $u_{1}, u_{2}, u_{3}$ and some edges from $G$ gives a copy of $P_{3 k+b}^{2}$. In particular, since each vertex in the end-tuples of $P_{3 k+b}$ is embedded in $B^{\prime}$, then, by definition of $B^{\prime}$, each end-tuple has at least $|A|-2 \beta n$ common neighbours in $A$. In view of (2), we get that $\left|A_{1}\right|=\left\lfloor\frac{n}{k+1}\right\rfloor-2 m$,

$$
\left|B_{1}\right|=n-|A|-(3 k-1) m-a=k\left\lfloor\frac{n}{k+1}\right\rfloor-3 k m=k\left(\left|A_{1}\right|-\left|\mathcal{F}_{1}\right|\right),
$$

and $\left|V_{1}\right|=(m-1)(3 k+2)+3 k+2+a \leq(4 k+3) m \leq 3 k^{2} \beta n$.

Now suppose that $|A|=\left\lfloor\frac{n}{k+1}\right\rfloor-m$ for some $0 \leq m \leq \beta n$. In this case, the family $\mathcal{F}_{1}$ will consist of some copies of $P_{k+1}^{2}$ and some copies of $P_{2 k+1}^{2}$, such that, for each copy, all vertices are in $B$ and each end-tuple has at least $|A|-8 k^{2} \beta n$ common neighbours in $A$; in particular, we do no touch the set $A$. We start from $\mathcal{F}_{1}=\varnothing$ and let $B^{\star}=\{v \in$ $B: d(v, B) \geq 4 k^{2} \beta n$ \} be the set of vertices in $B$ with high degree to $B$ in $G$. Since by Definition 3.3 we have $e(G[B]) \leq \beta n^{2}$, then $\left|B^{\star}\right| \leq \frac{n}{2 k^{2}}$. Moreover if $v \in B \backslash B^{\star}$, then $d(v, A) \geq \delta(G)-d(v, B) \geq \frac{n}{k+1}-4 k^{2} \beta n \geq|A|-4 k^{2} \beta n$. With $m_{0}=\max \left\{m-\left|B^{\star}\right|, 0\right\}$, we have $\delta\left(G\left[B \backslash B^{\star}\right]\right) \geq m_{0}$, as

$$
\delta\left(G\left[B \backslash B^{\star}\right]\right) \geq \frac{n}{k+1}-|A|-\left|B^{\star}\right|=\frac{n}{k+1}-\left\lfloor\frac{n}{k+1}\right\rfloor+m-\left|B^{\star}\right| \geq m-\left|B^{\star}\right| .
$$

Moreover, by definition of $B^{\star}$, we have $\Delta\left(G\left[B \backslash B^{\star}\right]\right) \leq 4 k^{2} \beta n \leq \gamma\left|B \backslash B^{\star}\right|$, where the last inequality follows from the choice of $\beta$ and $\left|B \backslash B^{\star}\right| \geq\left(\frac{k}{k+1}-\frac{1}{2 k^{2}}\right) n$. Therefore we can use Lemma 2.9 with parameters $k$ and $t=k+1$ and we a.a.s. find $(k+1) m_{0}+a$ pairwise disjoint copies of $P_{k+1}^{2}$ in $\left(G \cup G_{2}\right)\left[B \backslash B^{\star}\right]$, which we add to $\mathcal{F}_{1}$. All the vertices of such copies belong to $B \backslash B^{\star}$ and thus in particular each end-tuple has at least $|A|-8 k^{2} \beta n$ common neighbours in $A$.

Next we want to find $m-m_{0} \leq \min \left\{\left|B^{\star}\right|, m\right\}$ copies of $P_{2 k+1}^{2}$ in $B$ disjoint from any graph already in $\mathcal{F}_{1}$. First observe that the graph $H$ obtained by taking the disjoint union of two copies of $P_{k}^{2}$ with the addition of the edge between the last vertex of the first copy and the first vertex of the second copy satisfies $m_{1}(H) \leq m_{1}\left(P_{k}^{2}\right)$. The graph $H$ will be embedded in $G_{1}$ and we will turn that into an embedding of $P_{2 k+1}^{2}$, by adding a vertex and four edges of $G$. We can again do this greedily in $G \cup G_{1}$. First we remove vertices 
from $B^{\star}$ such that $\left|B^{\star}\right|=m-m_{0} \leq m \leq \beta n$. Then we let $B^{\prime}$ be the set of vertices in $B$ with less than $|A|-\beta n$ neighbours in $A$, and note that $\left|B^{\prime}\right| \leq \beta n$. We then pick a vertex $w$ from $B^{\star}$, not yet covered, and denote by $N_{w}$ the set of neighbours of $w$ in $B \backslash\left(B^{\star} \cup B^{\prime}\right)$ in the graph $G$, that have not yet been covered. Then

$$
\left|N_{w}\right| \geq 4 k^{2} \beta n-\left|B^{\prime}\right|-\left|B^{\star}\right|-\left((k+1) m_{0}+a\right)(k+1)-\left(m-m_{0}\right)(2 k+1-1) \geq \beta n .
$$

Therefore, since $m_{1}(H) \leq m_{1}\left(P_{k}^{2}\right)$ and using (1), the random graph $G_{1}\left[N_{w}\right]$ contains a copy of this $H$, that together with $w$ and four edges from $G$, gives a copy of $P_{2 k+1}^{2}$ as desired. The end-tuples of this copy belong to $B \backslash B^{\prime}$ and thus each of them has at least $|A|-2 \beta n$ common neighbours in $A$. Once this is done, in view of (2), we indeed get $\left|A_{1}\right|=|A|=\left\lfloor\frac{1}{k+1} n\right\rfloor-m$,

$$
\begin{array}{r}
\left|B_{1}\right|=n-|A|-(k+1)\left((k+1) m_{0}+a\right)-(2 k+1)\left(m-m_{0}\right) \\
=k\left(\left\lfloor\frac{n}{k+1}\right\rfloor-a-2 m-k m_{0}\right)=k\left(\left|A_{1}\right|-\left|\mathcal{F}_{1}\right|\right),
\end{array}
$$

and $\left|V_{1}\right|=\left((k+1) m_{0}+a\right)(k+1)+\left(m-m_{0}\right)(2 k+1) \leq(k+1)^{2} m+k(k+1) \leq 3 k^{2} \beta n$. This finishes the first step of our proof.

Covering low degree vertices. In this step the goal is to find a family $\mathcal{F}_{2}$ of pairwise disjoint copies of $P_{2 k+1}^{2}$ and $P_{3 k+2}^{2}$ that cover all vertices from $A$ (respectively $B$ ) that do not have high degree to $B$ (respectively $A$ ) and such that, for each copy, each end-tuple is in $B$ and has at least $|A|-16 k^{2} \beta n$ common neighbours in $A$. We will do this such that after removing $V_{2}=\bigcup_{F \in \mathcal{F}_{2}} V(F)$ we are left with two sets

$$
A_{2}=A_{1} \backslash V_{2} \text { and } B_{2}=B_{1} \backslash V_{2} \text { such that }\left|B_{2}\right|=k\left(\left|A_{2}\right|-\left|\mathcal{F}_{1}\right|-\left|\mathcal{F}_{2}\right|\right) \text {. }
$$

We let $A^{\prime}=\left\{v \in A_{1}: d(v, B) \leq|B|-\beta n\right\}$ and $B^{\prime}=\left\{v \in B_{1}: d(v, A) \leq|A|-8 k^{2} \beta n\right\}$, and note that $\left|A^{\prime}\right| \leq \beta n$ and $\left|B^{\prime}\right| \leq \beta n$. Let $\mathcal{F}_{2}=\varnothing$. We start by taking care of the vertices in $A^{\prime}$ and we cover each of them with a copy of $P_{2 k+1}^{2}$ with all other vertices in $B_{1} \backslash B^{\prime}$. For $u \in A^{\prime}$, let $N_{u}$ be the set of neighbours of $u$ in $B_{1} \backslash B^{\prime}$ in $G$ that are not yet covered by any graph in $\mathcal{F}_{2}$ and observe that

$$
\left|N_{u}\right| \geq d(u, B)-\left|B \backslash B_{1}\right|-\left|B^{\prime}\right|-2 k\left|\mathcal{F}_{2}\right| \geq \frac{n}{4(k+1)}-3 k^{2} \beta n-\beta n-2 k \beta n \geq \beta n,
$$

where we used that $d(u, B) \geq \frac{n}{4(k+1)}$ as $G$ is $\left(\frac{1}{k+1}, \beta\right)$-stable (see Definition 3.3). Note that the graph $H$ obtained by taking the union of two copies of $P_{k}^{2}$ with the addition of an edge between two end-vertices satisfies $m_{1}(H) \leq m_{1}\left(P_{k}^{2}\right)$. Therefore, using (1), the random graph $G_{1}\left[N_{u}\right]$ contains a copy of $H$ and together with $u$ and four edges of $G$, this gives the desired copy of $P_{2 k+1}^{2}$. Both end-tuples of this copy of $P_{2 k+1}^{2}$ belong to $B_{1} \backslash B^{\prime}$ and, thus, have at least $|A|-16 k^{2} \beta n$ common neighbours in $A$. We add this copy of $P_{2 k+1}^{2}$ to $\mathcal{F}_{2}$ and we continue until we cover all vertices of $A^{\prime}$.

Now we cover each vertex from $B^{\prime}$ with a copy of $P_{3 k+2}^{2}$, where each copy uses one vertex from $B^{\prime}$, two vertices from $A_{1} \backslash A^{\prime}$ and the other $3 k-1$ vertices from $B_{1} \backslash B^{\prime}$. Let $w \in B^{\prime}$ and $u_{1}, u_{2} \in A_{1} \backslash A^{\prime}$ be vertices not yet covered. We denote by $N_{w}$ the subset of $B_{1} \backslash B^{\prime}$ which contains the common neighbours of $w, u_{1}, u_{2}$ in $G$ that are not yet covered. Observe that the definitions of $A^{\prime}$ and $B^{\prime}$ give

$$
\begin{aligned}
\left|N_{w}\right| & \geq(\delta(G)-d(w, A))-\left(|B|-d\left(u_{1}, B\right)\right)-\left(|B|-d\left(u_{2}, B\right)\right)-\left|B \backslash B_{1}\right|-3 k\left|B^{\prime}\right|-2 k\left|A^{\prime}\right| \\
& \geq \frac{n}{k+1}-\left(|A|-8 k^{2} \beta n\right)-\beta n-\beta n-3 k^{2} \beta n-3 k \beta n-2 k \beta n \geq \beta n .
\end{aligned}
$$

Similarly as above, using (1), the random graph $G_{1}\left[N_{w}\right]$ contains a copy of a graph $H$ on $3 k-1$ vertices, that together with $w, u_{1}, u_{2}$ and some edges from $G$, gives a copy of $P_{3 k+2}^{2}$. The end-tuples of the copy of $P_{3 k+2}^{2}$ belong to $B_{1} \backslash B^{\prime}$ and, thus, have have at least 
$|A|-16 k^{2} \beta n$ common neighbours in $A$. We add the copy of $P_{3 k+2}^{2}$ to $\mathcal{F}_{2}$ and repeat until all of $B^{\prime}$ is covered. We then get (3), because of (2) and since for each graph added to $\mathcal{F}_{2}$ the ratio of vertices removed from $A_{1}$ and $B_{1}$ is one to $2 k$ or two to $3 k$. Moreover, we have $\operatorname{deg}\left(v, B_{2}\right) \geq\left|B_{2}\right|-\beta n$ for $v \in A_{2}, \operatorname{deg}\left(v, A_{2}\right) \geq\left|A_{2}\right|-8 k^{2} \beta n$ for $v \in B_{2}$, and $\left|V_{2}\right| \leq 6 k \beta n$, which implies $\left|A_{2}\right| \geq|A|-\left|V_{1}\right|-\left|V_{2}\right| \geq \frac{n}{2(k+1)}$.

Covering everything and connecting. In this step we first cover $B_{2}$ with copies of $P_{k}^{2}$. Then, using the uncovered vertices in $A_{2}$, we connect all the copies of squares of paths found so far, to get the square of a Hamilton cycle. Observe that after the cleaning steps, $k$ divides $\left|B_{2}\right|$ by (3) and thus Theorem 2.3 implies that a.a.s the random graph $G_{3}\left[B_{2}\right]$ has a $P_{k}^{2}$-factor. We denote the family of such copies of $P_{k}^{2}$ by $\mathcal{F}_{3}$, and observe that (3) implies $\left|\mathcal{F}_{3}\right|=\left|A_{2}\right|-\left|\mathcal{F}_{1}\right|-\left|\mathcal{F}_{2}\right|$.

We let $\mathcal{F}=\mathcal{F}_{1} \cup \mathcal{F}_{2} \cup \mathcal{F}_{3}$ be the family of all the squares of paths that we have constructed and, for each $F \in \mathcal{F}$, denote the end-tuples of $F$ by $\left(y_{F}, x_{F}\right)$ and $\left(u_{F}, w_{F}\right)$. Note that by construction, each pair $x_{F}, y_{F}$ and $u_{F}, w_{F}$ has at least $\left|A_{2}\right|-16 k^{2} \beta n$ common neighbours in $A_{2}$. We now reveal the edges of $G_{4}$ and construct an auxiliary directed graph $\mathcal{T}$ on vertex set $\mathcal{F}$ as follows. Given any two $F, F^{\prime} \in \mathcal{F}$, there is a directed edge $\left(F, F^{\prime}\right)$ if and only if the edge $w_{F} x_{F^{\prime}}$ appears in $G_{4}$. Since all directed edges in $\mathcal{T}$ are revealed with probability $\frac{1}{4} p$ independently of all the others, $\mathcal{T}$ is distributed as $\vec{G}\left(|\mathcal{F}|, \frac{1}{4} p\right)$. Then, as $|\mathcal{F}| \geq \frac{1}{2 k} n$ and $\frac{1}{4} p \geq C_{4} \log |\mathcal{F}| /|\mathcal{F}|$, there a.a.s. is a directed Hamilton $\vec{C}$ cycle in $\mathcal{T}$ by Theorem 2.5.

In order to get the desired square of a Hamilton path, it remains to match the edges $\left(F, F^{\prime}\right)$ of $\vec{C}$ to the vertices $v \in A_{2}$ such that $u_{F}, w_{F}, x_{F^{\prime}}, y_{F^{\prime}}$ are all neighbours of $v$ in the graph $G$. Observe indeed that $|E(\vec{C})|=\left|\mathcal{F}_{1}\right|+\left|\mathcal{F}_{2}\right|+\left|\mathcal{F}_{3}\right|=\left|A_{2}\right|$, the cycle $\vec{C}$ gives a cyclic ordering of the squares of paths in $\mathcal{F}$, and between consecutive copies $\left(F, F^{\prime}\right)$ we have the edge $w_{F} x_{F^{\prime}}$ (by definition of the graph $\mathcal{T}$ ) and the matching will give a unique vertex $v$ incident to $u_{F}, w_{F}, x_{F^{\prime}}$ and $y_{F^{\prime}}$. For that we define the following auxiliary bipartite graph $\mathcal{B}$ with classes $E(\vec{C})$ and $A_{2}$. There is an edge between $\left(F, F^{\prime}\right) \in E(\vec{C})$ and $v \in A_{2}$ if and only if $u_{F}, w_{F}, x_{F^{\prime}}, y_{F^{\prime}}$ are all neighbours of $v$ in the graph $G$. The existence of a perfect matching in $\mathcal{B}$ easily follows from Hall's condition, as the minimum degree of $\mathcal{B}$ is large: by the choice of $\beta$ and the lower bound on $\left|A_{2}\right|$, the degree of $\left(F, F^{\prime}\right) \in E(\vec{C})$ in $\mathcal{B}$ is at least $\left|A_{2}\right|-32 k^{2} \beta n \geq \frac{1}{2}\left|A_{2}\right|$ and the degree of $v \in A_{2}$ in $\mathcal{B}$ is at least $\left|A_{2}\right|-2 \beta n \geq \frac{1}{2}\left|A_{2}\right|$. This finishes the proof.

\section{Proof of Theorem 3.4}

Proof of Theorem 3.4. Let $k \geq 2$ and $0<\beta<\frac{1}{6 k}$. We obtain $d>0$ from Lemma 3.6 with input $k$ and $\beta$, and let $0<\delta^{\prime}<2^{-12} d^{2 k^{2}}$ and $\gamma=\frac{1}{2} d$. Next we obtain $\delta_{0}, \delta, \varepsilon^{\prime}>0$ and $C_{1}$ from Lemma 3.7 with input $k, \delta^{\prime}$, and $d$ such that $\delta^{\prime} \geq \delta_{0}>2 \delta$ ( $\delta$ plays the role of $\delta_{1}$ in the lemma). We additionally assume that $\varepsilon^{\prime}$ is small enough for Lemma 3.8 with input $\frac{1}{2} d$ and also obtain $C_{2}$ from this. Then we let $0<\varepsilon<\frac{1}{8} \varepsilon^{\prime}$. The constant dependencies can be summarised as follows:

$$
\varepsilon \ll \varepsilon^{\prime} \ll \delta \ll \delta_{0} \ll \delta^{\prime} \ll d \ll \beta<\frac{1}{6 k} .
$$

We now apply Lemma 2.8 with input $\varepsilon$ and $t_{0}=\frac{1}{10} d$, and get $T$. Further, let the following parameters be given by Lemma 2.4: $C_{3}$ for input 3 (in place of $s$ ), 2 (in place of $k$ ), and $\delta(k T)^{-6}$ (in place of $\eta$ ); $C_{4}$ for input $2 k, k$, and $\delta(k T)^{-2 k^{2}} ; C_{5}$ for input 4,2 , and $\delta(k T)^{-8}$; and $C_{6}$ for input 1,2 , and $\delta(k T)^{-2}$. Then let $C$ be large enough such that with $p \geq C n^{-(k-1) /(2 k-3)}$ the random graph $G(n, p)$ contains the union $\cup_{i=1}^{6} G_{i}$, where 
$G_{1} \sim G\left(n, C_{1} \cdot 2(k-1) T n^{-(k-1) /(2 k-3)}\right), G_{2} \sim G\left(n, C_{2} \cdot 2(k-1) T n^{-1}\right), G_{3} \sim G\left(n, C_{3} n^{-1}\right)$, $G_{4} \sim G\left(n, C_{4} n^{-(k-1) /(2 k-3)}\right), G_{5} \sim G\left(n, C_{5} n^{-1}\right)$, and $G_{6} \sim G\left(n, C_{6} n^{-1}\right)$.

Let $G$ be an $n$-vertex graph with vertex set $V$ and minimum degree $\delta(G) \geq\left(\frac{1}{k+1}-\gamma\right) n$ that is not $\left(\frac{1}{k+1}, \beta\right)$-stable. We apply the Regularity Lemma 2.8 to $G$ and get a subgraph $G^{\prime}$ of $G$, a constant $t$ with $3<t+1 \leq T$, and an $(\varepsilon, d)$-regular partition $V_{0}^{\prime}, \ldots, V_{t}^{\prime}$ of $V$, satisfying (P1)-(P4). Consider the $(\varepsilon, d)$-reduced graph $R$ for $G$ and observe $\delta(R) \geq$ $\left(\frac{1}{k+1}-2 d\right) t$, as, otherwise, in $G^{\prime}$ there would be a vertex of degree at most $\left(\frac{1}{k+1}-2 d\right) t \frac{n}{t}+\varepsilon n<$ $\left(\frac{1}{k+1}-\gamma\right) n-(d+\varepsilon) n$ in contradiction to (P2). As outlined in Section 3.5, the proof will consist of four steps. We will cover the reduced graph with copies of stars isomorphic to $K_{1, k}$ and $K_{1,1}$, connect those stars with the squares of short paths, cover the exceptional vertices, and finally cover the whole graph with the square of a Hamilton path.

Covering $R$ with stars. We start by covering the vertices of $R$ with vertex-disjoint stars, each with at most $k$ leaves; then we will turn this into a cover with copies of stars isomorphic to $K_{1, k}$ and $K_{1,1}$. We let $M_{1}$ be a largest matching in $R$ and, with Lemma 3.6, we get that $\left|M_{1}\right| \geq\left(\frac{1}{k+1}+2 k d\right) t$ since $G$ is not $\left(\frac{1}{k+1}, \beta\right)$-stable. By the maximality of $M_{1}$, the remaining vertices $X_{1}=V(R) \backslash V\left(M_{1}\right)$ form an independent set in $R$; moreover only one endpoint of each edge in $M_{1}$ can be adjacent to more than one vertex from $X_{1}$ and the endpoints of each edge in $M_{1}$ cannot have different neighbours in $X_{1}$. For each $i=2, \ldots, k$, we greedily pick a maximal matching $M_{i}$ between $X_{i-1}$ and $V\left(M_{1}\right)$ and we set $X_{i}=X_{i-1} \backslash V\left(M_{i}\right)$. Observe that, using the properties coming from the maximality of $M_{1}$ outlined above, the matching $M_{i}$ covers at least $\min \left\{\left|X_{i-1}\right|, \delta(R)\right\}$ vertices of $X_{i-1}$. Since $2\left|M_{1}\right|+(k-1) \delta(R) \geq t$, we have $X_{k}=\varnothing$ and the union $\bigcup_{i=1}^{k} M_{i}$ covers all vertices of $R$ with stars, each isomorphic to $K_{1, i}$ for some $i=1, \ldots, k$. Moreover note that the number of $K_{1, k}$ is at most $\left|M_{k}\right| \leq t-2\left|M_{1}\right|-(k-2) \delta(R) \leq\left(\frac{1}{k+1}-2 k d\right) t$.

For simplicity we only want to work with stars isomorphic to $K_{1, k}$ and $K_{1,1}$. To obtain this, we split each cluster $V$ arbitrarily into $k-1$ parts $V^{1}, \ldots, V^{k-1}$ of the same size, where we move at most $t(k-1)$ vertices to $V_{0}^{\prime}$ for divisibility reasons. Note that from any $(\varepsilon, d)$-regular pair we get $(k-1)^{2}$ pairs that are $(k \varepsilon, d-\varepsilon)$-regular. We denote this new partition by $V_{0}=V_{0}^{\prime}, V_{1}, \ldots, V_{t^{\prime}}$ with $t^{\prime}=t(k-1)$ and denote the reduced graph for this partition by $R^{\prime}$. We now show that we can cover $R^{\prime}$ with copies of stars isomorphic to $K_{1, k}$ and $K_{1,1}$. Any copy of $K_{1,1}$ or $K_{1, k}$ in $R$ immediately gives $k-1$ copies of $K_{1,1}$ or $K_{1, k}$ in $R^{\prime}$, respectively. Moreover given any copy $V, U_{1}, \ldots, U_{i}$ of $K_{1, i}$ in $R$, with $V$ being the center cluster and $2 \leq i \leq k-1$, we find $i-1$ copies of $K_{1, k}$ and $k-i$ copies of $K_{1,1}$ in $R^{\prime}$ in the following way. For each $j=1, \ldots, i-1$, the clusters $V^{j}, U_{j}^{1}, \ldots, U_{j}^{k-1}, U_{i}^{j}$ give a copy of $K_{1, k}$ in $R^{\prime}$, and for each $j=i, \ldots, k-1$ the clusters $V^{j}, U_{i}^{j}$ give a copy of $K_{1,1}$ in $R^{\prime}$. Therefore, we have covered the vertices of $R^{\prime}$ with a collection $\mathcal{K}$ of copies of $K_{1, k}$ and $K_{1,1}$. We remark that we can still upper bound the number of copies of $K_{1, k}$ in $\mathcal{K}$ as follows. Since each copy of $K_{1, i}$ in the original cover gives $i-1$ copies of $K_{1, k}$ in $\mathcal{K}$, we get the largest number of copies of $K_{1, k}$ in $\mathcal{K}$ when the total number of stars in the original cover is minimal. The original cover of $R$ had at most $\left(\frac{1}{k+1}-2 k d\right) t$ copies of $K_{1, k}$, and the remaining $t-(k+1)\left(\frac{1}{k+1}-2 k d\right) t=2 k(k+1) d t$ vertices can give at most $\frac{2 k(k+1) d t}{k}=2(k+1) d t$ copies of $K_{1, k-1}$. Therefore the collection $\mathcal{K}$ has at most $(k-1)\left(\frac{1}{k+1}-2 k d\right) t+(k-2) 2(k+1) d t \leq\left(\frac{1}{k+1}-4 k d\right) t^{\prime}$ copies of $K_{1, k}$. We let $n_{0}=\left|V_{1}\right|=$ $\left\lfloor\left|V_{1}^{\prime}\right| /(k-1)\right\rfloor$ be the size of the clusters in $R^{\prime}$ and observe that $(1-2 \varepsilon) n / t^{\prime} \leq n_{0} \leq n / t^{\prime}$.

For convenience we relabel the clusters as follows. We let $\mathcal{I} \subseteq\left[t^{\prime}\right]$ be the set of indices of those clusters of $R^{\prime}$ that are the centre cluster in a copy of $K_{1, k}$ in $\mathcal{K}$, and for $i \in \mathcal{I}$ we denote by $U_{i, 1}, \ldots, U_{i, k}$ the clusters of $R^{\prime}$ that, together with $V_{i}$, create a copy of $K_{1, k}$ in $\mathcal{K}$. Then we let $\mathcal{J} \subseteq\left[t^{\prime}\right]$ be any set of indices with the following property: each index in 
$\mathcal{J}$ corresponds to a cluster of a copy of $K_{1,1}$ in $\mathcal{K}$, and for each copy of $K_{1,1}$ in $\mathcal{K}$, exactly one of its clusters has its index in $\mathcal{J}$. Moreover, for each $i \in \mathcal{J}$, we let $U_{i, 1}$ be the cluster of $R^{\prime}$ that, together with $V_{i}$, creates a copy of $K_{1,1}$ in $\mathcal{K}$.

We would like to apply Lemma 3.7 and Lemma 3.8 to the clusters corresponding to the copies of $K_{1, k}$ and $K_{1,1}$, respectively. However we first need to make each regular pair super-regular and unbalance some of the clusters to allow an application of Lemma 3.7. For that we arbitrarily move $\delta n_{0}$ vertices from each cluster $U_{i, j}$ with $i \in \mathcal{I}$ and $j=1, \ldots, k$ to $V_{0}$. Next we repeatedly use Lemma 2.7 and move at most $k^{2} \varepsilon n_{0}$ vertices from each cluster to $V_{0}$, to ensure that all edges of $R^{\prime}$ within a copy of $K_{1, k}$ or $K_{1,1}$ from $\mathcal{K}$ are $(2 k \varepsilon, d-4 k \varepsilon)$-super-regular. When doing this we can ensure that for each $i \in \mathcal{I}$ all clusters $V_{i}$ have the same size, and that for $j=1, \ldots, k$ all clusters $U_{i, j}$ have the same size, except the cluster $U_{i, 1}$ that has two more vertices than the other $U_{i, j}$ 's. Moreover, we can ensure that for $i \in \mathcal{I}$ we have $\left|V_{i}\right|-4-\left|U_{i, k}\right| \equiv 0(\bmod 6 k-2)$, again in view of a later application of Lemma 3.7. Note that at this point we have $\left|V_{0}\right| \leq \varepsilon n+t(k-1)+t^{\prime} \delta n_{0}+t^{\prime} k^{2} \varepsilon n_{0} \leq 2 \delta n$.

Connecting the stars. In this step, we fix an arbitrary cyclic ordering of the copies of $K_{1, k}$ and $K_{1,1}$, and we connect each consecutive pair using the square of a short path. For the rest of the proof, we will refer to these (squares of) short paths as the connecting paths. We first explain the connection between two copies of $K_{1, k}$ and assume without loss of generality that $1,2 \in \mathcal{I}$; when at least one of the copy is $K_{1,1}$, the connection is similar and will be explained later. We use the square of a path on six vertices with end-tuples within $V_{1}$ and $V_{2}$, such that, again in view of Lemma 3.7, the end-tuples have many common neighbours into the other clusters $U_{1, j}$ and $U_{2, j}$ for $j=1, \ldots, k$, respectively. Recall that both $U_{1,1}$ and $U_{2,1}$ contain two more vertices than the other leaf clusters, one of which will be used for this connection.

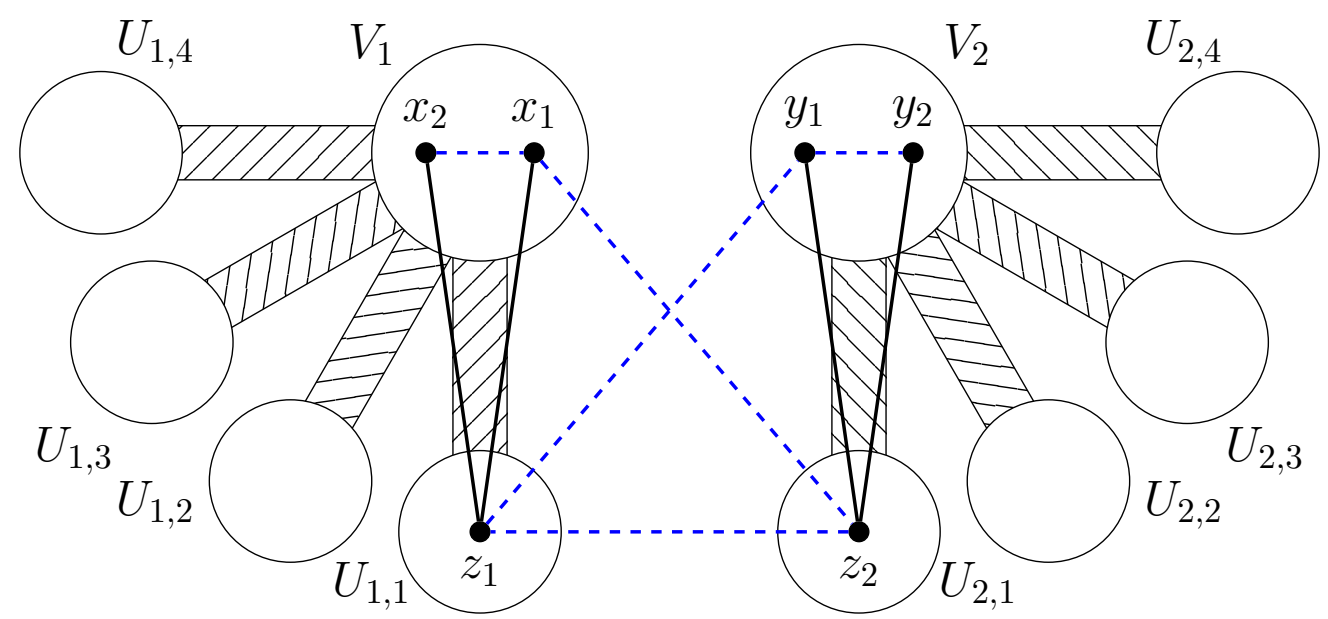

Figure 3. Construction of the square of a path with end-tuples $\left(x_{1}, x_{2}\right)$ and $\left(y_{1}, y_{2}\right)$ that connects two copies of $K_{1, k}$ in the cluster graph $R^{\prime}$. The dashed blue edges come from the random graph and the black edges from the deterministic graph.

We want to find $x_{1}, x_{2} \in V_{1}, z_{2} \in U_{2,1}, z_{1} \in U_{1,1}$, and $y_{1}, y_{2} \in V_{2}$ such that the following holds (see Figure 3):

(A1) the tuples $\left(x_{1}, x_{2}\right)$ and $\left(y_{1}, y_{2}\right)$ have at least $\frac{3}{4} d^{2} n_{0}$ common neighbours in each of $U_{1,1}, \ldots, U_{1, k}$ and $U_{2,1}, \ldots, U_{2, k}$ in the graph $G$, respectively;

(A2) $x_{1} z_{1}, x_{2} z_{1}, y_{1} z_{2}$, and $y_{2} z_{2}$ are edges of $G$;

(A3) $x_{2}, x_{1}, z_{2}, z_{1}, y_{1}, y_{2}$ is a path in $G_{3}$. 
For that we use Lemma 2.4 on the following collection $H$ of tuples. We pick subsets $X$ in $V_{1}, Y$ in $V_{2}, Z_{1}$ in $U_{1,1}$, and $Z_{2}$ in $U_{1,2}$, all of size $n_{0} / 3$, and we let $H$ the set of those tuples $\left(x_{2}, x_{1}, z_{2}, z_{1}, y_{1}, y_{2}\right) \in X \times X \times Z_{2} \times Z_{1} \times Y \times Y$ which satisfy the properties (A1) and (A2). Note that $H$ contains enough tuples for an application of Lemma 2.4 as, from regularity, we get that for all but at most $2 \varepsilon n_{0}$ vertices $x_{1} \in X$, all but $4 k \varepsilon n_{0}$ vertices $x_{2} \in X$ have at least $\frac{3}{4} d^{2} n_{0}$ common neighbours in $U_{1,1}, \ldots, U_{1, k}$ and at least $\frac{1}{4} d^{2} n_{0}$ common neighbours in $Z_{1}$ with respect to $G$ and similarly for $Y$ with $U_{2,1}, \ldots, U_{2, k}$ and $Z_{2}$. Therefore, $H$ has size at least $d^{4} 2^{-12} n_{0}^{6} \geq \delta(k T)^{-6} n^{6}$. Now we reveal the edges of $G_{3}\left[X \cup Z_{2} \cup Z_{1} \cup Y\right]$ and with Lemma 2.4 and given the choice of $C_{3}$, we a.a.s. find a tuple $\left(x_{2}, x_{1}, z_{2}, z_{1}, y_{1}, y_{2}\right) \in H$ satisfying the property (A3) as well. This will give the desired connecting square of a path. We then remove the vertices $z_{1}$ and $z_{2}$ from $U_{1,1}$ and $U_{2,1}$, respectively.

Given an arbitrary cycling ordering of the indices from $\mathcal{I}$ and $\mathcal{J}$, we use this construction to connect all neighbouring pairs $(i, j)$. If $i, j \in \mathcal{I}$ we proceed as described above for the pair $(1,2)$; if $i \in \mathcal{J}$, we let $U_{i, 1}$ take the role of all $U_{1,1}, \ldots, U_{1, k}$; and if $j \in \mathcal{J}$, we let $U_{j, 1}$ take the role of $V_{2}$ and $V_{j}$ the role of all $U_{2,1}, \ldots, U_{2, k}$. As we pick sets $X, Y$, $Z_{1}$ and $Z_{2}$ of size $n_{0} / 3$ and each cluster is involved in at most two distinct connections, we can choose disjoint sets for each connection and avoid clashes. Therefore each edge of $G_{3}$ is revealed at most once and, as there are at most $t^{\prime}$ connections, we a.a.s. get all the desired edges of $G_{3}$. Observe that the choices done so far are needed for a later application of Lemma 3.7 and Lemma 3.8. Indeed for $i \in \mathcal{I}$ there are two end-tuples of connecting paths in $V_{i}$, and for $i \in \mathcal{J}$ there is one end-tuple of a connecting path in each of $V_{i}$ and $U_{i, 1}$; moreover, for $i \in \mathcal{I}$, since $U_{i, 1}$ is involved in exactly two connections, during this construction we removed exactly two vertices from $U_{i, 1}$, and now all $U_{i, j}$ 's have the same size for $j=1, \ldots, k$.

Covering $V_{0}$. In the next step we cover all vertices of $V_{0}$ by extending the connecting paths that we have already constructed and we recall that $\left|V_{0}\right| \leq 2 \delta n$. It is crucial for the rest of the argument (in particular for the applications of Lemma 3.7 and 3.8) that the conditions on the relation between the sizes of the clusters are still satisfied and that the end-tuples remain in the same clusters, i.e. if we extend the square of a path with one end-tuple in a cluster $V_{i}$, then the extended path needs to have the new end-tuple in the same cluster $V_{i}$. We will again make use of Lemma 2.4, with a suitable collection of tuples $H$. Before giving a precise description, we refer to Figure 4 and illustrate the extension when $k=3$ and we want to cover a vertex $v \in V_{0}$ by extending the square of a connecting path with end-tuple $\left(x_{1}, x_{2}\right)$ in the centre cluster $V_{i}$ of a copy of $K_{1,3}$. We will find 24 additional vertices as drawn in Figure 4, where we stress the following conditions. The vertices $v_{3,1}, \ldots, v_{3,6}$ are all neighbours of $v$ in $G$ (which will be guaranteed by the minimum degree condition), the blue edges are random edge (which will be guaranteed by Lemma 2.4), while the black edges are from the graph $G$ (which will be guaranteed by regularity). Thus we extend the connecting path into the square of a path with end-tuples $\left(x_{2}, x_{1}\right)$ and $\left(x_{2}^{\prime}, x_{1}^{\prime}\right)$ still in $V_{i}$. Moreover, since six new vertices have been covered from each cluster, the relation between their sizes still holds.

We will now give the details of these constructions and we start by defining the collections of tuples we will use for the applications of Lemma 2.4. For $i \in \mathcal{I}$, we let $H_{1, i}$ be the set of those tuples in $\prod_{j=1}^{k}\left(U_{i, j}^{k} \times U_{i, j}^{k}\right)$ such that the $2 k^{2}$ vertices in each tuple have at least $\frac{1}{2} d^{2 k^{2}} n_{0}$ common neighbours in $V_{i}$ in $G$. Then we let $H_{1}=\bigcup_{i \in \mathcal{I}} H_{1, i}$. Similarly, for $j \in \mathcal{J}$, we let $H_{2, j}$ be the set of those tuples in $V_{j}^{8} \cup U_{j, 1}^{8}$ such that the 8 vertices in each tuple have at least $\frac{1}{2} d^{8} n_{0}$ common neighbours in the other set in $G$. Then we let $H_{2}=\bigcup_{j \in \mathcal{J}} H_{2, j}$. Moreover, for $i \in \mathcal{I}$, we let $H_{3, i}$ be the set of those tuples in $V_{i}^{2}$ such that the 2 vertices in each tuple have at least $\frac{3}{4} d^{2} n_{0}$ common neighbours in each of the sets 

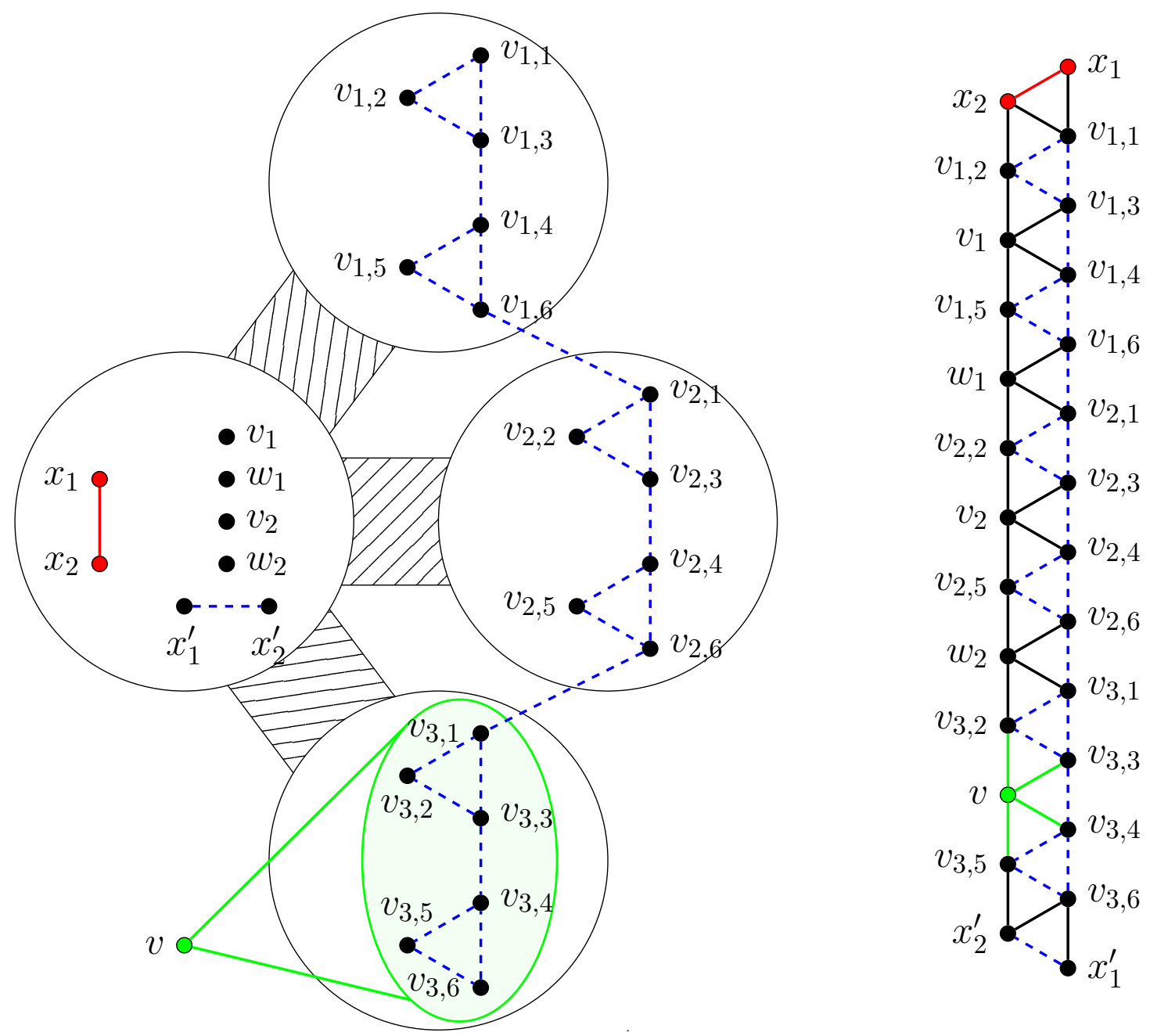

Figure 4. Covering one vertex $v \in V_{0}$ by the square of a path for a copy of $K_{1, k}$ in $\mathcal{K}$ for $k=3$. We keep the position of the end-tuples in $V_{i}$ and the sizes of the clusters balanced. The edges within the clusters (dashed blue) come from the random graph and the edges between the clusters (black) come from regularity.

$U_{i, j}$ for $j=1, \ldots, k$. For $j \in \mathcal{J}$, we let $H_{3, j}$ be the set of those tuples in $V_{j}^{2} \cup U_{j, 1}^{2}$ such that the 2 vertices in each tuple have at least $\frac{3}{4} d^{2} n_{0}$ common neighbours in the other set in $G$. Then we let $H_{3}=\bigcup_{i \in \mathcal{I}} H_{3, i} \cup \bigcup_{j \in \mathcal{J}} H_{3, j}$. With the constants specified in the beginning of the proof for obtaining $C_{4}, C_{5}$, and $C_{6}$, we apply Lemma 2.4 to $H_{1}$ with the random graph $G_{4}$, to $H_{2}$ with $G_{5}$, and to $H_{3}$ with $G_{6}$. Given the choice of the constant $C$ done at the beginning, we can a.a.s. assume that

$G_{4}, G_{5}$ and $G_{6}$ are all in the good event of Lemma 2.4 for the application above.

Now we explain how we cover the vertices of $V_{0}$ and we show that suitable subsets of $H_{1}, H_{2}$ and $H_{3}$ are large enough for Lemma 2.4, i.e. larger than $\delta(k T)^{-s k} n^{s k}$ where $s k=2,8,2 k^{2}$, respectively.

Given a vertex $v \in V_{0}$, we insist that the neighbours of $v$ that we use to cover $v$ do not come from any of the centre clusters $V_{i}$ with $i \in \mathcal{I}$, because in this case we could not ensure to use the same number of vertices from each cluster in the copy of $K_{1, k}$ and we would unbalance the star, creating issues for a later application of Lemma 3.7. Observe that, as we have $\left|V_{0} \cup\left(\bigcup_{i \in \mathcal{I}} V_{i}\right)\right| \leq 2 \delta n+\left(\frac{1}{k+1}-4 k d\right) t^{\prime} n_{0} \leq\left(\frac{1}{k+1}-\gamma\right) n-2 d n$, every vertex in $G$ has at least $2 d n$ neighbours outside of $V_{0} \cup\left(\cup_{i \in \mathcal{I}} V_{i}\right)$ in the graph $G$. Moreover, for 
each vertex $v \in V_{0}$, we will use at most $\max \{2 k(k+1), 22\} \leq 6 k^{2}$ vertices outside of $V_{0}$. During the process of covering $V_{0}$, we let $V^{\star} \subseteq V_{0}$ be the set of vertices of $V_{0}$ already covered and $W$ be the set of vertices outside of $V_{0}$ that we already used to cover $V^{*}$; at the beginning $V^{*}=\varnothing$ and $W=\varnothing$. Note that we have $|W| \leq 6 k^{2}\left|V^{*}\right| \leq 12 k^{2} \delta n$. Then we let $\mathcal{T} \subseteq \mathcal{I} \cup \mathcal{J}$ be the set of those indices $i \in \mathcal{I} \cup \mathcal{J}$ such that $U_{i, 1}$ intersects $W$ in at least $\sqrt{\delta} n_{0}$ vertices and note that the bound on $|W|$ implies that $|\mathcal{T}| \leq 12 k^{2} \sqrt{\delta} t^{\prime}$. We remark that, since throughout the process we always cover the same number of vertices in each cluster of a copy of $K_{1,1}$ or $K_{1, k}$, if $i \notin \mathcal{T}$, then $W$ interesect each cluster of the copy corresponding to the index $i$ in less then $\sqrt{\delta} n_{0}$ vertices. Moreover, notice that as each $v \in V_{0}$ has at least $2 d n$ neighbours outside of $V_{0} \cup\left(\cup_{i \in \mathcal{I}} V_{i}\right)$, there are at least $d t^{\prime}$ clusters that are not the centre cluster of a copy of $K_{1, k}$ and such that $v$ has at least $d n_{0}$ neighbours in it. Therefore, for every $v \in V_{0} \backslash V^{*}$ there exists $i(v) \in(\mathcal{I} \cup \mathcal{J}) \backslash \mathcal{T}$ and some $j(v)$ such that $v$ has at least $d n_{0}$ neighbours in $U_{i(v), j(v)} \backslash W$ with respect to $G$.

Fix any $v \in V_{0} \backslash V^{*}$ and let $i=i(v)$. We start discussing the case $i \in \mathcal{I}$; the case $i \in \mathcal{J}$ is conceptually simpler and will be treated afterwards. Without loos of generality we can assume $j(v)=k$. Let $\left(x_{1}, x_{2}\right)$ and $\left(y_{1}, y_{2}\right)$ be the end-tuples in $V_{i}$ of the connecting paths found in the previous step, and recall that $x_{1}$ and $x_{2}$ have at least $\frac{3}{4} d^{2} n_{0}$ common neighbours in $U_{i, j}$ for each $j=1, \ldots, k$. We will extend the square of the connecting path ending in $\left(x_{1}, x_{2}\right)$, without using neither $y_{1}$ nor $y_{2}$. Moreover, we will make sure that the new end-tuple $\left(x_{2}^{\prime}, x_{1}^{\prime}\right)$ will still belong to $V_{i}$, and that $x_{1}$ and $x_{2}$ will have at least $\frac{3}{4} d^{2} n_{0}$ common neighbours in $U_{i, j}$ for each $j=1, \ldots, k$.

Let $Z_{1}$ be the set $\left(N_{G}\left(x_{1}, U_{i, 1}\right) \cap N_{G}\left(x_{2}, U_{i, 1}\right)\right) \backslash\left(W \cup\left\{y_{1}, y_{2}\right\}\right)$, let $Z_{j}$ be the set $U_{i, j} \backslash W$ for $j=2, \ldots, k-1$, and let $Z_{k}$ be the set $N_{G}\left(v, U_{i, k}\right) \backslash W$. Observe that $\left|Z_{1}\right| \geq \frac{1}{2} d^{2} n_{0}$ since $x_{1}$ and $x_{2}$ have at least $\frac{3}{4} d^{2} n_{0}$ common neighbours in $U_{i, 1},\left|Z_{j}\right| \geq(1-\sqrt{\delta}) n_{0} \geq d n_{0}$ for $j=2, \ldots, k-1$, and $\left|Z_{k}\right| \geq d n_{0}$ by the choice of $i=i(v)$. Using the regularity properties, analogously as above, it is easy to see that there are at least $\delta(k T)^{-2 k^{2}} n^{2 k^{2}}$ tuples in $\prod_{j=1}^{k}\left(Z_{j} \times Z_{j}\right)$ such that the $2 k^{2}$ vertices in each tuple have at least $\frac{1}{2} d^{2 k^{2}} n_{0}$ common neighbours in $V_{i}$ in $G$. These tuples are in $H_{1, i} \subseteq H_{1}$ as well and thus (4) guarantees that we find one of such tuples $\left(v_{j, j^{\prime}}: 1 \leq j \leq k, 1 \leq j^{\prime} \leq 2 k\right)$ in $\prod_{j=1}^{k}\left(Z_{j} \times Z_{j}\right)$ where $v_{j, j^{\prime}} \in Z_{j}$, such that in $G_{4}$ we have the square of a path on $v_{j, 1}, \ldots, v_{j, k}$, the square of a path on $v_{j, k+1}, \ldots, v_{j, 2 k}$, and the edges $v_{j, k} v_{j, k+1}$ and $v_{j, 2 k} v_{j+1,1}$.

Let $Z \subseteq V_{i} \backslash\left(W \cup\left\{y_{1}, y_{2}\right\}\right)$ be the common neighbourhood of the vertices $v_{j, j^{\prime}}$ with $1 \leq j \leq k$ and $1 \leq j^{\prime} \leq 2 k$ in $V_{i}$, and observe $|Z| \geq \frac{1}{4} d^{2 k^{2}} n_{0}$. Again using regularity, there are at least $\delta(k T)^{-2} n^{2}$ tuples in $Z^{2} \cap H_{3}$, and thus (4) guarantees that there is a tuple $\left(x_{1}^{\prime}, x_{2}^{\prime}\right) \in Z^{2} \cap H_{3}$ such that $x_{1}^{\prime} x_{2}^{\prime}$ is an edge of $G_{6}$ and $x_{1}^{\prime}$ and $x_{2}^{\prime}$ have at least $\frac{3}{4} d^{2} n_{0}$ common neighbours in each $U_{i, j}$ for $j=1, \ldots, k$. We then greedily pick additional $2(k-1)$ vertices $v_{1}, w_{1}, \ldots, v_{k-1}, w_{k-1}$ in $Z$ and we claim that

$$
\begin{aligned}
x_{1}, x_{2}, v_{1,1}, \ldots, v_{1, k}, v_{1}, v_{1, k+1}, \ldots, v_{1,2 k}, w_{1}, \\
v_{2,1}, \ldots, v_{2, k}, v_{2}, \ldots, w_{k-1}, v_{k, 1}, \ldots, v_{k, k}, v, v_{k, k+1}, \ldots, v_{k, 2 k}, x_{1}^{\prime}, x_{2}^{\prime}
\end{aligned}
$$

is the square of a path with end-tuples $\left(x_{2}, x_{1}\right)$ and $\left(x_{1}^{\prime}, x_{2}^{\prime}\right)$ that contains $v$. Indeed, $x_{1}$ and $x_{2}$ are common neighbours of $v_{1,1}$ and $v_{1,2}$, while $x_{1}^{\prime}$ and $x_{2}^{\prime}$ are common neighbours of $v_{k, 2 k-1}$ and $v_{k, 2 k}$; moreover the vertex $v$ is a common neighbour of $v_{k, k-1}, v_{k, k}, v_{k, k+1}$ and $v_{k, k+2}$, the vertex $v_{j}$ is a common neighbour of $v_{j, k-1}, v_{j, k}, v_{j, k+1}$ and $v_{j, k+2}$, and the vertex $w_{j}$ is a common neighbour of $v_{j, 2 k-1}, v_{j, 2 k}, v_{j+1,1}$ and $v_{j+1,2}$. This fills the gaps left after the initial construction above where we used Lemma 2.4 with the graph $G_{4}$. We now add $v$ to $V^{*}$ and all the other used vertices $v_{j, j^{\prime}}$ for $1 \leq j \leq k$ and $1 \leq j^{\prime} \leq 2 k$, and $x_{i}, w_{i}$ for $1 \leq i \leq k-1$ to $W$, with the exception of $x_{1}^{\prime}$ and $x_{2}^{\prime}$ as $\left(x_{2}^{\prime}, x_{1}^{\prime}\right)$ is the end-tuple of the 
extended square of a path. Observe that we used $2 k(k+1)$ vertices to cover $v$ and that we covered exactly $2 k$ vertices from each cluster of the copy of $K_{1, k}$.

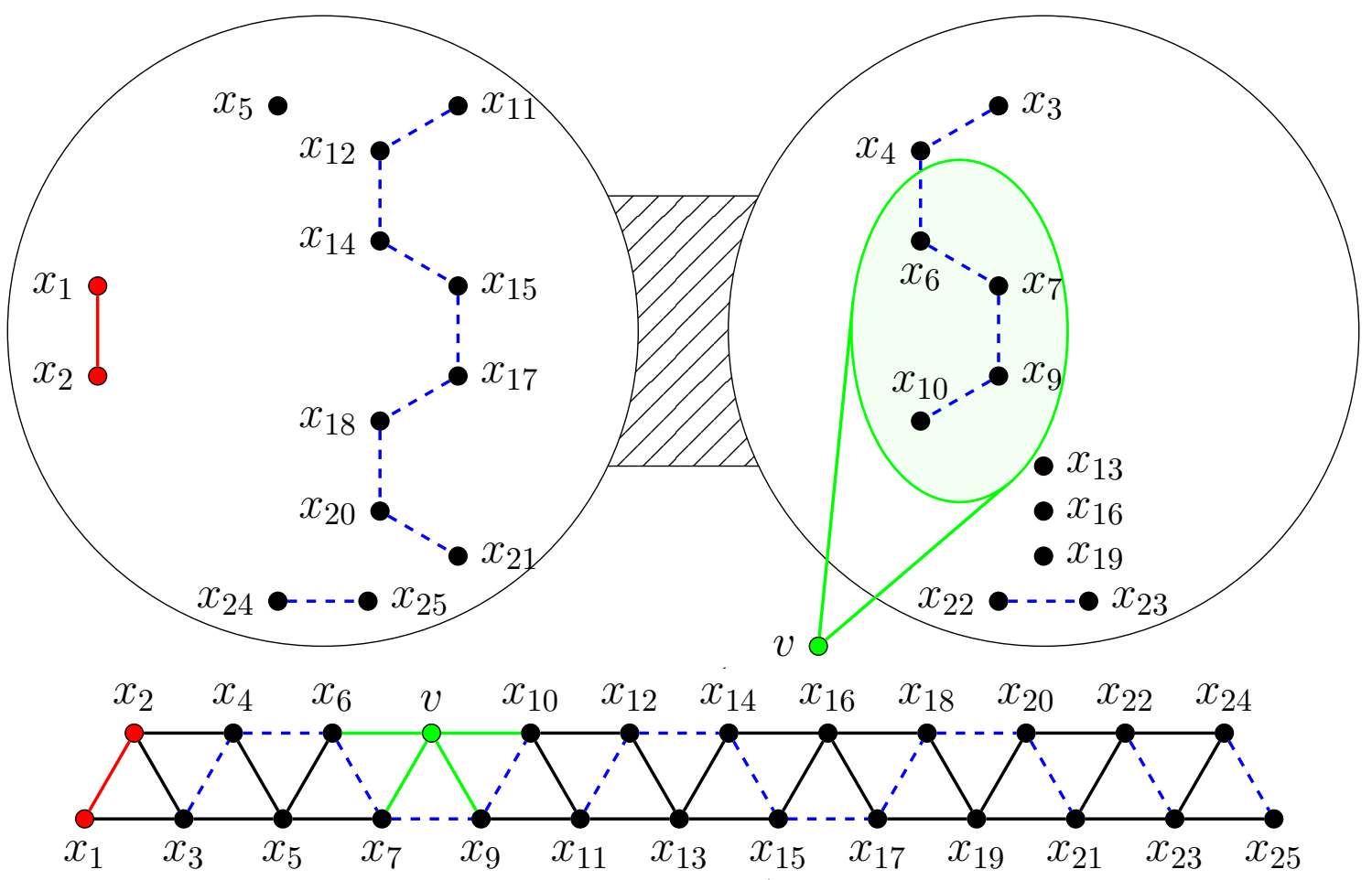

Figure 5. Covering one vertex $v \in V_{0}$ by the square of a path for a copy of $K_{1,1}$ in $\mathcal{K}$. We keep the position of the end-tuples in $V_{i}$ and the sizes of the clusters balanced. The edges within the clusters (dashed blue) come from the random graph and the edges between the clusters (green and black) come from regularity.

Now we move to the construction for the case $i=i(v) \in \mathcal{J}$. We will use always the same construction regardless of the value of $k$ as illustrated in Figure 5 in a similar way as Figure 4 earlier. By assumption we have $j(v)=1$ and thus $\left|N_{G}\left(v, U_{i, 1} \backslash W\right)\right| \geq d n_{0}$. Let $\left(x_{1}, x_{2}\right) \in V_{i}^{2}$ and $\left(y_{1}, y_{2}\right) \in U_{i, 1}^{2}$ be the end-tuples of the connecting paths found in the previous step, and recall that $x_{1}$ and $x_{2}$ have at least $\frac{3}{4} d^{2} n_{0}$ common neighbours in $U_{i, 1}$. We will extend the square of the connecting path ending in $\left(x_{1}, x_{2}\right)$, without using neither $y_{1}$ nor $y_{2}$, by constructing the square of a path on pairwise distinct vertices $x_{1}, x_{2}, \ldots, x_{24}, x_{25}$, where $x_{8}=v$. Moreover, we will make sure that the new end-tuple $\left(x_{24}, x_{25}\right)$ will still belong to $V_{i}$, and that $x_{24}$ and $x_{25}$ will have at least $\frac{3}{4} d^{2} n_{0}$ common neighbours in $U_{i, 1}$. Let $Z_{3}=Z_{4}$ be the common neighbourhood of $x_{1}$ and $x_{2}$ in $U_{i, 1}$ । $\left(W \cup\left\{y_{1}, y_{2}\right\}\right)$ and $Z_{6}=Z_{7}=Z_{9}=Z_{10}$ be the neighbourhood of $v$ in $U_{i, 1} \backslash\left(W \cup\left\{y_{1}, y_{2}\right\}\right)$, and note that $\left|Z_{3}\right| \geq \frac{1}{2} d^{2} n_{0}$ and $\left|Z_{6}\right| \geq d n_{0}-2$. Using regularity, it is easy to see that for at least $\delta(k T)^{-8} n^{8}$ tuples in $Z_{3} \times Z_{4} \times Z_{6} \times Z_{7} \times Z_{9} \times Z_{10} \times U_{i, 1} \times U_{i, 1}$ the common neighbourhood of their vertices in $V_{i}$ has size at least $\frac{1}{2} d^{8} n_{0}$ (where we added the set $U_{i, 1}$ twice only for the following application of Lemma 2.4). Thus (4) guarantees we find a path in $G_{5}$ on six vertices $x_{3}, x_{4}, x_{6}, x_{7}, x_{9}, x_{10}$ (we ignore the two vertices in $U_{i, 1}$ ) with $x_{j} \in Z_{j}$ and such that the set $Z$ of the common neighbours of $x_{3}, x_{4}, x_{6}, x_{7}, x_{9}, x_{10}$ in $V_{i} \backslash\left(W \cup\left\{x_{1}, x_{2}\right\}\right)$ has size at least $|Z| \geq \frac{1}{4} d^{8} n_{0}$. We let $x_{5}$ be any vertex of $Z$. Again using regularity, there are at least $\delta(k T)^{-8} n^{8}$ tuples in $\left(Z \backslash\left\{x_{5}\right\}\right)^{8}$ such that the eight vertices in the tuple have at least $\frac{1}{2} d^{8} n_{0}$ common neighbours in $U_{i, 1}$. These tuples are in $H_{2}$ as well and thus (4) guarantees we find in $G_{5}$ a path on eight vertices $x_{11}, x_{12}, x_{14}, x_{15}, x_{17}, x_{18}, x_{20}, x_{21}$ such that these eight vertices belong to $Z \backslash\left\{x_{5}\right\}$ and the 
set $Z^{\prime}$ of their common neighbours in $U_{i, 1} \backslash\left(W \cup\left\{y_{1}, y_{2}, x_{3}, x_{4}, x_{5}, x_{6}, x_{7}, x_{9}, x_{10}\right\}\right)$ has size $\left|Z^{\prime}\right| \geq \frac{1}{4} d^{8} n_{0}$. We let $x_{13}, x_{16}$, and $x_{19}$ be any three vertices of $Z^{\prime}$. Again with (4), we find an edge $x_{22} x_{23}$ of $G_{6}$ such that $x_{22}, x_{23} \in Z^{\prime} \backslash\left\{x_{13}, x_{16}, x_{19}\right\}$ and their common neighbourhood $Z^{\prime \prime}$ in $V_{i} \backslash\left(W \cup\left\{y_{1}, y_{2}, x_{1}, x_{2}, x_{5}, x_{11}, x_{12}, x_{14}, x_{15}, x_{17}, x_{18}, x_{20}, x_{21}\right\}\right)$ has size at least $\frac{1}{2} d^{2} n_{0}$. With another application of (4) we find an edge $x_{24} x_{25}$ of $G_{6}$ such that $x_{24}, x_{25} \in Z^{\prime \prime}$ and they have at least $\frac{3}{4} d^{2} n_{0}$ common neighbours in $U_{i, 1}$. This gives the square of a path on the vertices $x_{1}, \ldots, x_{7}, v, x_{9}, \ldots, x_{25}$. We add $v$ to $V^{*}$ and $x_{1}, \ldots, x_{7}, x_{9}, \ldots, x_{23}$ to $W$; note we do not remove of $x_{24}$ and $x_{25}$ as $\left(x_{24}, x_{25}\right)$ is the end-tuple of the extended square of a path. Note that we used 22 vertices to cover $v$ and that we covered exactly 11 vertices from each cluster $V_{i}$ and $U_{i, 1}$.

We keep covering the vertices of $V_{0}$ in this way until $V^{*}=V_{0}$, then we remove the vertices in $W$ from the clusters.

Completing the square of the Hamilton cycle. Before finishing the proof, we summarise what we have done so far. We have covered all vertices of $G$, except those that are still in the clusters of a copy of $K_{1,1}$ or $K_{1, k}$, with the squares of short paths with the following properties. Their end-tuples belong to some $V_{i}$ with $i \in \mathcal{I}$, or some $V_{j}$ or some $U_{j, 1}$ with $j \in \mathcal{J}$. Moreover, for each $i \in \mathcal{I}$, the cluster $V_{i}$ contains exactly two of such tuples, say $\left(x_{1}, x_{2}\right)$ and $\left(y_{1}, y_{2}\right)$, such that $x_{1}$ and $x_{2}$ have at least $\frac{3}{4} d^{2} n_{0}-\sqrt{\delta} n_{0} \geq \frac{1}{2} d^{2} n_{0}$ common neighbours in $U_{i, j}$ for $j=1, \ldots, k$, and the same holds for $y_{1}$ and $y_{2}$. Additionally, for each $j \in \mathcal{J}$, the clusters $V_{j}$ and $U_{j, 1}$ contain exactly one such tuple each, say $\left(x_{1}, x_{2}\right)$ and $\left(y_{1}, y_{2}\right)$ respectively, such that $x_{1}$ and $x_{2}$ have at least $\frac{1}{2} d^{2} n_{0}$ common neighbours in $U_{j, 1}$, and $y_{1}$ and $y_{2}$ have at least $\frac{1}{2} d^{2} n_{0}$ common neighbours in $V_{j}$. Therefore, it remains to cover the clusters by extending the squares of paths we already have.

Let $i \in \mathcal{I}$. Note that we still have that $\left|U_{i, 1}\right|=\cdots=\left|U_{i, k}\right|$ and $\left|V_{i}\right|-4-\left|U_{i, k}\right| \equiv 0(\bmod 6 k-2)$ for $i \in \mathcal{I}$. Recall that we removed $\delta n_{0}$ vertices from each $U_{i, j}$ at the beginning. Moreover, while making the regular pairs super-regular and during the previous step, we removed the same number of vertices from each cluster of a copy of $K_{1, k}$, and at most $2 \sqrt{\delta} n_{0}$ vertices from each. Therefore, using that $2 \delta<\delta_{0}$, we get $\left|U_{i, j}\right| \geq\left(1-\delta_{0}\right)\left|V_{i}\right|$ for $j=1, \ldots, k$. Note that also $\left|U_{i, k}\right| \leq(1-\delta)\left|V_{i}\right|$ still holds. Now let $\left(x_{1}, x_{2}\right)$ and $\left(y_{1}, y_{2}\right)$ be the two end-tuples in $V_{i}$ of connecting paths and recall they both have at least $\frac{1}{2} d^{2} n_{0}$ common neighbours in $U_{i, j}$ for each $j=1, \ldots, k$. We apply Lemma 3.7 with the random graph $G_{1}$ to $V_{i}, U_{i, 1}, \ldots, U_{i, k}$ and get the square of a path with end-tuples $\left(x_{2}, x_{1}\right)$ and $\left(y_{2}, y_{1}\right)$, covering all vertices in $V_{i} \cup U_{i, 1} \cup \ldots, U_{i, k}$.

For $i \in \mathcal{J}$ we proceed similarly. We have $\left|V_{i}\right|=\left|U_{i, 1}\right|$ and two end-tuples $\left(x_{1}, x_{2}\right)$ in $V_{i}$ and $\left(y_{1}, y_{2}\right)$ in $U_{i, 1}$ of connecting paths. Since $x_{1}$ and $x_{2}$ have at least $\frac{1}{2} d^{2} n_{0}$ common neighbours in $U_{i, 1}$, and $y_{1}$ and $y_{2}$ have at least $\frac{1}{2} d^{2} n_{0}$ common neighbours in $V_{i}$, we can apply Lemma 3.8 with the random graph $G_{2}$ to $V_{i}, U_{i, 1}$, and get the square of a path with end-tuples $\left(x_{2}, x_{1}\right)$ and $\left(y_{2}, y_{1}\right)$, covering all vertices in $V_{i} \cup U_{i, 1}$.

This completes the square of a Hamilton cycle covering all vertices of $G$, and finishes the proof.

\section{REgUlarity IN AUXILIARY GRAPHS}

The aim of this section is to prove the main technical lemma behind Lemma 3.7, whose proof is provided in Section 7. In Lemma 3.7, we have to find the square of a Hamilton path (subject to additional conditions), where we can use both deterministic and random edges. Here we look at the edges coming from the random graph and show that we can find many disjoint copies of the square of a short path in the random graph, with some additional properties with respect to the deterministic graph, which guarantee we 
can then nicely connect them and build the desired structure. We state a more general version of the result we need for Lemma 3.7, as we believe it might be of independent interest and helpful in other problems of similar flavour. Before stating the lemma we introduce some definitions.

Consider any graph $H$ and set $h=|V(H)|$. Let $V, U_{1}, \ldots, U_{h}$ be disjoint sets of vertices and $G$ be a graph on vertex set $V \cup U_{1} \cup \cdots \cup U_{h}$.

Definition 6.1 ( $H$-transversal family). A family $\mathcal{H}$ of pairwise vertex-disjoint copies of $H$ in $G\left[U_{1}, \ldots, U_{h}\right]$ is said to be a $H$-transversal family if, in addition, there exists a labelling $v_{1}, \ldots, v_{h}$ of the vertices of $H$ such that, for each copy $H \in \mathcal{H}$, the vertex $v_{i}$ is embedded into $U_{i}$ for each $i=1, \ldots, h$.

With $\mathcal{H}$ being a $H$-transversal family in $G$, we define the following bipartite auxiliary graph $\mathcal{T}_{G}(\mathcal{H}, V)$.

Definition 6.2 (Auxiliary graph $\mathcal{T}_{G}(\mathcal{H}, V)$ ). We define $\mathcal{T}_{G}(\mathcal{H}, V)$ to be the bipartite graph with partition classes $\mathcal{H}$ and $V$, where the edge between $H \in \mathcal{H}$ and $v \in V$ appears if and only if all vertices of $H$ are incident to $v$ in $G$.

We prove the following general lemma, which was already proved in the case when $H=K_{2}$ in [7, Lemma 8.1].

Lemma 6.3. Let $H$ be a graph and $h=v(H)$. For any $d, \delta, \varepsilon^{\prime}>0$ with $2 \delta \leq d$ there exist $\varepsilon, C>0$ such that the following holds. Let $V, U_{1}, \ldots, U_{h}$ be sets with $|V|=n$ and $\left|U_{i}\right|=m=\left(1 \pm \frac{1}{2}\right) n$ for $i=1, \ldots, h$ such that $\left(V, U_{i}\right)$ is $(\varepsilon, d)$-super-regular with respect to a graph $G$ for $i=1, \ldots, h$. Furthermore, suppose that $p \geq C n^{-1 / m_{1}(H)}$.

Then a.a.s. there exists an $H$-transversal family $\mathcal{H} \subseteq G\left(U_{1}, \ldots, U_{h}, p\right)$ of size $|\mathcal{H}| \geq$ $(1-\delta) m$ such that the pair $(\mathcal{H}, V)$ is $\left(\varepsilon^{\prime}, d^{h+1} 2^{-h-3}\right)$-super-regular with respect to the auxiliary graph $\mathcal{T}_{G}(\mathcal{H}, V)$.

The lemma shows that not only there is a large $H$-transversal family $\mathcal{H}$, but that we can additionally require that $(\mathcal{H}, V)$ is a super-regular pair in $\mathcal{T}_{G}(\mathcal{H}, V)$. The proof of Lemma 3.7 will use the special case of $H$ being the square of a path on $k$ vertices.

Before giving a proof of Lemma 6.3, we introduce an auxiliary $h$-partite $h$-uniform hypergraph $F=F_{G, V}\left(U_{1}, \ldots, U_{h}\right)$ to encode the potential tuples in $U_{1} \times \cdots \times U_{h}$ that we would like to use for building the copies of $H$ for the family $\mathcal{H}$.

Definition 6.4 (Auxiliary hypergraph $F$ ). We define $F=F_{G, V}\left(U_{1}, \ldots, U_{h}\right)$ to be the $h$-partite $h$-uniform hypergraph on $U_{1} \times \cdots \times U_{h}$ where a tuple $\left(u_{1}, \ldots, u_{h}\right) \in U_{1} \times \cdots \times U_{h}$ is an edge of $F$ if and only if the vertices $u_{1}, \ldots, u_{h}$ have at least $\frac{1}{2} d^{h} n$ common neighbours in the set $V$ in the graph $G$, i.e. $\left|\bigcap_{i=1}^{h} N_{G}\left(u_{i}, V\right)\right| \geq \frac{1}{2} d^{h} n$.

Similarly, given a set $X \subseteq V$, we call an edge $\left(u_{1}, \ldots, u_{h}\right) \in E(F) \operatorname{good}$ for $X$ if and only if there are at least $\frac{1}{2} d^{h}|X|$ vertices in $X$ that are incident to all of $u_{1}, \ldots, u_{h}$ in $G$. We denote by $F_{X}$ the spanning subgraph of $F$ with edges that are good for $X$.

However, we can only use those copies of $H$ which actually do appear in the random graph, and we encode that by defining the random spanning subgraph $\tilde{F}$ of $F$, as follows.

Definition 6.5 (Auxiliary hypergraph $\tilde{F}$ ). Let $F$ be the graph defined in Definition 6.4 . After revealing the edges of the random $h$-partite graph $G\left(U_{1}, \ldots, U_{h}, p\right)$, we denote by $\tilde{F}$ the (random) spanning subgraph of $F$ formed by those edges $\left(u_{1}, \ldots, u_{h}\right)$ of $F$ for which the vertices $u_{1}, \ldots, u_{h}$ give a copy of $H$ in the revealed random graph $G\left(U_{1}, \ldots, U_{h}, p\right)$. We will say that $\tilde{F}$ is supported by $G\left(U_{1}, \ldots, U_{h}, p\right)$. 
We remark that if $\left(u_{1}, \ldots, u_{h}\right) \in U_{1} \times \cdots \times U_{h}$ is an edge of $\tilde{F}$, then the vertices $u_{1}, \ldots, u_{h}$ give a copy of $H$ in $G\left(U_{1}, \ldots, U_{h}, p\right)$ and have at least $\frac{1}{2} d^{h} n$ common neighbours in the set $V$ in the graph $G$. We state some additional properties of $F$ below.

Lemma 6.6. Let $G$ be a graph on vertex set $V \cup U_{1} \cup \cdots \cup U_{h}$ with $|V|=n$ and $\left|U_{1}\right|=\cdots=$ $\left|U_{h}\right|=m=\left(1 \pm \frac{1}{2}\right) n$, and assume $\left(V, U_{i}\right)$ is a $(\varepsilon, d)$-super-regular pair with respect to $G$ for each $i=1, \ldots, h$. Let $F=F_{G, V}\left(U_{1}, \ldots, U_{h}\right)$ be the hypergraph defined in Definition 6.4. If $\varepsilon \leq \min \left\{\frac{1}{2} d^{h-1}, d\left(1-2^{-1 / h}\right)\right\}$, then the following holds:

(i) The minimum degree of $F$ is at least $(1-h \varepsilon) m^{h-1}$.

(ii) If $|X| \geq 2 \varepsilon n d^{1-h}$, all but at most $\varepsilon m$ vertices from each $U_{i}$ have degree at least $(1-h \varepsilon) m^{h-1}$ in $F_{X}$.

Moreover the subgraph $\tilde{F}$ keeps roughly the expected number of edges of $F$.

Lemma 6.7. For any graph $H$ on $h \geq 2$ vertices and any $\delta>0$ there exist $\varepsilon>0$ and $C>0$ such that the following holds for $p \geq C n^{-1 / m_{1}(H)}$. Let $F=F_{G, V}\left(U_{1}, \ldots, U_{h}\right)$ be the hypergraph defined in Definition 6.4. Then a.a.s. for any sets $U_{i}^{\prime} \subseteq U_{i}$ of size at least $\delta m$ for $i=1, \ldots, h$, we have that $\tilde{F}^{\prime}=\tilde{F}\left[U_{1}^{\prime}, \ldots, U_{h}^{\prime}\right]$ satisfies

$$
e\left(\tilde{F}^{\prime}\right)=(1 \pm \sqrt{\varepsilon}) \prod_{i=1}^{h}\left|U_{i}^{\prime}\right| p^{e(H)} .
$$

Moreover, if $|X| \geq 2 \varepsilon n d^{1-h}$, then with probability at least $1-e^{-n}$, for any choice of $U_{1}^{\prime}, \ldots, U_{h}^{\prime}$ as above and with $\tilde{F}_{X}^{\prime}=\tilde{F}_{X}\left[U_{1}^{\prime}, \ldots, U_{h}^{\prime}\right]$, we have

$$
e\left(\tilde{F}_{X}^{\prime}\right) \geq(1-\sqrt{\varepsilon}) \prod_{i=1}^{h}\left|U_{i}^{\prime}\right| p^{e(H)} .
$$

We remark that we will show a more general version of Lemma 6.7. Indeed our proof will only use that $F$ satisfies (i) and that $F_{X}$ satisfies (ii) for all $X$ with $|X| \geq 2 \varepsilon n d^{1-h}$. Thus (5) holds for all $h$-partite $h$-uniform hypergraph $F$ on partition classes $U_{1}, \ldots, U_{h}$ of size $m=\left(1 \pm \frac{1}{2}\right) n$ with minimum degree $(1-h \varepsilon) m^{h-1}$. Similarly (6) holds for all subgraphs $F_{X}$ of such $F$, such that all but $\varepsilon m$ vertices in each class have degree at least $(1-h \varepsilon) m^{h-1}$ in $F_{X}$.

The proof of Lemma 6.6 relies on a standard application of the regularity method, and that of Lemma 6.7 follows from an application of Chebyshev's and Janson's inequalities. Therefore we postpone them to Appendix A, and we now turn to main proof of this section.

Proof of Lemma 6.3. Given a graph $H$ on $h \geq 2$ vertices and $d, \delta, \varepsilon^{\prime}>0$ with $2 \delta \leq d$, suppose that

$$
\varepsilon \ll \eta \ll \gamma \ll d, \delta, \varepsilon^{\prime}
$$

are positive real numbers such that

$$
\gamma \leq \frac{1}{2} \varepsilon^{\prime}, \quad \eta \log \left(\frac{1}{\eta}\right) \leq \frac{1}{4} \gamma, \quad \varepsilon \leq \min \left\{\frac{1}{2} \eta d^{h-1}, d\left(1-2^{-1 / h}\right), \frac{1}{14^{2}} \gamma^{2}\right\},
$$

where additionally we require that $\varepsilon$ is small enough for Lemma 6.7 with input $H$ and $\delta$. Furthermore, let $C>0$ be large enough for Lemma 6.7 with the same input.

Given $n$, let $V, U_{1}, \ldots, U_{h}$ be sets of size $|V|=n$ and $\left|U_{i}\right|=m=\left(1 \pm \frac{1}{2}\right) n$ for $i=$ $1, \ldots, h$ such that $\left(V, U_{i}\right)$ is $(\varepsilon, d)$-super-regular with respect to a graph $G$ for $i=1, \ldots, h$. To find the family $\mathcal{H}$ we will now reveal edges of $G\left(U_{1}, \ldots, U_{h}, p\right)$ with probability $p \geq$ $C n^{-1 / m_{1}(H)}$ and consider the spanning subgraph $\tilde{F}$ of $F=F_{G, V}\left(U_{1}, \ldots, U_{h}\right)$ as defined in Definitions 6.4 and 6.5. Let $X \subseteq V$ be of size $\eta n$. With Lemma 6.7, we can assume that 
for all $U_{i}^{\prime} \subseteq U_{i}$ of size $\delta m$ for $i=1, \ldots, h$ we have that (5) and (6) hold, where the latter holds for all $X$ as above by a union bound.

Using a random greedy process we now choose a family of transversal copies $\mathcal{H}$ of $H$ of size $(1-\delta) m$ in $\tilde{F}$ as follows. Having chosen copies $H_{1}, \ldots, H_{t} \in \tilde{F}$ with $t<(1-\delta) m$, we pick $H_{t+1}$ uniformly at random from all edges of $\tilde{F}$ that do not share an endpoint with any of $H_{1}, \ldots, H_{t}$. This is possible since by $(5)$ there is always an edge in $\tilde{F}\left[U_{1}^{\prime}, \ldots, U_{h}^{\prime}\right]$ for any subsets $U_{i}^{\prime} \subseteq U$ of size at least $\delta m$ for $i=1, \ldots, h$, and thus a transversal copy of $H$ in $G\left(U_{1}, \ldots, U_{h}, p\right)$. For $i=1, \ldots, t$, we denote the $i$-th chosen copy of $H$ for $\mathcal{H}$ by $H_{i}$, and by $\mathcal{H}_{i}$ the history $H_{1}, \ldots, H_{i}$. It remains to show that $(\mathcal{H}, V)$ is $\left(\varepsilon^{\prime}, 2^{-h-3} d^{h+1}\right)$-super-regular with respect to the auxiliary graph $\mathcal{T}=\mathcal{T}_{G}(\mathcal{H}, V)$.

Observe that any $H \in \mathcal{H}$ has $\left|N_{\mathcal{T}}(H)\right| \geq \frac{1}{2} d^{h} n \geq d^{h+1} 2^{-h-3}|V|$ by construction. Moreover for any $v \in V$ we have $\left|N_{H}(v)\right| \geq 2^{-h-3} d^{h+1} m$; this can be shown as follows. Consider the first $\frac{1}{2} d m$ chosen copies, then for $i=1, \ldots, \frac{1}{2} d m$, by (5), there are at most $(1+$ $\sqrt{\varepsilon}) \prod_{i=1}^{h}\left|U_{i}\right| p^{e(H)}$ available copies to chose $H_{i}$ from. On the other hand, as long as $i<\frac{1}{2} d m$, the vertex $v$ has at least $\frac{1}{2} d m \geq \delta m$ neighbours $U_{i}^{\prime} \subseteq U_{i}$ for $i=1, \ldots, h$ that are not covered by the edges in $\mathcal{H}_{i-1}$. Therefore, by (5) there are at least $(1-\sqrt{\varepsilon}) \prod_{i=1}^{h}\left|U_{i}^{\prime}\right| p^{e(H)}$ choices for $H_{i}$ such that $H_{i} \in N_{\mathcal{T}}(v)$.

Hence, for $i=1, \ldots, \frac{1}{2} d n$, we get

$$
\mathbb{P}\left[H_{i} \in N_{\mathcal{T}}(v) \mid \mathcal{H}_{i-1}\right] \geq \frac{(1-\sqrt{\varepsilon}) \prod_{i=1}^{h}\left|U_{i}^{\prime}\right| p^{e(H)}}{(1+\sqrt{\varepsilon}) \prod_{i=1}^{h}\left|U_{i}\right| p^{e(H)}} \geq \frac{(1-\sqrt{\varepsilon})\left(\frac{1}{2} d\right)^{h}}{(1+\sqrt{\varepsilon})} \geq 2^{-h-1} d^{h} .
$$

As this holds independently of the history of the process, this process dominates a binomial distribution with parameters $\frac{1}{2} d m$ and $2^{-h-1} d^{h}$. Therefore, even though the events are not mutually independent, we can use Chernoff's inequality (Lemma 2.1) to infer that $\left|N_{\mathcal{T}}(v)\right| \geq 2^{-h-3} d^{h+1} m$ with probability at least $1-n^{-2}$. Then, by applying the union bound over all $v \in V$, we obtain that a.a.s. $\left|N_{\mathcal{T}}(v)\right| \geq 2^{-h-3} d^{h+1} m \geq 2^{-h-3} d^{h+1}|\mathcal{H}|$ for all $v \in V$.

Next let $X \subseteq V$ be any subset with $|X|=\eta n$, and let $t=(1-\delta) m$. For $i=0,1, \ldots, t-1$, we obtain from (5) that there are at most $(1+\sqrt{\varepsilon}) \prod_{i=1}^{h}\left|U_{i}^{\prime}\right| p^{e(H)}$ edges in $\tilde{F} \backslash \mathcal{H}_{i}$ available for choosing $H_{i+1}$, of which, by $(6)$, at least $(1-\sqrt{\varepsilon}) \prod_{i=1}^{h}\left|U_{i}^{\prime}\right| p^{e(H)}$ are in $\tilde{F}_{X}$. Then

$$
\mathbb{P}\left[H_{i} \text { good for } X \mid \mathcal{H}_{i-1}\right] \geq \frac{(1-\sqrt{\varepsilon}) \prod_{i=1}^{h}\left|U_{i}^{\prime}\right| p^{e(H)}}{(1+\sqrt{\varepsilon}) \prod_{i=1}^{h}\left|U_{i}^{\prime}\right| p^{e(H)}} \geq(1-2 \sqrt{\varepsilon}) .
$$

Again, as the lower bound on the probability holds independently of the history of the process, this process dominates a binomial distribution with parameters $(1-\delta) m$ and $(1-2 \sqrt{\varepsilon})$. We let $B_{X} \subseteq \mathcal{H}$ be the copies in $\mathcal{H}$ that are not good for $X$ and deduce

$$
\mathbb{E}\left[\left|B_{X}\right|\right] \leq(1-\delta) m 2 \sqrt{\varepsilon} \leq 2 \sqrt{\varepsilon} m .
$$

Then we get from Chernoff's inequality (Lemma 2.1) that, since $\gamma m \geq 14 \sqrt{\varepsilon} m \geq 7 \mathbb{E}\left[\left|B_{X}\right|\right]$, we have

$$
\mathbb{P}\left[\left|B_{X}\right|>\gamma m\right] \leq \exp (-\gamma m) .
$$

There are at most $\left(\begin{array}{c}n \\ \eta n\end{array}\right) \leq\left(\frac{e}{\eta}\right)^{\eta n} \leq \exp \left(\eta \log \left(\frac{1}{\eta}\right) n\right) \leq \exp \left(\frac{1}{2} \gamma m\right)$ choices for $X$ and, thus, with the union bound over all these choices, we obtain that a.a.s. there are at most $\gamma m$ bad copies in $\mathcal{H}$ for any $X \subseteq V$ with $|X|=\eta n$.

Then for any set $X^{\prime} \subseteq V$ and $\mathcal{H}^{\prime} \subseteq \mathcal{H}$ with $\left|X^{\prime}\right| \geq \varepsilon^{\prime} n$ and $\left|\mathcal{H}^{\prime}\right| \geq \varepsilon^{\prime}|\mathcal{H}|$ we find

$$
e_{\mathcal{T}}\left(\mathcal{H}^{\prime}, X^{\prime}\right) \geq\left(\left|\mathcal{H}^{\prime}\right|-\gamma m\right) \frac{d^{h} \eta n}{2} \frac{\left|X^{\prime}\right|}{2 \eta n} \geq \frac{d^{h}}{8}\left|\mathcal{H}^{\prime}\right|\left|X^{\prime}\right|
$$


by partitioning $X^{\prime}$ into pairwise disjoint sets of size $\eta n$. This implies that $(\mathcal{H}, V)$ is $\left(\varepsilon^{\prime}, d^{h+1} 2^{-h-3}\right)$-super-regular with respect to $\mathcal{T}_{G}(\mathcal{H}, V)$.

\section{Proof of AUXILIARY LEMmaS}

In this section we prove Lemma 3.7 and then derive Lemma 3.8. Throughout the section we denote the square of a path on $k$ vertices by $H^{(k)}$, and we we list their vertices as $u_{1}, \ldots, u_{k}$, meaning that the edges of the square are $u_{i} u_{j}$ for each $1 \leq|i-j| \leq 2$. We start with a short overview of our argument for the proof of Lemma 3.7, where we want to find the square of a Hamilton path, covering $V, U_{1}, \ldots, U_{k}$ and with end-tuples $\left(x, x^{\prime}\right)$ and $\left(y, y^{\prime}\right)$. Our proof will follow four steps, and the decomposition of this square of a Hamilton path in random and deterministic edges is outlined in Figure 6.

Segment 1

Segment 3

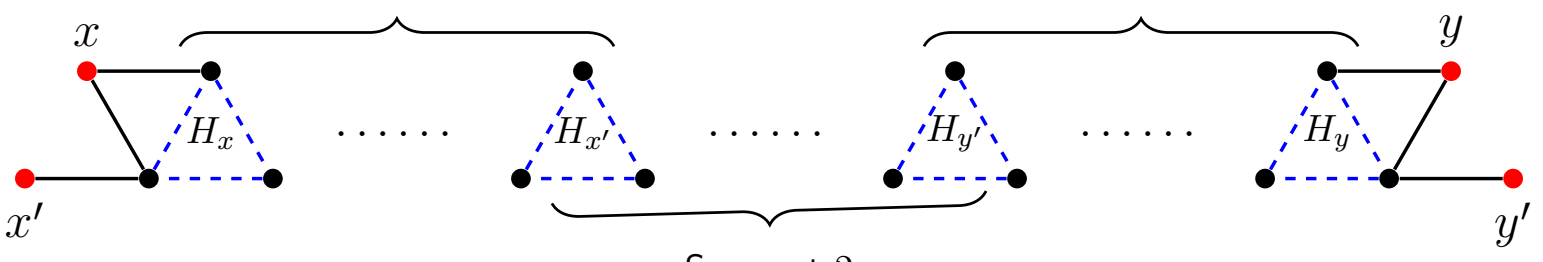

Segment 2

Figure 6. The square of a Hamilton path with endtuples $\left(x, x^{\prime}\right)$ and $\left(y, y^{\prime}\right)$ in Lemma 3.7 and its decomposition into edges from $V$ (black) and from $G(n, p)$ (dashed blue). Each dotted $H_{x}, H_{x^{\prime}}, H_{y^{\prime}}, H_{y}$ stands for a copy of $P_{k}^{2}$ with edges all from $G(n, p)$. Segment 1 and 2 (resp. segment 3 ) are realised through several copies of the structure in Figure 8 (resp. Figure 7).

To ensure that $\left(x, x^{\prime}\right)$ and $\left(y, y^{\prime}\right)$ are the end-tuples of the square of the path, we will first find copies $H_{x}$ and $H_{y}$ of $H^{(k)}$ that are connected to the tuples $\left(x, x^{\prime}\right)$ and $\left(y, y^{\prime}\right)$ (c.f. Figure 6). Moreover, with Lemma 6.3, we will find a large family $\mathcal{H}$ of transversal copies of $H^{(k)}$ in $U_{1}, \ldots, U_{k}$ (c.f. Definition 6.1) such that $(\mathcal{H}, V)$ is super-regular with respect to the auxiliary graph $\mathcal{T}_{G}(\mathcal{H}, V)$ (c.f. Definition 6.2). In particular, this will guarantee that most pairs $\left(H, H^{\prime}\right) \in \mathcal{H}^{2}$ have many common neighbours in $V$ in the graph $G$.

The next step is to find random edges between the copies in $\mathcal{H}$. For that we will consider a directed auxiliary graph $\bar{F}$ with vertex set $\mathcal{H}$ where, given $H, H^{\prime} \in \mathcal{H}$, with $H$ on $u_{1}, \ldots, u_{k}$ and $H^{\prime}$ on $u_{1}^{\prime}, \ldots, u_{k}^{\prime}$, the pair $\left(H, H^{\prime}\right)$ is an edge of $\bar{F}$ if and only if $u_{k} u_{1}^{\prime}$ is a random edge and $V(H) \cup V\left(H^{\prime}\right)$ have many common neighbours in $V$ in the graph $G$. This will allow us to connect $H$ to $H^{\prime}$ with a random edge, while also giving many choices for vertices from $V$ to turn this into the square of a path on $2 k+1$ vertices (c.f. Figure 7). We will use a random greedy procedure to find a long directed path $D$ in $\bar{F}$ that covers most of $\mathcal{H}$, which is possible by the properties of $\mathcal{H}$ and our choice of $p \geq C n^{-1}$. Additionally, we can guarantee that we will later be able to extend this into the square of a path using any subset of vertices $V^{\prime} \subseteq V$ of the right size. We denote the first and last copy of the path $D$ by $H_{x}^{\prime}$ and $H_{y}^{\prime}$, respectively.

In the next step, we take care of the set $Z$ of those vertices in $U_{1} \cup \cdots \cup U_{k}$ that are not covered by any copy of $H^{(k)}$ from $\mathcal{H}$. We will absorb each vertex $z \in Z$ into the square of a short path, using four vertices from $V$, two copies of $H^{(k)}$ in $\mathcal{H} \backslash V(D)$, and random edges within $V$ (c.f. Figure 8). In fact, we will be able to do that simultaneously for each vertex in $Z$, while also covering all copies of $H^{(k)}$ in $\mathcal{H} \backslash V(D)$, and using only two squares of paths, one from $H_{x}$ to $H_{x}^{\prime}$ and one from $H_{y}^{\prime}$ to $H_{y}$. 


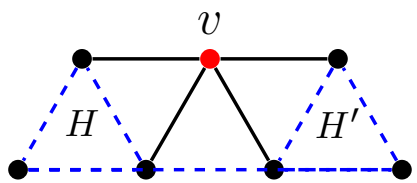

Figure 7. Connecting two copies $H$ and $H^{\prime}$ of $P_{k}^{2}$, using one vertex $v \in V$ (red), edges from $G(n, p)$ (dashed blue), and edges from $G$ (black).

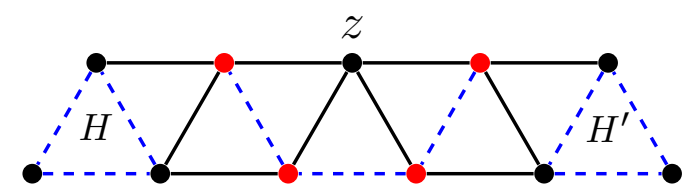

Figure 8. Absorbing a vertex $z \in$ $Z \subseteq U_{1} \cup \cdots \cup U_{k}$, using two copies $H$ and $H^{\prime}$ of $P_{k}^{2}$, four vertices from $V$ (red), edges from $G(n, p)$ (dashed blue), and edges from $G$ (black).

In the final step, we will find a perfect matching between the edges $\left(H, H^{\prime}\right)$ of $D$ and the remaining vertices of $V$, where we will make sure that the sizes of the two sets we want to match are the same. A vertex $v \in V$ can be matched to $\left(H, H^{\prime}\right)$ if and only if $u_{k}, u_{k-1}, u_{1}^{\prime}, u_{2}^{\prime}$ are neighbours of $v$ in $G$ (with the labelling of the vertices of $H, H^{\prime}$ as above). This matching will close the gap between the two copies $H$ and $H^{\prime}$ for each edge $\left(H, H^{\prime}\right)$ of $D$ with a vertex $v$ from $V$. This will give the square of a path from $H_{x}^{\prime}$ to $H_{y}^{\prime}$ and, together with the other pieces from $H_{x}$ to $H_{x}^{\prime}$ and from $H_{y}$ to $H_{y}^{\prime}$, we will get the square of a Hamilton path with the correct end-tuples. Ultimately, the shape of this square of the path is as illustrated in Figure 6, where the segments between $H_{x}$ and $H_{x^{\prime}}$ and between $H_{y^{\prime}}$ and $H_{y}$ (resp. between $H_{x^{\prime}}$ and $H_{y^{\prime}}$ ) are obtained by repeatedly inserting Figure 8 (resp. Figure 7) several times. We will now turn to the details of the argument.

Proof of Lemma 3.7. Given an integer $k \geq 2$, let $H^{(k)}$ be the square of a path on $k$ vertices and observe that $m_{1}\left(H^{(k)}\right)=\frac{2 k-3}{k-1}$. Given $0<\delta^{\prime} \leq d \leq 1$, let $\delta_{1}, \delta_{0}, \varepsilon^{\prime}>0$ with $2 \delta_{1}<\delta_{0}<\min \left\{\delta^{\prime}, d^{3 k+3} 2^{-3 k-20}\right\}$ and $\varepsilon^{\prime}<\delta_{0}^{8}$. Let $C_{2.2}$ be given by Lemma 2.2 for input $2 \delta_{1}$ and $F$, where $F$ is the path on four vertices. Then let $\varepsilon_{6.3}$ and $C_{6.3}$ be given by Lemma 6.3 for input $H^{(k)}, d, \varepsilon^{\prime}$, and $\varepsilon^{\prime}$, where $\varepsilon^{\prime}$ plays the role of $\delta$ in the statement of Lemma 6.3. Let $\varepsilon_{6.7}$ and $C_{6.7}$ be given by Lemma 6.7 with input $H^{(k)}$ and $\delta_{0}$. Finally let $\varepsilon<\min \left\{\varepsilon_{6.3}, \varepsilon_{6.7}, \varepsilon^{\prime} / 4\right\}$ and $C=\max \left\{C_{2.2}, 2 C_{6.3}, 2 C_{6.7}, 48 \varepsilon^{\prime-1} \delta^{-1}\right\}$.

Let $G$ be a graph on $V \cup U_{1} \cup \cdots \cup U_{k}$, where $V, U_{1}, \ldots, U_{k}$ are pairwise disjoint sets of size $|V|=n+4$ and $\left(1-\delta_{0}\right) n \leq\left|U_{i}\right|=m \leq\left(1-\delta_{1}\right) n$ for $i=1, \ldots, n$ such that $n-m \equiv 0$ $(\bmod 6 k-2)$. Suppose that $\left(V, U_{i}\right)$ is a $(\varepsilon, d)$-super-regular pair for $i=1, \ldots, k$. Further let $\left(x, x^{\prime}\right)$ and $\left(y, y^{\prime}\right)$ two tuples from $V$ such that both tuples have $d^{2} m / 2$ common neighbours in $U_{i}$ for $i=1, \ldots, k$ in the graph $G$. Let $\delta$ be such that $m=(1-\delta) n+2$ and observe that $\delta_{1} \leq \delta \leq \delta_{0}+2 / n$. We reveal random edges in three rounds $G_{0} \sim G\left(U_{1}, \ldots, U_{k}, \frac{1}{2} p\right)$, $G_{1} \sim G\left(U_{1}, \ldots, U_{k}, \frac{1}{2} p\right)$, and $G_{2} \sim G(V, p)$, where $p \geq C n^{-(k-1) /(2 k-3)}$. Moreover we assume that a.a.s. the events of Lemma 2.2 and 6.7 hold in $G_{2}$ and $G_{0}$, respectively.

Finding transversal copies of $H^{(k)}$. We start by ensuring that $\left(x, x^{\prime}\right)$ and $\left(y, y^{\prime}\right)$ can be the end-tuples of the square of a path. Fix $i=1, \ldots, k$. Recall that each of the tuples $\left(x, x^{\prime}\right)$ and $\left(y, y^{\prime}\right)$ has $d^{2} m / 2$ common neighbours in $U_{i}$. Thus we can pick disjoint sets $U_{i, x}, U_{i, y} \subseteq U_{i}$ of size $d^{2} m / 4$ such that $U_{i, x}$ and $U_{i, y}$ are in the common neighbourhoods of $\left(x, x^{\prime}\right)$ and $\left(y, y^{\prime}\right)$ in $U_{i}$, respectively. Let $F$ and $\tilde{F}$ be the hypergraphs defined in Definition 6.4 , and with $H^{(k)}$ and $\tilde{F}$ supported by $G_{0}$. Then with Lemma 6.6 (ii) and Lemma 6.7 we a.a.s. find and edge in $\tilde{F}\left[U_{1, x}, \ldots, U_{k, x}\right]$ and an edge in $\tilde{F}\left[U_{1, y}, \ldots, U_{k, y}\right]$. Given the definition of $\tilde{F}$, these edges corresponds to copies of $H^{(k)}$ in $G_{0}$ and we denote them by $H_{x}=\left(x_{1}, \ldots, x_{k}\right)$ and $H_{y}=\left(y_{1}, \ldots, y_{k}\right)$.

For $i=1, \ldots, k$, let $U_{i}^{\prime}=U_{i} \backslash\left\{x_{i}, y_{i}\right\}$ and observe that $\left|U_{i}^{\prime}\right|=(1-\delta) n$ and $\left(U_{i}^{\prime}, V\right)$ is $\left(2 \varepsilon, \frac{1}{2} d\right)$-super-regular. Then we apply Lemma 6.3 with $G_{1}\left[U_{1}^{\prime}, \ldots, U_{k}^{\prime}\right]$ to a.a.s. obtain 
a family $\mathcal{H}$ of transversal copies of $H^{(k)}$, of size $|\mathcal{H}| \geq\left(1-\varepsilon^{\prime}\right)(m-2) \geq\left(1-\frac{3 k}{3 k-1} \delta\right) n$ and such that the pair $(\mathcal{H}, V)$ is $\left(\varepsilon^{\prime}, d^{k+1} 2^{-k-3}\right)$-super-regular with respect to $\mathcal{T}_{G}(\mathcal{H}, V)$, where $\mathcal{T}_{G}(\mathcal{H}, V)$ is the graph defined in Definition 6.2. By removing some copies of $H^{(k)}$, we can assume that $|\mathcal{H}|=m_{0}=\left(1-\frac{3 k}{3 k-1} \delta\right) n$ and still have that the pair $(\mathcal{H}, V)$ is $\left(\varepsilon^{\prime}, d^{k+1} 2^{-k-4}\right)$ super-regular with respect to $\mathcal{T}_{G}(\mathcal{H}, V)$.

Building the directed path $D$. Ultimately we want to find a directed path $D$, that has some of the copies of $H^{(k)}$ in $\mathcal{H}$ as vertices. As we later would like to connect two copies of $H^{(k)}$ by one random edge and a vertex from $V$ to get the square of a path on $2 k+1$ vertices (c.f. Figure 7), we want them to appear consecutively in $D$ only if all their vertices have enough common neighbours in $V$ in the graph $G$. We encode this condition in the auxiliary graph $F^{*}$ with vertex set $\mathcal{H}$, and where, given $H, H^{\prime} \in \mathcal{H}$, the edge $H H^{\prime}$ is in $F^{*}$ if and only if the vertices in $V(H) \cup V\left(H^{\prime}\right)$ have at least $d^{2 k+2} 2^{-2 k-7} n$ common neighbours in $V$ in the graph $G$.

Claim 7.1. The minimum degree of $F^{*}$ is at least $\left(1-2 \varepsilon^{\prime}\right) m_{0}$.

Proof of Claim \%.1. Any copy of $H \in \mathcal{H}$ has degree at least $d^{k+1} 2^{-k-3} n$ into $V$ in the graph $\mathcal{T}_{G}(\mathcal{H}, V)$. Then, by super-regularity in $\mathcal{T}_{G}(\mathcal{H}, V)$, all but $2 \varepsilon^{\prime} m_{0}$ copies of $H^{\prime} \in \mathcal{H}$ have at least $d^{2 k+2} 2^{-2 k-7} n$ common neighbours with $H$. This implies that $H$ has degree $\left(1-2 \varepsilon^{\prime}\right) m_{0}$ in $F^{*}$.

For a set $X \subseteq V$, we call an edge $H H^{\prime} \in E\left(F^{*}\right) \operatorname{good}$ for $X$ if there is at least one vertex $v \in X$ that is incident to $H$ and $H^{\prime}$ in $\mathcal{T}_{G}(\mathcal{H}, V)$. We denote the subgraph of $F^{*}$ with edges that are good for $X$ by $F_{X}^{*}$.

Claim 7.2. If $|X| \geq d^{-k-1} 2^{k+4} \varepsilon^{\prime} n$, then all but at most $\varepsilon^{\prime} n$ vertices of $\mathcal{H}$ have degree at least $\left(1-4 \varepsilon^{\prime}\right) m_{0}$ in $F_{X}^{*}$.

Proof of Claim 7.2. All but at most $\varepsilon^{\prime} n$ copies $H \in \mathcal{H}$ have degree at least $d^{k+1} 2^{-k-4} n|X| \geq$ $\varepsilon^{\prime} n$ into $X$ in the graph $\mathcal{T}_{G}(\mathcal{H}, V)$. Fixing any $H \in \mathcal{H}$ with this property, all but at most $\varepsilon^{\prime} m_{0}$ copies $H^{\prime} \in \mathcal{H} \backslash\{H\}$ have at least one common neighbour with $H$ in $X$. From Claim 7.1 we know that $\delta\left(F^{*}\right) \geq\left(1-2 \varepsilon^{\prime}\right) m_{0}$ and, therefore, all but at most $\varepsilon^{\prime} n$ vertices from $\mathcal{H}$ have degree at least $\left(1-4 \varepsilon^{\prime}\right) m_{0}$ in $F_{X}^{*}$.

We now define an auxiliary directed graph $\bar{F}$ on vertex set $\mathcal{H}$ as follows. Given any $H$ and $H^{\prime} \in \mathcal{H}$ with $H=u_{1}, \ldots, u_{k}$ and $H^{\prime}=u_{1}^{\prime}, \ldots, u_{k}^{\prime}$, the pair $\left(H, H^{\prime}\right)$ is a directed edge of $\bar{F}$ if and only if $H H^{\prime}$ is an edge of $F^{*}$ (which means that $H$ and $H^{\prime}$ have many common neighbours in $V$ in $G$ ) and $u_{k} u_{1}^{\prime}$ is an edge of $G_{1}$. Note that for each edge $H H^{\prime}$ of $F^{*}$ we get the directed edge $\left(H, H^{\prime}\right)$ with probability $p / 2$ independently of all the other edges (in particular, independently of the edge $\left.\left(H^{\prime}, H\right)\right)$. Therefore, we can also reveal each edge $\left(H, H^{\prime}\right)$ in $F^{*}$ with probability $p / 2$ independently of all the others and every edge of $G_{1}$ will be revealed with probability at most $p / 2$ independently of all the others. We denote the resulting directed graph by $\bar{F}$ and we want to find a long directed path $D$ in $\bar{F}$.

For this we will use a random greedy process that explores the graph using a depth-first search algorithm. We do not reveal all the edges of $F^{*}$ at the beginning, but, at each step of the algorithm, we only reveal those edges that are relevant for that step, and add those which are successful to $\bar{F}$. In each step the algorithm maintains a directed path $H_{1}, \ldots, H_{s}$ in $\bar{F}$ and the set $B$ of the vertices in $\mathcal{H} \backslash\left\{H_{1}, \ldots, H_{s}\right\}$, whose neighbours have already been all revealed (we call them "dead-ends"). Additionally, we keep track of the vertices which have already been visited at least twice (due to back tracking of the algorithm) and denote their set by $A$. We initialise $s=0, A=\varnothing$, and $B=\varnothing$. 
The algorithms proceeds as follows. If $s=0$, then we choose an arbitrary vertex $H_{1} \in \mathcal{H} \backslash B$ and increase $s$ by one. If $s>0$, we let $\mathcal{H}^{\prime}=\mathcal{H} \backslash\left(\left\{H_{1}, \ldots, H_{s}\right\} \cup B\right)$ be the vertices that have not been used and that are not "dead-ends". If $H_{s} \notin A$, then from $F^{*}$ we reveal all directed edges from $H_{s}$ to $\mathcal{H}^{\prime}$ with probability $p / 2$. If possible, we pick one uniformly at random among all those that are successful, denote it by $H_{s+1}$, and increase $s$ by one. If none of them is successful, we add $H_{s}$ to $B, H_{s-1}$ to $A$, and decrease $s$ by one. If $H_{s} \in A$, then the directed edges from $H_{s}$ to $\mathcal{H}^{\prime}$ in $F^{*}$ have already been revealed earlier. If there is an edge from $H_{s}$ to some $H^{\prime} \in \mathcal{H}^{\prime}$ in $\bar{F}$, we let $H_{s+1}=H^{\prime}$ and increase $s$ by one. Otherwise, we add $H_{s}$ to $B, H_{s-1}$ to $A$, and decrease $s$ by one. The algorithms stops if $s=\left(1-\frac{4 k}{3 k-1} \delta\right) n$ or when $|B| \geq \varepsilon^{\prime} n$, whichever happens first. We claim that the algorithm terminates and the latter does not happen.

Claim 7.3. The graph $\bar{F}$ a.a.s. contains a directed path $D$ on $t=\left(1-\frac{4 k}{3 k-1} \delta\right) n$ vertices.

Proof of Claim 7.3. First, we observe that the algorithm terminates. Indeed, if $|B|<\varepsilon^{\prime} n$ then $\left|\mathcal{H}^{\prime}\right| \geq|\mathcal{H}|-t-|B|>2 \varepsilon^{\prime} m_{0}$ and with Claim 7.1 there is at least one edge of $F^{*}$ from $H_{s}$ to $\mathcal{H}^{\prime}$. Secondly, we claim that a.a.s. $|B|<\varepsilon^{\prime} n$. Assume that at some point we have $|B|=\varepsilon^{\prime} n$ and $s \leq\left(1-\frac{4 k}{3 k-1} \delta\right) n$. Since at least $|\mathcal{H}|-s-|B| \geq \delta n / 4$ vertices of $\mathcal{H}$ are not covered by the path or a vertex from $B$, we can pick a set $\mathcal{H}^{\prime}$ of exactly $\delta n / 4$ of them. This implies that all edges from $B$ to $\mathcal{H}^{\prime}$ that are in $F^{*}$ have been revealed but none is present in $\bar{F}$. However, with Claim 7.1, the expected number of edges in $\bar{F}$ from $B$ to $\mathcal{H}^{\prime}$ with $|B|=\varepsilon^{\prime} n$ and $\left|\mathcal{H}^{\prime}\right|=\frac{1}{4} \delta n$ is $\frac{1}{8} p \varepsilon^{\prime} \delta n^{2}$ and by Chernoff's inequality (Lemma 2.1) the probability that none of the edges in $F^{*}$ from $B$ to $\mathcal{H}^{\prime}$ appears in $\bar{F}$ is at most $2 \exp \left(-\frac{1}{24} \varepsilon^{\prime} p \delta n^{2}\right) \leq 2 \exp (-2 n)$. A union bound over the at most $2^{2 n}$ choices for $B$ and $\mathcal{H}^{\prime}$ implies that the probability that there exist $B$ and $\mathcal{H}^{\prime}$ as above is $o(1)$. Therefore the process stops when $s=t$ and we obtain a directed path $D$ on $t$ vertices in $\bar{F}$.

Preparing the final matching. The previous claim already guarantees a long directed path, but we need some additional properties that enable us to finish the proof later. An edge of $D$ corresponds to an edge $H H^{\prime}$ of $F^{*}$ and, for each of them, there are many choices for a vertex $v \in V$ that turns this into the square of a path on $2 k+1$ vertices. We will need to do this simultaneously for all edges of $D$ in the last step of the proof. However, before the last step, we have to cover the the leftover of $U_{1} \cup \cdots \cup U_{k}$ and $\mathcal{H}$, which will be possible by using some vertices of $V$. This will leave a subset $V^{\prime} \subseteq V$ of size $t$ to match to the edges of $D$ in the last step. We now show that this is possible for any subset $V^{\prime} \subseteq V$ of size $t$. We encode this task as follows. Given a vertex $v \in V$, we define $\mathcal{F}_{v}$ to be the set of all pairs $\left(H, H^{\prime}\right) \in \mathcal{H}^{2}$ such that both $H$ and $H^{\prime}$ are adjacent to $v$ in $\mathcal{T}_{G}(\mathcal{H}, V)$; note that this means that $v$ is adjacent to all vertices in $V(H) \cup V\left(H^{\prime}\right)$ in the graph $G$. Then we define a bipartite auxiliary graph $\mathcal{F}_{D}$ with partition $E(D)$ and $V$, where for $e \in E(D)$ and $v \in V$, the pair $e v$ is an edge of $\mathcal{F}_{D}$ if and only if $e \in \mathcal{F}_{v}$.

Claim 7.4. A.a.s. for any $V^{\prime} \subseteq V$ of size $t$ the graph $\mathcal{F}_{D}\left[V^{\prime}, E(D)\right]$ contains a perfect matching.

Proof of Claim \%.4. It suffices to show that for each $X \subseteq V$ of size at most $t$ we have $\left|\bigcup_{v \in X} N_{\mathcal{F}_{D}}(v)\right| \geq|X|$ and then the result follows by Hall's condition. We will use the notation from the algorithm introduced above.

Let $X \subseteq V$ of size at most $t$ be given. First suppose that $|X|>\left(1-d^{k+2} 2^{-2 k-8}\right) n$ and let $e=\left(H, H^{\prime}\right)$ be any edge of $D$. Since $H H^{\prime}$ is in particular an edge of $F^{*}$, the vertices $V(H) \cup V\left(H^{\prime}\right)$ have at least $d^{k+2} 2^{-2 k-7} n$ neighbours in $V$. As $\left|V \backslash V^{\prime}\right| \leq 2 \delta n \leq d^{k+2} 2^{-2 k-8} n$, $e$ has a neighbour in $X$ with respect to $\mathcal{F}_{D}$. Since this is true for any edge $e$ of $D$, we conclude that $\left|\bigcup_{v \in X} N_{\mathcal{F}_{D}}(v)\right|=t \geq|X|$. 
Secondly suppose that $|X|<\delta n$. Here it suffices to show that for any $v \in V$ we have $\left|N_{\mathcal{F}_{D}}(v)\right| \geq \delta n$. Fix any $v \in V$ and let $\ell=d^{k+1} 2^{-k-6} n$. As the pair $(\mathcal{H}, V)$ is $\left(\varepsilon^{\prime}, d^{k+1} 2^{-k-4}\right)$ super-regular with respect to $\mathcal{T}_{G}(\mathcal{H}, V)$, we have that $v$ has degree at least $d^{k+1} 2^{-k-4} m_{0}$ into $\mathcal{H}$ with respect to $\mathcal{T}_{G}(\mathcal{H}, V)$. Consider any point during the first $\ell$ steps of the algorithm and let $H_{1}, \ldots, H_{s}$ be the current path. We assume that $H_{s}$ is (not yet) in $A$ and denote by $\mathcal{M}$ the history of the algorithm until this point. Now we will look into the next two steps of the algorithm and estimate the probability such that two vertices $H_{s+1}$ and $H_{s+2}$ are chosen and such that the edge $\left(H_{s+1}, H_{s+2}\right)$ is in $\mathcal{F}_{v}$.

The first part is equivalent to $H_{s} \notin B$ and $H_{s+1} \notin B$ and with $\mathbb{P}\left[H_{s} \in B\right] \leq\left(1-\frac{p}{2}\right)^{\delta n} \leq$ $\exp \left(-\frac{p}{2} \delta n\right) \leq \varepsilon^{\prime}$ we get that $\mathbb{P}\left[H_{s}, H_{s+1} \notin B\right] \geq\left(1-\varepsilon^{\prime}\right)^{2}$. Next, we want to bound the number of valid choices for $H_{s+1}$ in $\mathcal{H}^{\prime}$ that are neighbours of $v$ in $\mathcal{T}_{G}(\mathcal{H}, V)$. From the neighbours of $v$ in $\mathcal{T}_{G}(\mathcal{H}, V)$, we have to exclude those $H^{\prime}$ such that $H_{s} H^{\prime}$ is not an edge of $F^{*}$, and those $H^{\prime}$ that are "dead-ends": in the first case their number is at most $2 \varepsilon^{\prime} m_{0}$ by Claim 7.1, in the second case their number is at most $|B|<\varepsilon^{\prime} n$. Therefore there are at least $d^{k+1} 2^{-k-4} m_{0}-2 \varepsilon^{\prime} m_{0}-\varepsilon^{\prime} n-\ell \geq d^{k+1} 2^{-k-5} m_{0}$ valid choices for $H_{s+1}$ in $\mathcal{H}^{\prime}$ that are neighbours of $v$ in $\mathcal{T}_{G}(\mathcal{H}, V)$. Repeating the same argument in the next step of the algorithm, there are at least $d^{k+1} 2^{-k-5} m_{0}$ valid choices for $H_{s+2}$ in $\mathcal{H}^{\prime}$ that are neighbours of $v$ in $\mathcal{T}_{G}(\mathcal{H}, V)$. In particular for such choices of $H_{s+1}$ and $H_{s+2}$, the edge $\left(H_{s+1}, H_{s+2}\right)$ is in $\mathcal{F}_{v}$.

We have that $H_{s}$ (resp. $H_{s+1}$ ) is not in $A$ and we condition on the event that it is also not in $B$. Since we revealed the edges of $F^{*}$ in each step separately the vertex $H_{s+1}$ (resp. $H_{s+2}$ ) is then chosen uniformly at random from the at most $|\mathcal{H}|=m_{0}$ available possibilities. Therefore,

$$
\mathbb{P}\left[\left(H_{s+1}, H_{s+2}\right) \in \mathcal{F}_{v} \mid \mathcal{M} \wedge H_{s}, H_{s+1} \notin B\right] \geq \frac{\left(d^{k+1} 2^{-k-5} m_{0}\right)^{2}}{m_{0}^{2}} \geq d^{2 k+2} 2^{-2 k-10}
$$

and together with the bound on $\mathbb{P}\left[H_{s}, H_{s+1} \notin B\right]$ we get

$$
\mathbb{P}\left[\left(H_{s+1}, H_{s+2}\right) \in \mathcal{F}_{v} \mid \mathcal{M}\right] \geq\left(1-\varepsilon^{\prime}\right)^{2} d^{2 k+2} 2^{-2 k-10} \geq d^{2 k+2} 2^{-2 k-11} .
$$

Crucially, this lower bound holds independently of the history $\mathcal{M}$. As among the first $\ell$ steps we can have at most $\varepsilon^{\prime} n$ many steps in which $H_{s} \in A$, this process dominates a binomial distribution with parameters $\ell-\varepsilon^{\prime} n$ and $d^{2 k+2} 2^{-2 k-11} n$. Therefore, even though the events are not mutually independent, we can use Chernoff's inequality (Lemma 2.1) to infer that with probability at least $1-n^{-2}$ at least $d^{3 k+3} 2^{-3 k-18} n$ of these edges are in $\mathcal{F}_{v}$. Some of these edges might not appear in the final path $D$, beacuse of the "dead-ends" and the back tracking of the algorithm, but their number is at most $\varepsilon^{\prime} n$. Thus we get that $\left|N_{\mathcal{F}_{D}}(v)\right| \geq d^{3 k+3} 2^{-3 k-18} n-\varepsilon^{\prime} n \geq \delta n$ with probability at least $1-n^{-2}$. By applying the union bound over all $v \in V$, we obtain that a.a.s. $\left|N_{\mathcal{F}_{D}}(v)\right| \geq \delta n$ for all $v \in V$, as desired.

Finally, assume that $\delta n \leq|X| \leq\left(1-d^{k+2} 2^{-2 k-8}\right) n$. Here it suffices to show that, for any $X \subseteq V$ with $|X|=\delta n$, we have $\left|\bigcup_{v \in X} N_{\mathcal{F}_{D}}(v)\right| \geq\left(1-d^{k+2} 2^{-2 k-8}\right) n$. We use a similar argument as above, but this time we need to give more precise estimates. Consider any step $s$ of the algorithm, assume that $H_{s} \notin A$, and denote by $\mathcal{M}$ the history of the algorithm until this point. We want to bound the number of valid choices for $H_{s+1}$ in $\mathcal{H}^{\prime}$ that are neighbours of some $v \in X$ in $\mathcal{T}_{G}(\mathcal{H}, V)$. With Claim 7.1, there are at least $m_{0}-2 \varepsilon^{\prime} m_{0}-\varepsilon^{\prime} n-s$ choices for $H_{s+1} \in \mathcal{H}^{\prime}$ such that $H_{s} H_{s+1}$ is an edge of $F^{*}$ and $H_{s+1}$ is not a "dead-end". Using Claim 7.2, for at least $m_{0}-s-4 \varepsilon^{\prime} n$ of these choices, $H_{s+1}$ has degree at least $\left(1-4 \varepsilon^{\prime}\right) m_{0}$ in $F_{X}^{*}$. Then there are at least $m_{0}-s-4 \varepsilon^{\prime} m_{0}-\varepsilon^{\prime} n \geq m_{0}-s-5 \varepsilon^{\prime} n$ choices for $H_{s+2}$, such that $\left(H_{s+1}, H_{s+2}\right)$ is in $\bigcup_{v \in X} \mathcal{F}_{v}$.

On the other hand, there are at most $\left(m_{0}-s\right)$ choices for each of $H_{s+1}$ and $H_{s+2}$ and as above we have that at least one neighbour of $H_{s}$ or $H_{s+1}$ appears with probability at 
least $1-2 \varepsilon^{\prime}$. Using that $m_{0}-s \geq \delta n / 3$ we get

$$
\mathbb{P}\left[\left(H_{s+1}, H_{s+2}\right) \in \bigcup_{v \in X} \mathcal{F}_{v} \mid \mathcal{M} \wedge H_{s} \notin A\right] \geq \frac{\left(1-2 \varepsilon^{\prime}\right)^{2}\left(m_{0}-s-5 \varepsilon^{\prime} n\right)^{2}}{\left(m_{0}-s\right)^{2}} \geq 1-20 \frac{\varepsilon^{\prime}}{\delta} .
$$

Again, as the lower bound holds independently of the history $\mathcal{M}$ and as there are at most $\varepsilon^{\prime} n$ steps with $H_{s} \in A$, this process dominates a binomial distribution with parameters $t-\varepsilon^{\prime} n$ and $1-20 \frac{\varepsilon^{\prime}}{\delta}$. Therefore, the number $Y$ of these edges that are in $\bigcup_{v \in X} \mathcal{F}_{v}$ is in expectation at least $\left(t-\varepsilon^{\prime} n\right)\left(1-20 \frac{\varepsilon^{\prime}}{\delta}\right) \geq(1-\delta) t$ and we get from the more precise version of Chernoff's inequality (Lemma 2.1) that

$$
\begin{array}{r}
\mathbb{P}\left[Y<(1-2 \delta) t \leq \mathbb{E}[Y]-\left(\delta-20 \frac{\varepsilon^{\prime}}{\delta}\right)\left(t-\varepsilon^{\prime} n\right)\right] \\
\leq \exp \left(-D\left((1-\delta) \| 1-20 \frac{\varepsilon^{\prime}}{\delta}\right)\left(t-\varepsilon^{\prime} n\right)\right) \\
\leq \exp \left(-\delta\left(\log \left(\frac{\delta^{2}}{20 \varepsilon^{\prime}}\right)-2\right)\left(t-\varepsilon^{\prime} n\right)\right) \\
\leq \exp \left(-\delta \log \left(\frac{1}{\varepsilon^{\prime}}\right) \frac{1}{2} t\right) .
\end{array}
$$

There are at most $\left(\begin{array}{c}n \\ \delta n\end{array}\right) \leq\left(\frac{e}{\delta}\right)^{\delta n} \leq \exp \left(\delta \log \left(\frac{1}{\delta}\right) n\right) \leq \exp \left(\delta \log \left(\frac{1}{\varepsilon^{\prime}}\right) \frac{1}{4} t\right)$ choices for $X$ and, thus, with the union bound over all these choices, we obtain that a.a.s. at least $(1-2 \delta) t$ of the edges are in $\bigcup_{v \in X} \mathcal{F}_{v}$ for every $X \subseteq V$ with $|X|=\delta n$. At most $\varepsilon^{\prime} n$ of these edges do not belong to $D$ and putting this together we a.a.s. have

$$
\left|\bigcup_{x \in X} N_{\mathcal{F}_{D}}(x)\right| \geq(1-2 \delta) t-\varepsilon^{\prime} n \geq(1-4 \delta) n \geq\left(1-d^{k+2} 2^{-2 k-8}\right) n
$$

for any $X \subseteq V$ with $|X|=\delta n$, as wanted.

Let $D$ be the directed path in $F^{*}$ given by Claim 7.3 and assume that the assertion of Claim 7.4 holds. We denote the first vertex of $D$ by $H_{x}^{\prime}$ and the last by $H_{y}^{\prime}$. Before dealing with the next step, we summarise what we have so far. We have several copies of $H^{(k)}: H_{x}, H_{y}$ and those in $\mathcal{H}$. The vertices $x$ and $x^{\prime}$ (resp. $y$ and $y^{\prime}$ ) are adjacent in $G$ to all vertices of $H_{x}$ (resp. $H_{y}$ ), and thus $\left(x, x^{\prime}\right)$ and $\left(y, y^{\prime}\right)$ can be end-tuples of the square of a Hamilton path we want to construct. Moreover, we have an ordering (given by the directed path $D$ ) of $t$ copies of $H^{(k)}$ in $\mathcal{H}$, such that if $H=u_{1}, \ldots, u_{k}$ and $H=u_{1}^{\prime}, \ldots, u_{k}^{\prime}$ appear consecutively, then $u_{k} u_{1}^{\prime}$ is an edge of the random graph, and all their vertices $u_{1}, \ldots, u_{k}, u_{1}^{\prime}, \ldots, u_{k}^{\prime}$ have many common neighbours in $V$ in the graph $G$.

Covering the left-over vertices from $U_{1} \cup \cdots \cup U_{k}$. At this point, we still have $s=|\mathcal{H} \backslash V(D)|=|\mathcal{H}|-t=\frac{k}{3 k-1} \delta n$ copies of $H^{(k)}$ in $\mathcal{H}$ not used for the path $D$, and we let $\mathcal{H}^{\prime}$ be any subset of such copies of size $s-2$. Moreover, the number of vertices in $U_{i}$ not in any copy of $H^{(k)}$ in $\mathcal{H}$ is $\left|U_{i}\right|-2-|\mathcal{H}|=\frac{1}{3 k-1} \delta n=\frac{s}{k}$, and therefore we have exactly $s$ vertices in total in $U_{1} \cup \cdots \cup U_{k}$ to absorb; let $Z$ be the set of these vertices. We want to cover the $s$ vertices in $Z$ with the square of two paths connecting $H_{x}$ to $H_{x}^{\prime}$ and $H_{y}^{\prime}$ to $H_{y}$ respectively, while using all edges of $\mathcal{H}$ and exactly $4 s$ vertices from $V$ (c.f. Figure 8).

We start from connecting $H_{x}$ to $H_{x}^{\prime}$, while absorbing two vertices of $Z$. We pick $H^{\prime} \in \mathcal{H}^{\prime}$ and $z_{x}, z_{x}^{\prime} \in Z$ such that the vertices in $H_{x} \cup\left\{z_{x}\right\} \cup H^{\prime}$ and $H^{\prime} \cup\left\{z_{x}^{\prime}\right\} \cup H_{x}^{\prime}$ each have at least $2 \delta n$ common neighbours in $V$. This is possible by using Claim 7.1 and the regularity property of $G$. Then by Lemma 2.2 , a.a.s. the random graph $G_{2}$ has a path on four vertices within each of these two sets of $2 \delta n$ vertices, that gives the desired connection (c.f. Figure 8). 
Now we connect $H_{y}^{\prime}$ to $H_{y}$, while absorbing the other $s-2$ vertices of $Z \backslash\left\{z_{x}, z_{x}^{\prime}\right\}$. Let $H_{1}^{\prime}=H_{y}, H_{s-1}^{\prime}=H_{y}^{\prime}$ and $H_{2}^{\prime}, \ldots, H_{s-2}^{\prime}$ be a labelling of the remaining edges in $\mathcal{H}^{\prime} \backslash\left\{H^{\prime}\right\}$ such that for $j=1, \ldots, s-2$ we have that all vertices in $V\left(H_{j}\right) \cup V\left(H_{j+1}\right)$ have at least $d^{2 k+2} 2^{-2 k-7} n$ common neighbours in $V$ in $G$. This is possible by Dirac's Theorem and because, by Claim 7.1, for each $H \in \mathcal{H}^{\prime} \cup\left\{H_{y}, H_{y}^{\prime}\right\}$ all but $6 \frac{\varepsilon^{\prime}}{\delta} s$ choices $H^{\prime} \in \mathcal{H}^{\prime}$ are such that the vertices $V(H) \cup V\left(H^{\prime}\right)$ have $d^{2 k+2} 2^{-2 k-7} n$ common neighbours.

Next, we want to find a labelling $z_{1}, \ldots, z_{s-2}$ of the vertices from $Z^{\prime}=Z \backslash\left\{z_{x}, z_{x}^{\prime}\right\}$ such that for $j=1, \ldots, s-2$ the vertices $V\left(H_{j}\right) \cup\left\{z_{j}\right\} \cup V\left(H_{j+1}\right)$ have at least $2 \delta n$ common neighbours in $V$. This again follows easily from Hall's condition for perfect matchings and because, by Claim 7.1, for each $j=1, \ldots, s-2$ all but $6 \varepsilon^{\prime} s / \delta$ choices $z \in Z$ are such that $V\left(H_{j}\right) \cup V\left(H_{j+1}\right) \cup\{z\}$ have $2 \delta n$ common neighbours and, similarly, vice versa. Then by Lemma 2.2, a.a.s. we can greedily choose a path on four vertices in the common neighbourhood of the vertices from $V\left(H_{j}\right) \cup V\left(H_{j+1}\right) \cup\left\{z_{j}\right\}$ in $V$ for $j=1, \ldots, s-2$, with all the edges coming from the random graph $G_{2}$. This again gives the desired connection (c.f. Figure 8).

This completes the square of two paths from $H_{x}$ to $H_{x}^{\prime}$ and from $H_{y}^{\prime}$ to $H_{y}$. These two cover exactly $4 s$ vertices of $V$. Therefore, there are precisely $|V|-4-4 s=\left(1-\frac{4 k}{3 k-1} \delta\right) n=t$ vertices of $V \backslash\left\{x, x^{\prime}, y, y^{\prime}\right\}$ not yet covered by the square of a path, and we let $V^{\prime}$ be the set of such vertices and observe that $\left|V^{\prime}\right|=t=|V(D)|$.

Finishing the square of a path. We finish the proof by constructing the square of a path with $H_{x}^{\prime}$ and $H_{y}^{\prime}$ at the ends using precisely the vertices of $V^{\prime}$ and the copies of $H^{(k)}$ that are vertices of $V(D)$. For this we use that by Claim 7.4 there is a perfect matching in $\mathcal{F}_{D}\left[V^{\prime}, E(D)\right]$. For $i=1, \ldots, t$, let $v_{i}$ be the vertex of $V$ matched to the edge $\left(H_{i}, H_{i+1}\right) \in E(D)$ in $\mathcal{F}_{D}$. With $H_{i}=u_{1}, \ldots, u_{k}$ and $H_{i+1}=u_{1}^{\prime}, \ldots, u_{k}^{\prime}$, we then have that $v_{i}$ is incident to $u_{k-1} u_{k}, u_{1}^{\prime}, u_{2}^{\prime}$ by definition of $\mathcal{F}_{D}$. This completes the construction of the square of the path with $H_{x}^{\prime}$ to $H_{y}^{\prime}$ at the ends. By adding the two connections found above from $H_{x}$ to $H_{x}^{\prime}$ and from $H_{y}^{\prime}$ to $H_{y}$ and the initial tuples $\left(x, x^{\prime}\right)$ and $\left(y, y^{\prime}\right)$, we get the square of a Hamilton path with end-tuples $\left(x, x^{\prime}\right)$ and $\left(y, y^{\prime}\right)$ as desired (c.f. Figure 6). This finishes the proof of the lemma.

We end this section by giving the proof of Lemma 3.8, that follows from Lemma 3.7, once we split appropriately the super-regular regular pair $(U, V)$ into two copies of superregular $K_{1,2}$, both suitable for an application of Lemma 3.7 with $k=2$.

Proof of Lemma 3.8. Let $0<d<1$, choose $\delta^{\prime}$ with $0<\delta^{\prime} \leq \frac{1}{8} d$ and apply Lemma 3.7 with $k=2, \delta^{\prime}$, and $\frac{1}{8} d$ to obtain $\delta_{0}, \delta, \varepsilon^{\prime}$ with $\delta^{\prime} \geq \delta_{0}>\delta>\varepsilon^{\prime}>0$ and $C^{\prime}>0$. Then let $0<\varepsilon \leq \frac{1}{8} \varepsilon^{\prime}, C \geq 4 C^{\prime}$, and $p \geq C n^{-1}$. Next let $U$ and $V$ be vertex-sets of size $|V|=n$ and $3 n / 4 \leq|U|=m \leq n$ and assume that $(U, V)$ is a $(\varepsilon, d)$-super-regular pair. Let $\left(x, x^{\prime}\right)$ and $\left(y, y^{\prime}\right)$ be tuples from $V$ and $U$, respectively, such that they have $\frac{1}{2} d^{2} n$ common neighbours into the other set. We will reveal $G(V, p)$ and $G(U, p)$ both in two rounds as $G_{1}, G_{3} \sim G\left(V, \frac{1}{2} p\right)$, and $G_{2}, G_{4} \sim G\left(U, \frac{1}{2} p\right)$.

We partition $V$ into $V_{1}, U_{2}, W_{2}$ and $U$ into $V_{2}, U_{1}, W_{1}$ such that for $i=1,2$ the pairs $\left(U_{i}, V_{i}\right)$ and $\left(W_{i}, V_{i}\right)$ are $\left(\varepsilon^{\prime}, \frac{1}{8} d\right)$-super-regular pairs and $\left(1-\delta_{0}\right)\left|V_{i}\right| \leq\left|U_{i}\right|=\left|W_{i}\right| \leq(1-\delta)\left|V_{i}\right|$. Additionally, we require that $\left(x, x^{\prime}\right)$ is in $V_{1}$ and that $\left(y, y^{\prime}\right)$ is in $V_{2}$ and that they have at least $\frac{1}{2}\left(\frac{d}{8}\right)^{2} n$ common neighbours in $U_{1}, W_{1}$ and in $U_{2}, W_{2}$, respectively. To obtain this we split the sets according to the following random distribution. We put any vertex of $V$ into each of $U_{2}$ and $W_{2}$ with probability $q_{1}$ and into $V_{1}$ with probability $1-2 q_{1}$. Similarly, we put any vertex of $U$ into each of $U_{1}$ and $W_{1}$ with probability $q_{2}$ and into $V_{2}$ with probability $1-2 q_{2}$. We choose $q_{1}$ and $q_{2}$ such that the expected sizes satisfy for 
$i=1,2$

$$
\mathbb{E}\left[\left|U_{i}\right|\right]=\mathbb{E}\left[\left|W_{i}\right|\right]=\left(1-\frac{\delta_{0}+\delta}{2}\right) \mathbb{E}\left[\left|V_{i}\right|\right]
$$

This is possible since such conditions give a linear system of two equations in two unknowns $q_{1}$ and $q_{2}$, and, as $3 n / 4 \leq m \leq n$, the solution satisfies $1 / 7 \leq q_{1}, q_{2} \leq 3 / 7$. Then by Chernoff's inequality (Lemma 2.1) and with $n$ large enough there exists a partition such that for $i=1,2$ we have that $\left|W_{i}\right|,\left|U_{i}\right|$, and $\left|V_{i}\right|$ are all within $\pm n^{2 / 3}$ of their expectation and the minimum degree within both pairs $\left(U_{i}, V_{i}\right)$ and $\left(W_{i}, V_{i}\right)$ is at least a $d / 4$-fraction of the other set. For $i=1,2$ we redistribute $o(n)$ vertices between $U_{i}$ and $W_{i}$ and move at most one vertex from or to $V_{i}$ to obtain

$$
\left(1-\delta_{0}\right)\left|V_{i}\right| \leq\left|U_{i}\right|=\left|W_{i}\right| \leq(1-\delta)\left|V_{i}\right|
$$

with minimum degree within both pairs $\left(U_{i}, V_{i}\right)$ and $\left(W_{i}, V_{i}\right)$ at least a $d / 8$-fraction of the other set. Moreover, for $i=1,2$, we can ensure that with $n_{i}=\left|V_{i}\right|-4$ we have $n_{i}-\left|U_{i}\right| \equiv 0$ $(\bmod 10)$.

From this we get that for $i=1,2$ the pairs $\left(U_{i}, V_{i}\right)$ and $\left(W_{i}, V_{i}\right)$ are $\left(\varepsilon^{\prime}, \frac{1}{8} d\right)$-superregular. With $G_{1}$ and $G_{2}$ we reveal random edges within $V_{1}$ and $V_{2}$ with probability $p / 2$ to find tuples $\left(z, z^{\prime}\right)$ in $V_{1}$ and $\left(w, w^{\prime}\right)$ in $V_{2}$ such that together they give a copy of $K_{4}$ and $\left(z, z^{\prime}\right)$ and $\left(w, w^{\prime}\right)$ have at least $\frac{1}{2}\left(\frac{d}{8}\right)^{2} n$ common neighbours in $U_{1}, W_{1}$ and in $U_{2}$, $W_{2}$, respectively. Then we use Lemma 3.7 and $G_{3}, G_{4}$ with $C n^{-1} \geq C^{\prime} \min \left\{\left|V_{1}\right|,\left|V_{2}\right|\right\}^{-1}$ to a.a.s. find the square of a Hamilton path on $V_{i}, U_{i}, W_{i}$ for $i=1,2$ with end-tuples $\left(x, x^{\prime}\right),\left(z, z^{\prime}\right)$ and $\left(y, y^{\prime}\right),\left(w, w^{\prime}\right)$, respectively. Together with the edges between $\left(z, z^{\prime}\right)$ and $\left(w, w^{\prime}\right)$ this gives the square of a Hamilton path covering $U$ and $V$ with end-tuples $\left(x, x^{\prime}\right)$ and $\left(y, y^{\prime}\right)$.

\section{Concluding Remarks}

We determined the exact perturbed threshold for the containment of the square of a Hamilton cycle in randomly perturbed graphs. As already pointed out in Section 1.1, much less is known for larger powers of Hamilton cycles and it would be interesting to investigate them further. In this section we discuss the perturbed threshold $\hat{p}_{\alpha}(n)$ for the containment of the third power of a Hamilton cycle.

Recall from Section 1.1 that $\hat{p}_{\alpha}(n)$ is already known for $\alpha=0$ and $1 / 2<\alpha \leq 1$ : we have $\hat{p}_{\alpha}(n)=n^{-1 / 3}$ for $\alpha=0, \hat{p}_{\alpha}(n)=n^{-1}$ for $1 / 2<\alpha<3 / 4$, and $\hat{p}_{\alpha}(n)=0$ for $\alpha \geq 3 / 4$. For $0<\alpha<1 / 2$, it is only known that there exists $\eta>0$ such that $\hat{p}_{\alpha}(n) \leq n^{-1 / 3-\eta}$.

We observe that we can obtain natural lower bounds by determining the sparsest possible structure that remains for $G(n, p)$ after mapping the third power of a Hamilton cycle into the complete bipartite graph $H_{\alpha}$ with parts of size $\alpha n$ and $(1-\alpha) n$. When $\alpha=1 / 4$, this structure is essentially the square of a Hamilton cycle on $3 n / 4$, and is obtained by mapping every fourth vertex of the third power of a Hamilton cycle into the smaller part of $H_{1 / 4}$. Therefore, in order for $H_{1 / 4} \cup G(n, p)$ to contain the third power of a Hamilton cycle, we need $G(n, p)$ to contain the square of a Hamilton cycle on $3 n / 4$ vertices. This gives $\hat{p}_{1 / 4}(n) \geq n^{-1 / 2}$ and we believe this is actually tight.

Conjecture 8.1. The perturbed threshold $\hat{p}_{\alpha}(n)$ for the containment of the third power of a Hamilton cycle satisfies $\hat{p}_{1 / 4}(n)=n^{-1 / 2}$.

However, as discussed in the introduction, finding the square of a Hamilton cycle at this probability is a particularly challenging problem, and, additionally, it is not possible to first embed small parts arbitrarily and then connect them, as we do in the proof of our main result. 
For each of the ranges $0<\alpha<1 / 4$ and $1 / 4<\alpha<1 / 2$, it is not clear whether to expect a similar 'jumping' behaviour as the one proved for the square of a Hamilton cycle in the range $0<\alpha<1 / 2$. We can obtain natural lower bounds similarly as we did for $\alpha=1 / 4$. Again, the sparsest structure that remains for $G(n, p)$ is obtained essentially by mapping every $1 / \alpha$-th vertex of $C_{n}^{3}$ into the smaller part of $H_{\alpha}$. However, in contrast to Section 3.2, the threshold for the appearance of this structure in $G(n, p)$ is not determined by the second or third power of a short path. For example, when $\alpha=1 / 5$, by doing as described above and mapping every fifth vertex of $C_{n}^{3}$ into the smaller part of $H_{\alpha}$, we are left with copies of $P_{4}^{3}$, which are connected by three edges, cyclically. By a first moment argument, the threshold for this structure in $G(n, p)$ is at least $n^{-4 / 9}$, and thus $\hat{p}_{1 / 5}(n) \geq n^{-4 / 9}$, which is larger than the threshold for a $P_{4}^{3}$-factor in $G(n, p)$. More generally, we get for $0 \leq \alpha \leq 1 / 4$ that $\hat{p}_{\alpha}(n) \geq n^{-(1-\alpha) /(3-6 \alpha)}$, and for $1 / 4 \leq \alpha \leq 1 / 2$ that $\hat{p}_{\alpha}(n) \geq n^{-(1-\alpha) /(5 / 2-4 \alpha)}$. Note that these lower bounds match the already known value of $\hat{p}_{\alpha}(n)$ for $\alpha=0$ and coincide for $\alpha=1 / 4$, in support of Conjecture 8.1. Moreover, if it is tight, this 'continuous' perturbed threshold would be an exciting new behaviour.

Question 8.2. Does the perturbed threshold $\hat{p}_{\alpha}(n)$ for the containment of the third power of a Hamilton cycle satisfy

$$
\hat{p}_{\alpha}(n)= \begin{cases}n^{-(1-\alpha) /(3-6 \alpha)} & \text { if } \alpha \in\left[0, \frac{1}{4}\right), \\ n^{-(1-\alpha) /(5 / 2-4 \alpha)} & \text { if } \alpha \in\left[\frac{1}{4}, \frac{1}{2}\right] ?\end{cases}
$$

For neither of the two ranges of $\alpha$ this lower bound seems to be attainable with our approach, because at this probability there is no small structure that we can find and then connect into the third power of a Hamilton cycle. Taking again the example of $\alpha=1 / 5$, our lower bound for the perturbed threshold is at $n^{-4 / 9}$, but at this probability it is not possible to first find the copies of $P_{4}^{3}$ 's arbitrarily and then connect them. However we believe our methods can give the following. We map every ninth and tenth vertex of $C_{n}^{3}$ into the smaller part of $H_{1 / 5}$, and this leaves copies of $P_{8}^{3}$ connected by single edges, cyclically. We expect that it is possible to extend our argument to this set-up, but this would only imply $\hat{p}_{1 / 5}(n) \leq n^{-7 / 18}$. More generally, we believe we can show that $\hat{p}_{\alpha}(n) \leq n^{-(2 k-1) /(6 k-6)}$ for $\alpha \in\left(\frac{1}{k+1}, \frac{1}{k}\right)$ and $k \geq 4$, which is the threshold for the containment of linearly many copies of $P_{2 k}^{3}$ in $G(n, p)$. Similarly, we believe we can show that $\hat{p}_{\alpha}(n) \leq n^{-(3 k-1) /(6 k-3)}$ for $\alpha \in\left(\frac{k+1}{4 k+1}, \frac{k}{4 k-3}\right)$ and $k \geq 1$, which is the threshold for the containment of linearly many copies of $P_{3 k}^{2}$ in $G(n, p)$. This would improve on the bounds obtained in [6], but it is still far from the lower bounds discussed above. Note that the latter bound tends to $n^{-1 / 2}$, as $k$ tends to infinity (and thus $\alpha$ tends to $\frac{1}{4}$ ), supporting again Conjecture 8.1.

\section{REFERENCES}

1. M. Aigner and A. Brandt, Embedding arbitrary graphs of maximum degree two, J. London Math. Soc. (2) 48 (1993), no. 1, 39-51.

2. D. Angluin and L. G. Valiant, Fast probabilistic algorithms for Hamiltonian circuits and matchings, J. Comput. System Sci. 18 (1979), no. 2, 155-193.

3. S. Antoniuk, A. Dudek, C. Reiher, A. Ruciński, and M. Schacht, High powers of Hamiltonian cycles in randomly augmented graphs, J. Graph Theory 98 (2021), no. 2, 255-284.

4. J. Balogh, F. Mousset, and J. Skokan, Stability for vertex cycle covers, Electron. J. Combin. 24 (2017), no. 3, 25, P3.56.

5. T. Bohman, A. Frieze, and R. Martin, How many random edges make a dense graph Hamiltonian?, Random Structures Algorithms 22 (2003), no. 1, 33-42.

6. J. Böttcher, R. Montgomery, O. Parczyk, and Y. Person, Embedding spanning bounded degree graphs in randomly perturbed graphs, Mathematika 66 (2020), no. 2, 422-447. 
7. J. Böttcher, O. Parczyk, A. Sgueglia, and J. Skokan, Triangles in randomly perturbed graphs, arXiv:2011.07612, 2020.

8. _ Cycle factors in randomly perturbed graphs, arXiv:2103.06136, 2021.

9. __ Cycle factors in randomly perturbed graphs, Procedia Computer Science 195 (2021), 404411, Proceedings of the XI Latin and American Algorithms, Graphs and Optimization Symposium.

10. J. Böttcher, M. Schacht, and A. Taraz, Spanning 3-colourable subgraphs of small bandwidth in dense graphs, J. Combin. Theory Ser. B 98 (2008), no. 4, 752-777.

11. G. A. Dirac, Some theorems on abstract graphs, Proc. London Math. Soc. 2 (1952), 69-81.

12. A. Dudek, C. Reiher, A. Ruciński, and M. Schacht, Powers of Hamiltonian cycles in randomly augmented graphs, Random Structures Algorithms 56 (2020), no. 1, 122-141.

13. P. Erdős, Problem 9, Theory of Graphs and its Applications (Proc. Sympos. Smolenice, 1963) (M. Fiedler, ed.), Publ. House Czechoslovak Acad. Sci., Prague, 1964, p. 159.

14. A. Ferber, G. Kronenberg, and K. Luh, Optimal threshold for a random graph to be 2-universal, Trans. Amer. Math. Soc. 372 (2019), no. 6, 4239-4262.

15. M. Fischer, N. Škorić, A. Steger, and M. Trujić, Triangle resilience of the square of a Hamilton cycle in random graphs, J. Combin. Theory Ser. B 152 (2022), 171-220.

16. K. Frankston, J. Kahn, B. Narayanan, and J. Park, Thresholds versus fractional expectationthresholds, Ann. Math. (2) 194 (2021), no. 2, 475-495.

17. J. Han, P. Morris, and A. Treglown, Tilings in randomly perturbed graphs: bridging the gap between Hajnal-Szemerédi and Johansson-Kahn-Vu, Random Structures Algorithms 58 (2021), no. 3, 480516.

18. W. Hoeffding, Probability inequalities for sums of bounded random variables, J. Amer. Statist. Assoc. 58 (1963), 13-30.

19. S. Janson, T. Łuczak, and A. Ruciński, Random graphs, Wiley-Interscience Series in Discrete Mathematics and Optimization, Wiley-Interscience, New York, 2000.

20. A. Johansson, J. Kahn, and V. Vu, Factors in random graphs, Random Structures Algorithms 33 (2008), no. 1, 1-28.

21. J. Kahn, B. Narayanan, and J. Park, The threshold for the square of a Hamilton cycle, Proc. Amer. Math. Soc. 149 (2021), 3201-3208.

22. J. Komlós, G. N. Sárközy, and E. Szemerédi, On the square of a Hamiltonian cycle in dense graphs, Random Structures Algorithms 9 (1996), no. 1-2, 193-211.

23. J. Komlós, G. N. Sárközy, and E. Szemerédi, Proof of the Seymour conjecture for large graphs, Ann. Comb. 2 (1998), no. 1, 43-60.

24. J. Komlós and M. Simonovits, Szemerédi's regularity lemma and its applications in graph theory, Combinatorics, Paul Erdős is eighty, Vol. 2 (Keszthely, 1993), János Bolyai Math. Soc., Budapest, 1996, pp. 295-352.

25. D. Kühn and D. Osthus, On Pósa's conjecture for random graphs, SIAM J. Discrete Math. 26 (2012), no. $3,1440-1457$.

26. R. Nenadov and M. Trujić, Sprinkling a few random edges doubles the power, SIAM J. Discrete Math. 35 (2021), no. 2, 988-1004.

27. R. Nenadov and N. Škorić, Powers of Hamilton cycles in random graphs and tight Hamilton cycles in random hypergraphs, Random Structures Algorithms 54 (2019), no. 1, 187-208.

28. O. Parczyk, 2-universality in randomly perturbed graphs, European J. Combin. 87 (2020), 103-118.

29. L. Pósa, Hamiltonian circuits in random graphs, Discrete Math. 14 (1976), no. 4, 359-364.

30. O. Riordan, Spanning subgraphs of random graphs, Combin. Probab. Comput. 9 (2000), no. 2, 125148.

31. P. Seymour, Problem section, Combinatorics (Proc. British Combinatorial Conf. 1973) (T. P. McDonough and V. C. Mavron, eds.), Cambridge University Press, London-New York, 1974, pp. 201204. London Math. Soc. Lecture Note Ser., No. 13.

32. D. A. Spielman and S. Teng, Smoothed analysis of algorithms: why the simplex algorithm usually takes polynomial time, J. ACM 51 (2004), no. 3, 385-463.

33. E. Szemerédi, Regular partitions of graphs, Problèmes combinatoires et théorie des graphes (Colloques Internationaux CNRS, Univ. Orsay, Orsay, 1976), Colloq. Internat. CNRS, vol. 260, CNRS, Paris, 1978, pp. 399-401.

34. V. H. Vu, A large deviation result on the number of small subgraphs of a random graph, Combin. Probab. Comput. 10 (2001), no. 1, 79-94. 


\section{Appendix A. Supplementary PRoOfs}

In this section we will prove Lemmas 2.4, 2.9, 6.6, and 6.7. The proof of Lemma 2.9 generalises the argument in [7, Theorem 2.4], and the proof of Lemma 6.6 only requires basic regularity type arguments. The remaining two lemmas concern random graphs and their proofs are based on Janson's inequality (see e.g. [19, Theorem 2.18]).

Lemma A.1 (Janson's inequality). Let $p \in(0,1)$ and consider a family $\left\{H_{i}\right\}_{i \in \mathcal{I}}$ of subgraphs of the complete graph on the vertex set $[n]=\{1, \ldots, n\}$. For each $i \in \mathcal{I}$, let $X_{i}$ denote the indicator random variable for the event that $H_{i} \subseteq G(n, p)$ and, write $H_{i} \sim H_{j}$ for each ordered pair $(i, j) \in \mathcal{I} \times \mathcal{I}$ with $i \neq j$ if $E\left(H_{i}\right) \cap E\left(H_{j}\right) \neq \varnothing$. Then, for $X=\sum_{i \in \mathcal{I}} X_{i}$, $\mathbb{E}[X]=\sum_{i \in \mathcal{I}} p^{e\left(H_{i}\right)}$,

$$
\Delta[X]=\sum_{H_{i} \sim H_{j}} \mathbb{E}\left[X_{i} X_{j}\right]=\sum_{H_{i} \sim H_{j}} p^{e\left(H_{i}\right)+e\left(H_{j}\right)-e\left(H_{i} \cap H_{j}\right)}
$$

and any $0<\gamma<1$ we have

$$
\mathbb{P}[X \leq(1-\gamma) \mathbb{E}[X]] \leq \exp \left(-\frac{\gamma^{2} \mathbb{E}[X]^{2}}{2(\mathbb{E}[X]+\Delta[X])}\right) .
$$

Proof of Lemma 2.4. Let $s \geq 1$ and $k \geq 2$ be integers and $0<\eta \leq 1$. Moreover let $C \geq 2^{6}(s k)^{2 s k} \eta^{-2}$ and $p \geq C n^{-(k-1) /(2 k-3)}$.

Let $V$ be a vertex set of size $n, V_{i}$ be a subset of $V$ for $i=1, \ldots, s$, and $H$ be a collection of tuples from $\prod_{i=1}^{s} V_{i}^{k}$. Let $W_{i} \subseteq V_{i}$ for each $i=1, \ldots, s$ and assume $H^{\prime}=H \cap \prod_{i=1}^{s} W_{i}^{k}$ has size at least $\eta n^{s k}$. Since the number of tuples from $\prod_{i=1}^{s} V_{i}^{k}$ which contain a vertex more than once is $O\left(n^{s k-1}\right)$, there are at least $\frac{\eta}{2} n^{s k}$ tuples of $H^{\prime}$ such that their vertices are pairwise distinct. We restrict our analysis to the set of those tuples, which, abusing notation, we still denote by $H^{\prime}$.

For each tuple $\left(v_{i, j}: 1 \leq i \leq s, 1 \leq j \leq k\right)$ in $H^{\prime}$, we consider the graph with vertex set $V$ and the following edges. For $i=1, \ldots, s$ we have the square of the path on $v_{i, 1}, \ldots, v_{i, k}$ and for $i=1, \ldots, s-1$ we have the edge $v_{i, k} v_{i+1,1}$. This gives a family $\left\{H_{i}\right\}_{i \in\left[\left|H^{\prime}\right|\right]}$ of graphs with vertex set $V$ and, using the same notation as in Lemma A.1, a collection of random variables $\left\{X_{i}\right\}_{i \in\left[\left|H^{\prime}\right|\right]}$. Note that for each $i=1, \ldots, s$, we have $e\left(H_{i}\right)=s(2 k-3)+(s-1)=$ $2 s(k-1)-1$, and thus, for $X=\sum_{i \in\left[\left|H^{\prime}\right|\right]} X_{i}$, we have $\mathbb{E}[X]=\left|H^{\prime}\right| p^{2 s(k-1)-1} \geq \sqrt{C} n$. To compute the quantity $\Delta[X]=\sum_{H_{i} \sim H_{j}} p^{e\left(H_{i}\right)+e\left(H_{j}\right)-e\left(H_{i} \cap H_{j}\right)}$, we split the sum according to the number of vertices in the intersection $E\left(H_{i} \cap H_{j}\right)$. Suppose $H_{i}$ and $H_{j}$ intersect in $m$ vertices. Then $2 \leq m \leq s k-1$ and the largest size $\tilde{e}(m)$ of the intersection $E\left(H_{i} \cap H_{j}\right)$ is

$$
\tilde{e}(m)= \begin{cases}\frac{m}{k}(2 k-3)+\frac{m}{k}-1, & \text { if } m \equiv 0 \quad(\bmod k) \\ \left\lfloor\frac{m}{k}\right\rfloor(2 k-3)+\left\lfloor\frac{m}{k}\right\rfloor, & \text { if } m \equiv 1 \quad(\bmod k) \\ \left\lfloor\frac{m}{k}\right\rfloor(2 k-3)+\left\lfloor\frac{m}{k}\right\rfloor+2\left(m-k\left\lfloor\frac{m}{k}\right\rfloor\right)-3, & \text { otherwise. }\end{cases}
$$

In particular, observing that $\tilde{e}(m)=2 m-3$ if $m<k$ (as $m \geq 2$ we are in the third case) and $\tilde{e}(m) \leq 2 m-2 \frac{m}{k}-1$ if $m \geq k$ (the inequality follows from $\left\lfloor\frac{m}{k}\right\rfloor \geq \frac{m}{k}-1$ ), we can conclude that $p^{-\tilde{e}(m)} n^{-m} \leq C^{-1} n^{-1}$ for each $2 \leq m \leq s k-1$. Therefore,

$$
\begin{aligned}
\Delta[X] & \leq \sum_{m=2}^{s k-1} m !\left(\begin{array}{c}
s k \\
m
\end{array}\right)^{2} n^{2 s k-m} p^{[2 s(k-1)-1]+[2 s(k-1)-1]-\tilde{e}(m)} \leq \sum_{m=2}^{s k-1}(s k)^{2 m} \frac{n^{2 s k-m}}{\left|H^{\prime}\right|^{2}} \mathbb{E}^{2}[X] p^{-\tilde{e}(m)} \\
& \leq 4(s k)^{2 s k-2} \eta^{-2} \sum_{m=2}^{s k-1} \mathbb{E}^{2}[X] p^{-\tilde{e}(m)} n^{-m} \leq 4(s k)^{2 s k-1} \eta^{-2} C^{-1} \mathbb{E}^{2}[X] n^{-1} \leq \frac{1}{8} s^{-1} \mathbb{E}^{2}[X] n^{-1},
\end{aligned}
$$


where in the first inequality we used that there are at most $m !\left(\begin{array}{c}s k \\ m\end{array}\right)^{2} n^{2 s k-m}$ choices for $H_{i}$ and $H_{j}$ intersecting in $m$ vertices, in the second we used $\mathbb{E}[X]=\left|H^{\prime}\right| p^{2 s(k-1)-1} \geq \sqrt{C} n$, and in the third we used $n^{s k} /\left|H^{\prime}\right| \leq 2 / \eta$. Then with Lemma A.1 applied with $\gamma=2^{-1 / 2}$, we get that the probability that none of the graphs of the family $\left\{H_{i}\right\}_{i \in\left[\left|H^{\prime}\right|\right]}$ appears in $G(n, p)$ is bounded from above by

$$
\begin{aligned}
\exp \left(-\frac{\mathbb{E}^{2}[X]}{4(\mathbb{E}[X]+\Delta[X])}\right) & \leq \exp \left(-\frac{1}{8} \min \left\{\mathbb{E}[X], \mathbb{E}^{2}[X] / \Delta[X]\right\}\right) \\
& \leq \exp \left(-\frac{1}{8} \min \{\sqrt{C} n, 8 s n\}\right) \leq \exp (-s n)
\end{aligned}
$$

We can conclude with a union bound over the at most $2^{s n}$ choices for the $s$ subsets $W_{i}$ with $i=1, \ldots, s$ that the lemma holds.

Proof of Lemma 2.9. Let $k \geq 2$ and $t \geq 1$ be integers. For $k=2$ the result follows from the proof of [7, Theorem 2.4] with slight modifications, so we can assume $k \geq 3$. We let $0<\gamma<(16 k t)^{-1}$ and $C \geq 2^{k+9} k t$. Further let $p \geq C(\log n)^{1 /(2 k-3)} n^{-(k-1) /(2 k-3)}, 1 \leq m \leq \gamma n$, and let $G$ be an $n$-vertex graph with vertex set $V$, minimum degree $\delta(G) \geq m$ and maximum degree $\Delta(G) \leq \gamma n$.

We distinguish two cases. If $m \leq(\log n)^{2 /(2 k-3)} n^{(2 k-4) /(2 k-3)}$, we only need Janson's inequality and we will greedily find $t m+t$ copies of $P_{k+1}^{2}$, using only edges from the random graph $G(n, p)$. Let $V^{\prime} \subseteq V$ be the set of vertices used in this greedy construction. As long as we have not found $t m+t$ copies of $P_{k+1}^{2}$, we have $\left|V^{\prime}\right| \leq(t m+t)(k+1)$ and thus $\left|V \backslash V^{\prime}\right| \geq n / 2$. We let $\left\{H_{i}\right\}_{i \in \mathcal{I}}$ be the family of copies of $P_{k+1}^{2}$ with vertices in $V \backslash V^{\prime}$ and note $|\mathcal{I}| \geq 2^{-k-2} n^{k+1}$. Then, using the notation of Lemma A.1, we observe that the expected number of these copies appearing as subgraphs of $G(n, p)$ is

$$
\mathbb{E}[X]=|\mathcal{I}| p^{2 k-1} \geq 2^{-k-2} n^{k+1} p^{2 k-1} \geq C(\log n)^{(2 k-1) /(2 k-3)} n^{(2 k-4) /(2 k-3)} \geq 32 k t m \log n .
$$

On the other hand, we have

$$
\begin{aligned}
\Delta[X] & =\sum_{H_{i} \sim H_{j}} p^{e\left(H_{i}\right)+e\left(H_{j}\right)-e\left(H_{i} \cap H_{j}\right)} \leq \sum_{r=2}^{k} O\left(n^{2(k+1)-r} p^{2(2 k-1)-(2 r-3)}\right) \\
& \leq \mathbb{E}^{2}[X] \sum_{r=2}^{k} O\left(p^{3-2 r} n^{-r}\right) \leq \mathbb{E}^{2}[X] o\left(n^{-1}\right)
\end{aligned}
$$

where in the first inequality we split the sum according to the value of $r=v\left(H_{i} \cap H_{j}\right)$ and used that then $e\left(H_{i} \cap H_{j}\right) \leq 2 r-3$. Then with Lemma A.1 we get that the probability that there is no copy of $P_{k+1}^{2}$ is bounded from above by $\exp (-\mathbb{E}[X] / 8) \leq n^{-4 k t m}$. We conclude with a union bound over the at most $\left(\begin{array}{c}n \\ (k+1)(t m+t)\end{array}\right) \leq n^{2 k t m}$ possible choices for $V^{\prime}$ that we can a.a.s. find $t m+t$ copies of $P_{k+1}^{2}$ in $G(n, p)$.

For $m \geq(\log n)^{2 /(2 k-3)} n^{(2 k-4) /(2 k-3)}$ we need to use the edges of $G$. We will find copies of $P_{k+1}^{2}$, where all edges incident to one vertex come from $G$ and the remaining edges come from $G(n, p)$, where we will need to distinguish between $k=3$ and $k \geq 4$. First, we greedily obtain a spanning bipartite subgraph $G^{\prime} \subseteq G$ of minimum degree $\delta\left(G^{\prime}\right) \geq m / 2$ by taking a partition of $V(G)$ into sets $A$ and $B$ such that $e_{G}(A, B)$ is maximised and letting $G^{\prime}=G[A, B]$. Indeed, a vertex of degree less than $m / 2$ can be moved to the other class to increase $e_{G}(A, B)$. W.l.o.g. we assume $|B| \geq n / 2 \geq|A|$. Moreover, we have $|A| \geq m /(4 \gamma)$, as otherwise with $e(A, B) \geq n \mathrm{~m} / 4$ there is a vertex of degree at least $\gamma n$, a contradiction.

Then we observe that given any sets $A^{\prime} \subseteq A$ and $B^{\prime} \subseteq B$ such that $\left|A^{\prime}\right| \leq m /(16 \gamma)$ and $\left|B^{\prime}\right| \leq n / 4$, we also have $e\left(A \backslash A^{\prime}, B \backslash B^{\prime}\right) \geq n m / 16$. Otherwise, from $e\left(A, B \backslash B^{\prime}\right) \geq \mid B \backslash$ $B^{\prime} \mid m / 2 \geq n m / 8$, it would follow that $e\left(A^{\prime}, B \backslash B^{\prime}\right) \geq n m / 16$ and thus, since $\left|A^{\prime}\right| \leq m /(16 \gamma)$, 
we would have a vertex of degree at least $\gamma n$ in $A^{\prime}$, a contradiction to the maximum degree of $G$.

We will greedily find $t m+t$ copies of $P_{k+1}^{2}$ with one vertex in $A$ and $k$ vertices in $B$. Let $A^{\prime} \subseteq A$ and $B^{\prime} \subseteq B$ be the set of vertices used in this greedy construction. As long as we have not found $t m+t$ copies of $P_{k+1}^{2}$, we have $\left|A^{\prime}\right| \leq t m+t \leq m /(16 \gamma)$ and $\left|B^{\prime}\right| \leq k(t m+t) \leq n / 4$, and thus $e\left(A \backslash A^{\prime}, B \backslash B^{\prime}\right) \geq n m / 16$. Therefore, using that $|A| \leq n / 2$, there is a vertex $v \in A \backslash A^{\prime}$ with degree at least $m / 8$ into $B \backslash B^{\prime}$. We let $B^{*}$ be a set of $m / 8$ neighbours of $v$ in $B \backslash B^{\prime}$.

When $k=3$, we will find a path on three vertices in $B^{*}$ in the random graph, which will give, together with the three edges of $G$ between $v$ and those vertices, a copy of $P_{4}^{2}$. We argue as follows. If such a path does not appear, then there are less than $m$ edges of $G(n, p)$ in $B^{*}$. However the expected number of random edges within $B^{*}$ is at least $p\left(\begin{array}{c}m / 8 \\ 2\end{array}\right) \geq 8 k t m \log n$, and therefore, by Lemma 2.1 , with probability at least $1-n^{-4 k t m}$ there are more than $m$ edges of $G(n, p)$ in $B^{*}$. We conclude by union bound over the at most

choices for $A^{\prime}$ and $B^{\prime}$.

$$
\left(\begin{array}{c}
|A| \\
t m+t
\end{array}\right)\left(\begin{array}{c}
|B| \\
k(t m+t)
\end{array}\right) \leq n^{2 k t m}
$$

For $k \geq 4$ we let $B_{1}, \ldots, B_{4}$ be pairwise disjoint sets of size $m / 32$ in $B^{*}$. Moreover, we let $B_{5}, \ldots, B_{k}$ be pairwise disjoint sets of size $n /(4 k)$ in $B \backslash B^{\prime}$, each disjoint from $B_{1}, \ldots, B_{4}$. This is possible as $\left|B \backslash B^{\prime}\right| \geq n / 4$.

Claim A.2. With probability at least $1-n^{-\omega(m)}$ there exists vertices $b_{1}, \ldots, b_{k}$ with $b_{i} \in B_{i}$ for $i=1, \ldots, k$ such that in $G(n, p)$ we have the edges $b_{i} b_{i+1}$ for $i=1, \ldots, k-1$ and $b_{i} b_{i+2}$ for $i=3, \ldots, k-2$.

Observe that, together with $v$ and the edges $v b_{i}$ for $i=1, \ldots, 4$, this gives a copy of $P_{k+1}^{2}$ with vertices $b_{1}, b_{2}, v, b_{3}, \ldots, v_{k}$. As there are at most $n^{O(m)}$ choices for $A^{\prime}$ and $B^{\prime}$, by a union bound and Claim A.2, we a.a.s. find $t m+t$ copies of $P_{k+1}^{2}$. It remains to prove the claim.

Proof of Claim A.2. We denote by $\left\{H_{i}\right\}_{i \in \mathcal{I}}$ the graphs on $k$ vertices $b_{1}, \ldots, b_{k}$ with $b_{i} \in B_{i}$ for $i=1, \ldots, k$ and edges $b_{i} b_{i+1}$ for $i=1, \ldots, k-1$ and $b_{i} b_{i+2}$ for $i=3, \ldots, k-2$. Then, using the notation of Lemma A.1, the expected number of those graphs appearing in $G(n, p)$ is

$$
\begin{aligned}
\mathbb{E}[X] & =|\mathcal{I}| p^{2 k-5} \geq \Omega\left(m^{4} n^{k-4} p^{2 k-5}\right) \\
& \geq \Omega\left(m(\log n)^{\frac{6}{2 k-3}+\frac{2 k-5}{2 k-3}} n^{(k-4)+\frac{3(2 k-4)}{2 k-3}-\frac{(2 k-5)(k-1)}{2 k-3}}\right)=\omega(m \log n),
\end{aligned}
$$

where we used the bounds on the sizes of the sets $B_{i}$ for $i=1, \ldots, k$ in the first inequality and the bounds on $m$ and $p$ in the second inequality. On the other hand we get

$$
\begin{aligned}
\Delta[X] & =\sum_{H_{i} \sim H_{j}} p^{e\left(H_{i}\right)+e\left(H_{j}\right)-e\left(H_{i} \cap H_{j}\right)} \leq \sum_{r, s} O\left(m^{8-r} n^{2 k-8-s} p^{2(2 k-5)-(2 s+\min \{2 r-3, r-1\})}\right) \\
& \leq \mathbb{E}^{2}[X] \sum_{r, s} O\left(m^{-r} n^{-s} p^{-2 s-\min \{2 r-3, r-1\}}\right) \leq \mathbb{E}^{2}[X] O\left(n^{-2} p^{-1}\right) \leq \mathbb{E}^{2}[X] o\left(n^{-1}\right),
\end{aligned}
$$

where we split the sum according to the value of $r$ and $s$, with $0 \leq r \leq 4,0 \leq s \leq k-4$, and $2 \leq r+s \leq k-1$, where $r$ and $s$ are the number of common vertices of $H_{i}$ and $H_{j}$ in $B_{1}, \ldots, B_{4}$ and $B_{5}, \ldots, B_{k}$, respectively. In the first inequality we used that $e\left(H_{i} \cap H_{j}\right) \leq$ $2 s+\min \{2 r-3, r-1\}$, and in the third inequality we used that $O\left(m^{-r} n^{-s} p^{-2 s-\min \{2 r-3, r-1\})}\right)$ is maximised for $r=0$ and $s=2$ with the given bounds on $m$ and $p$. The claim follows by Lemma A.1, as in the application above. 
Proof of Lemma 6.6. To prove (i), without loss of generality, it suffices to show that the degree of every vertex in $U_{1}$ is at least $(1-h \varepsilon) m^{h-1}$. Fix any $u_{1} \in U_{1}$ and set $N_{1}=$ $N_{G}\left(u_{1}, V\right)$. Notice that since $\left(V, U_{1}\right)$ is $(\varepsilon, d)$-super-regular, we have $\left|N_{1}\right| \geq d|V| \geq \varepsilon|V|$. Since $\left(V, U_{2}\right)$ is $(\varepsilon, d)$-super-regular, there are at least $(1-\varepsilon) m$ vertices $u_{2} \in U_{2}$ such that the set $N_{2}=N_{G}\left(u_{2}, N_{1}\right)$ of neighbours of $u_{2}$ in $N_{1}$ has size at least $(d-\varepsilon)\left|N_{1}\right| \geq$ $(d-\varepsilon) d|V| \geq \varepsilon|V|$. Continuing in the same way, by applying Lemma 2.6 to the $(\varepsilon, d)$ super-regular pair $\left(V, U_{j}\right)$ for $j=3, \ldots, h$, we get that there are at least $((1-\varepsilon) m)^{j-1}$ choices of $\left(u_{2}, \ldots, u_{h}\right) \in U_{2} \times \cdots \times U_{j}$ such that the vertices $u_{1}, u_{2}, \ldots, u_{j}$ have at least $(d-\varepsilon)^{j-1}\left|N_{1}\right| \geq(d-\varepsilon)^{j-1} d|V| \geq(d-\varepsilon)^{h-1} d|V| \geq \varepsilon|V|$ common neighbours in the set $V$. Since $(d-\varepsilon)^{h-1}\left|N_{1}\right| \geq \frac{1}{2} d^{h} n$ and $((1-\varepsilon) m)^{h-1} \geq(1-h \varepsilon) m^{h-1}$, the first part of the lemma follows.

Without loss of generality, it suffices to prove (ii) for $U_{1}$. If $|X| \geq 2 \varepsilon n d^{1-h}$, then, by applying Lemma 2.6, for all but at most $\varepsilon m$ vertices $u_{1} \in U_{1}$, the set $N_{1}=N\left(u_{1}, X\right)$ of neighbours of $u_{1}$ in $X$ is of size at least $(d-\varepsilon)|X|$. Fix any such $u_{1}$ and proceed in the same way as in the proof of (i). We get that there are at least $((1-\varepsilon) m)^{h-1} \geq(1-h \varepsilon) m^{h-1}$ choices of $\left(u_{2}, \ldots, u_{h}\right) \in U_{2} \times \cdots \times U_{h}$ such that the vertices $u_{1}, u_{2}, \ldots, u_{h}$ have at least $(d-\varepsilon)^{h-1}\left|N_{1}\right| \geq(d-\varepsilon)^{h}|X| \geq \frac{1}{2} d^{h}|X|$ common neighbours in the set $X$, and the second part of the lemma follows.

Proof of Lemma 6.7. Given any graph $H$ on $h \geq 2$ vertices and any $\delta>0$, we fix $\varepsilon>0$ with $\varepsilon<2^{-4 h-24} h^{-8} \delta^{4 h}$, and we let $\delta^{\prime}=2^{-3} h^{-1} \varepsilon^{1 / 4}$ and large enough for the inequalities indicated below to hold. Observe that the maximum degree of $F$ is $m^{h-1}$ and, by Lemma 6.6(i), the minimum degree of $F$ is at least $(1-h \varepsilon) m^{h-1}$. Therefore

$$
\mathbb{E}[e(\tilde{F})]=e(F) p^{e(H)}=(1 \pm h \varepsilon) m^{h} p^{e(H)}
$$

and

$$
\begin{aligned}
\operatorname{Var}[e(\tilde{F})] & =O_{h, \delta}\left(\sum_{H^{\prime} \subseteq H, e\left(H^{\prime}\right)>0} m^{2 v(H)-v\left(H^{\prime}\right)}\left(p^{2 e(H)-e\left(H^{\prime}\right)}-p^{2 e(H)}\right)\right) \\
& =O_{h, \delta}\left(\mathbb{E}[e(\tilde{F})]^{2} \sum_{H^{\prime} \subseteq H, e\left(H^{\prime}\right)>0} m^{-v\left(H^{\prime}\right)} p^{-e\left(H^{\prime}\right)}\right) \\
& =O_{h, \delta}\left(\mathbb{E}[e(\tilde{F})]^{2} \sum_{H^{\prime} \subseteq H, e\left(H^{\prime}\right)>0} n^{-v\left(H^{\prime}\right)} p^{-e\left(H^{\prime}\right)}\right)=O_{h, \delta}\left(\mathbb{E}[e(\tilde{F})]^{2} C^{-1} n^{-1}\right),
\end{aligned}
$$

where we used that $n^{-v\left(H^{\prime}\right)} p^{-e\left(H^{\prime}\right)} \leq C^{-e\left(H^{\prime}\right)} n^{-v\left(H^{\prime}\right)+e\left(H^{\prime}\right) / m_{1}(H)} \leq C^{-e\left(H^{\prime}\right)} n^{-1}$ in the last step. Using Chebyshev's inequality, we have

$$
\mathbb{P}[e(\tilde{F}) \neq(1 \pm \varepsilon) \mathbb{E}[e(\tilde{F})]]=O_{h, \delta, \varepsilon}\left(\frac{\operatorname{Var}[e(\tilde{F})]}{\mathbb{E}[e(\tilde{F})]^{2}}\right)=O_{h, \delta, \varepsilon}\left(C^{-1} n^{-1}\right),
$$

and thus a.a.s.

$$
e(\tilde{F})=(1 \pm \varepsilon) \mathbb{E}[e(\tilde{F})]=(1 \pm \varepsilon)(1 \pm h \varepsilon) m^{h} p^{e(H)} .
$$

Similarly as above, given $U_{i}^{\prime} \subseteq U_{i}^{\prime}$ of size at least $\delta m$ for $i=1, \ldots, h$, we have

$$
\mathbb{E}\left[e\left(\tilde{F}^{\prime}\right)\right]=e\left(F^{\prime}\right) p^{e(H)}=\left(1 \pm h \frac{\varepsilon}{\delta}\right) \prod_{i=1}^{h}\left|U_{i}^{\prime}\right| p^{e(H)}=\Omega_{h, \delta, \varepsilon}\left(n^{h} p^{e(H)}\right)=\Omega_{h, \delta, \varepsilon}(C n)
$$


and $\Delta\left[e\left(\tilde{F}^{\prime}\right)\right]=O_{h, \delta, \varepsilon}\left(\mathbb{E}\left[e\left(\tilde{F}^{\prime}\right)\right]^{2} C^{-1} n^{-1}\right)$. Then with Lemma A.1 we have

$$
\mathbb{P}\left[e\left(\tilde{F}^{\prime}\right)<(1-\varepsilon) \mathbb{E}\left[e\left(\tilde{F}^{\prime}\right)\right]\right] \leq \exp \left(-\frac{\varepsilon^{2} \mathbb{E}\left[e\left(\tilde{F}^{\prime}\right)\right]^{2}}{2 \Delta\left[e\left(\tilde{F}^{\prime}\right)\right]+2 \mathbb{E}\left[e\left(\tilde{F}^{\prime}\right)\right]}\right) \leq \exp (-h n),
$$

where the last inequality holds for large enough $C$, and we conclude with a union bound that a.a.s.

$$
e\left(\tilde{F}^{\prime}\right) \geq(1-\varepsilon) \mathbb{E}\left[e\left(\tilde{F}^{\prime}\right)\right] \geq(1-\varepsilon)\left(1-h \frac{\varepsilon}{\delta}\right) \prod_{i=1}^{h}\left|U_{i}^{\prime}\right| p^{e(H)} \geq(1-\sqrt{\varepsilon}) \prod_{i=1}^{h}\left|U_{i}^{\prime}\right| p^{e(H)}
$$

for all choices of $U_{i}^{\prime} \subseteq U_{i}^{\prime}$ of size at least $\delta m$ for $i=1, \ldots, h$ and using the choice of $\varepsilon$. This proves the lower bound of (5). Note that (7) and (8) hold also with $\delta$ replaces by $\delta^{\prime}$.

Next we upper bound $e\left(\tilde{F}^{\prime}\right)$ by taking $e(\tilde{F})$ and subtracting those edges of $\tilde{F}$ that are not in $\tilde{F}^{\prime}$, i.e. the edges that contain at least one vertex $v_{i}$ that belongs to $U \backslash U_{i}^{\prime}$. We only need a lower bound on their number and we will see that it is enough to lower bound those for which $\left|U_{i} \backslash U_{i}^{\prime}\right| \geq \delta^{\prime} m$ (which can be done using (8)), and simply ignore the others. For this we let $J \subseteq[h]$ be the set of those indices $j \in[h]$ such that $\left|U_{j}^{\prime}\right| \leq\left(1-\delta^{\prime}\right) m$, and for any $\varnothing \neq I \subseteq J$ we let $F_{I}$ be the subgraph of $F$ induced by the sets $U_{i} \backslash U_{i}^{\prime}$ for $i \in I$ and $U_{i}^{\prime}$ for $i \notin I$. Using (7) and (8), we get

$$
e\left(\tilde{F}^{\prime}\right) \leq e(\tilde{F})-\sum_{\varnothing \neq I \subseteq J} e\left(\tilde{F}_{I}\right) \leq(1+\varepsilon) \mathbb{E}[e(\tilde{F})]-\sum_{\varnothing \neq I \subseteq J}(1-\varepsilon) \mathbb{E}\left[e\left(\tilde{F}_{I}\right)\right],
$$

that we can further upper bound by

$$
\begin{aligned}
& (1+\varepsilon)(1+h \varepsilon) \prod_{i=1}^{h}\left|U_{i}\right| p^{e(H)}-\sum_{\varnothing \neq I \subseteq J}(1-\varepsilon)\left(1-h \frac{\varepsilon}{\delta^{\prime}}\right) \prod_{i \in I}\left|U_{i} \backslash U_{i}^{\prime}\right| \prod_{i \notin I}\left|U_{i}^{\prime}\right| p^{e(H)} \\
& \leq \prod_{j \notin J}\left|U_{j}\right| \prod_{j \in J}\left|U_{j}^{\prime}\right| p^{e(H)}+2 h \varepsilon \prod_{i=1}^{h}\left|U_{i}\right| p^{e(H)}+2 h \frac{\varepsilon}{\delta^{\prime}} \sum_{\varnothing \neq I \subseteq J} \prod_{i \in I}\left|U_{i} \backslash U_{i}^{\prime}\right| \prod_{i \notin I}\left|U_{i}^{\prime}\right| p^{e(H)} \\
& \leq\left(1-\delta^{\prime}\right)^{|J|-h} \prod_{i=1}^{h}\left|U_{i}^{\prime}\right| p^{e(H)}+2 h \varepsilon \delta^{-h} \prod_{i=1}^{h}\left|U_{i}^{\prime}\right| p^{e(H)}+2 h \frac{\varepsilon}{\delta^{\prime}} 2^{|J|} \delta^{-|J|} \prod_{i=1}^{h}\left|U_{i}^{\prime}\right| p^{e(H)} \\
& \quad \leq\left(1+2 h \delta^{\prime}+2 h \varepsilon \delta^{-h}+2^{h+1} h \delta^{-h} \frac{\varepsilon}{\delta^{\prime}}\right) \prod_{i=1}^{h}\left|U_{i}^{\prime}\right| p^{e(H)} \leq(1+\sqrt{\varepsilon}) \prod_{i=1}^{h}\left|U_{i}^{\prime}\right| p^{e(H)} .
\end{aligned}
$$

To get the second line, we used $(1+\varepsilon)(1+h \varepsilon) \leq 1+2 h \varepsilon,(1-\varepsilon)\left(1-h \frac{\varepsilon}{\delta^{\prime}}\right) \geq 1-2 h \frac{\varepsilon}{\delta^{\prime}}$ and

$$
\prod_{i=1}^{h}\left|U_{i}\right|-\sum_{\varnothing \neq I \subseteq J} \prod_{i \in I}\left|U_{i} \backslash U_{i}^{\prime}\right| \prod_{i \notin I}\left|U_{i}^{\prime}\right|=\prod_{j \notin J}\left|U_{j}\right| \prod_{j \in J}\left|U_{j}^{\prime}\right|
$$

as we are left with those edges that have vertices in $U_{j} \backslash U_{j}^{\prime}$ for $j \notin J$ and in $U_{j}^{\prime}$ for $j \in J$. To get to the third line, we used that for $j \notin J$ we have $\left|U_{j}^{\prime}\right| \geq\left(1-\delta^{\prime}\right) m=\left(1-\delta^{\prime}\right)\left|U_{j}\right|$ and that for each $i$ we have $\left|U_{i} \backslash U_{i}^{\prime}\right| \leq\left|U_{i}\right| \leq \delta^{-1}\left|U_{i}^{\prime}\right|$. In the last estimate we use the bound on $\varepsilon$ and the choice of $\delta^{\prime}$. This finishes the proof of (5).

For (6), we repeat essentially the same argument we used for the lower bound of (5). Observe that from (ii) of Lemma 6.6, if $|X| \geq 2 \varepsilon n d^{1-h}$, all but at most $\varepsilon m$ vertices from each $U_{i}$ have degree at least $(1-h \varepsilon) m^{h-1}$ in $F_{X}$. Therefore

$$
\mathbb{E}\left[e\left(\tilde{F}_{X}^{\prime}\right)\right]=e\left(F_{X}^{\prime}\right) p^{e(H)}=\left(1 \pm h \frac{\varepsilon}{\delta}\right) \prod_{i=1}^{h}\left|U_{i}^{\prime}\right| p^{e(H)}=\Omega_{h, \delta, \varepsilon}(C n)
$$


and again $\Delta\left[e\left(\tilde{F}_{X}^{\prime}\right)\right]=O_{h, \varepsilon, \delta}\left(\mathbb{E}\left[e\left(\tilde{F}_{X}^{\prime}\right)\right]^{2} C^{-1} n^{-1}\right)$. Then with Lemma A.1 we have

$$
\mathbb{P}\left[e\left(\tilde{F}_{X}^{\prime}\right)<(1-\varepsilon) \mathbb{E}\left[e\left(\tilde{F}_{X}^{\prime}\right)\right]\right] \leq \exp \left(-\frac{\varepsilon^{2} \mathbb{E}\left[e\left(\tilde{F}_{X}^{\prime}\right)\right]^{2}}{2 \Delta\left[e\left(\tilde{F}_{X}^{\prime}\right)\right]+2 \mathbb{E}\left[e\left(\tilde{F}_{X}^{\prime}\right)\right]}\right) \leq \exp (-h n),
$$

where the last inequality holds for large enough $C$. Then with a union bound over all choices of $U_{1}^{\prime}, \ldots, U_{h}^{\prime}$ we conclude that $e\left(\tilde{F}_{X}^{\prime}\right) \geq(1-\varepsilon) \mathbb{E}\left[e\left(\tilde{F}_{X}^{\prime}\right)\right]$ with probability $1-e^{-n}$, and using (9), we finish the proof. 